\title{
ScanNet: An interpretable geometric deep learning model for structure-based protein binding site prediction
}

Jérôme Tubiana ( $\square$ jertubiana@gmail.com )

Tel Aviv University

Dina Schneidman-Duhovny

Hebrew University of Jerusalem

Haim Wolfson

Tel Aviv University

Article

Keywords: ScanNet, deep learning, functional sites

Posted Date: September 21st, 2021

DOl: https://doi.org/10.21203/rs.3.rs-877980/v1

License: (1) This work is licensed under a Creative Commons Attribution 4.0 International License.

Read Full License

Version of Record: A version of this preprint was published at Nature Methods on May 30th, 2022. See the published version at https://doi.org/10.1038/s41592-022-01490-7. 


\title{
${ }_{1}$ ScanNet: An interpretable geometric deep learning model for structure-based protein binding site prediction

Jérôme Tubiana*

Blavatnik School of Computer Science, Tel Aviv University, Israel

Dina Schneidman-Duhovny ${ }^{\dagger}$

School of Computer Science and Engineering, The Hebrew University of Jerusalem, Israel

Haim J. Wolfson ${ }^{\ddagger}$

Blavatnik School of Computer Science, Tel Aviv University, Israel

(Dated: September 5, 2021)

\begin{abstract}
Predicting the functional sites of a protein from its structure, such as the binding sites of small molecules, other proteins or antibodies sheds light on its function in vivo. Currently, two classes of methods prevail: Machine Learning (ML) models built on top of handcrafted features and comparative modeling. They are respectively limited by the expressivity of the handcrafted features and the availability of similar proteins. Here, we introduce ScanNet, an end-to-end, interpretable geometric deep learning model that learns features directly from 3D structures. ScanNet builds representations of atoms and amino acids based on the spatio-chemical arrangement of their neighbors. We train ScanNet for detecting protein-protein and protein-antibody binding sites, demonstrate its accuracy - including for unseen protein folds - and interpret the filters learned. Finally, we predict epitopes of the SARS-CoV-2 spike protein, validating known antigenic regions and predicting previously uncharacterized ones. Overall, ScanNet is a versatile, powerful, and interpretable model suitable for functional site prediction tasks. A webserver for ScanNet is available from http://bioinfo3d.cs.tau.ac.il/ScanNet/
\end{abstract}

24 Despite recent progresses in experimental [1] and AI-based [2,3] protein structure determination, there remains 25 a gap between structure and function [4]. The most accurate functional site prediction method is comparative 26 modelling [5-13]: given a query protein, similar proteins with known functional sites are searched for and their sites ${ }_{27}$ are mapped onto the query structure. Comparative modelling has several shortcomings. First and foremost, its ${ }_{28}$ coverage is limited, as the pool of experimentally characterized protein folds or structural motifs is small. Second, ${ }_{29}$ functional sites are variably preserved throughout evolution. On the one hand, the B-cell epitopes of viral proteins 30 frequently undergo antigenic drift, i.e. the abolition of recognition by antibodies after only one or few mutations. ${ }_{31}$ On the other hand, some protein-protein interactions are mainly driven by few "hotspot" residues; mutations and/or 32 conformational changes of the other interface residues preserve the interaction. Put differently, the invariances in both ${ }_{33}$ sequence and conformation spaces of such function-determining structural motifs are in general motif-dependent and 34 therefore unknown. This hampers our ability to both define and recognize such motifs using conventional comparative 35 approaches.

An alternative to comparative modelling is feature-based Machine Learning (ML) [12-18]. For each amino acid ${ }_{37}$ of a query protein, various features of geometrical (e.g. secondary structure, solvent accessibility, molecular surface 38 curvature), physico-chemical (e.g. hydrophobicity, polarity, electrostatic potential) and evolutionary (e.g. conserva${ }_{39}$ tion, position- weight matrices, coevolution) nature are calculated. Then, the target property is predicted using a ML 40 model for tabular data such as Random Forest or Gradient Boosting. Reasoning on mathematically defined features ${ }_{41}$ offers three advantages: i) ability to generalize to proteins with no similarity to any of the train set proteins, ii) high ${ }_{42}$ sequence sensitivity, i.e. ability to output distinct predictions for highly similar protein sequences and iii) fast infer${ }_{43}$ ence speed. ML models are however limited by the expressiveness of the features employed, as these cannot capture 44 the spatio-chemical arrangements of atoms or amino acids characterizing function-bearing motifs. Examples of such 45 function-bearing motifs include Zinc fingers that are signature of DNA/RNA binding sites [19], or protein-protein 46 interaction hotspots "O-rings" [20], namely exposed hydrophobic/aromatic amino acids surrounded by polar/charged ${ }_{47}$ ones. Despite over 50 years of experimental structural determination, novel function-determining motifs are still being 48 discovered [21].

49 End-to-end differentiable models, i.e. Deep Learning (DL) can potentially overcome the limitations of both ap${ }_{50}$ proaches. Indeed, DL models can learn the data features and their invariances directly by backpropagation, and

\footnotetext{
* jertubiana@gmail.com

$\dagger$ dina.schneidman@mail.huji.ac.il

‡wolfson@tau.ac.il
} 
${ }_{51}$ generalize well despite large number of parameters. Adapting the DL approach to protein structures requires defining ${ }_{52}$ an appropriate representation for proteins. Proteins can indeed be represented in multiple, complementary ways, ${ }_{53}$ e.g. as sequences [22, 23], residue graphs [24-27], atomic density maps [28-34], atomic point clouds [35] or molecu${ }_{54}$ lar surfaces [36, 37], each capturing different functionally relevant features. Voxelated atomic density maps can be ${ }_{55}$ readily processed using classical 3D Convolutional Neural Networks, but the approach is computationally intensive 56 and the predictions are not invariant upon rotation of the input structure. Point clouds, graphs and surfaces can be ${ }_{57}$ analyzed via Geometric Deep Learning [38, 39], i.e. end-to-end differentiable models tailored for data with no natural ${ }_{58}$ grid-like topology or shared global coordinate system. Graphs can be derived from 3D structures by taking residues ${ }_{59}$ as nodes and the distances and angles between them as edges and processed using Graph Neural Networks (GNN) 60 such as Message Passing Neural Networks [40] or Graph Attention Networks [41]. By design, GNNs are invariant ${ }_{61}$ upon euclidean transformation and expressive, but can be challenging to regularize and interpret. In particular, it is 62 unclear whether - and if yes, which - structural motifs are captured by GNNs.

${ }_{63}$ Here, we introduce ScanNet (Spatio-Chemical Arrangement of Neighbors Neural Network), a novel geometric deep ${ }_{64}$ learning architecture tailored for protein structures. ScanNet builds representations of atoms and amino acids based ${ }_{65}$ on the spatio-chemical arrangement of their neighbors and exploits them to predict labels for each amino acid. By ${ }_{66}$ construction, ScanNet is end-to-end differentiable with minimal structure preprocessing, yielding fast training and ${ }_{67}$ inference. ScanNet predictions are local, invariant upon euclidean transformations and integrate information from ${ }_{68}$ multiple scales (atom, amino acid) and modalities (structure, MSA) in a synergistic fashion. Its corresponding ${ }_{69}$ parametric function is expressive, meaning that it can efficiently approximate known handcrafted features. Crucially, 70 through appropriate parameterization and regularization, the filters learnt by ScanNet can be readily visualized and 71 interpreted. We showcase the capabilities of ScanNet on two related tasks: prediction of protein-protein binding sites 72 and B-cell epitopes (i.e. antibody binding sites). ScanNet outperforms baseline methods based on ML, structural 73 homology and surface-based geometric deep learning. We further visualize and interpret the representations learnt 74 by the network. We find that they encompass known handcrafted features, and find filters detecting simple, generic 75 structural motifs such as hydrogen bonds as well as filters recognizing complex, task-specific motifs such as O-rings 76 and transmembrane helical domains. Applied to the SARS-CoV-2 spike protein, ScanNet predictions validate known 77 antigenic regions and predict a previously uncharacterized one.

\section{RESULTS}

\section{A. Spatio-chemical Arrangement of Neighbors Network (ScanNet)}

80 ScanNet takes as input a protein structure file, and optionally, a position-weight matrix derived from a multiple ${ }_{81}$ sequence alignment and outputs a residue-wise label probability. Its four main stages, shown in Fig. 1 and detailed in 82 Materials and Methods, are: atomic neighborhood embedding, atom to amino acid pooling, amino acid neighborhood 83 embedding and neighborhood attention.

${ }_{84}$ ScanNet first builds, for each heavy atom, a local coordinate frame centered on its position and oriented according 85 to its covalent bonds. Next, it identifies its closest neighboring atoms. The resulting neighborhood, formally a point ${ }_{86}$ cloud with coordinates and attributes (atom group type) is passed through a set of spatio-chemical linear filters ${ }_{87}$ to yield an atom-wise representation. Each filter outputs a matching score between its (trainable) spatio-chemical ${ }_{88}$ pattern and the neighborhood. The patterns, which are parameterized using Gaussian kernels and sparse bilinear 89 products, are localized in both physical and attribute space. Localization facilitates interpretation and is biologically 90 motivated since motif functionality is often born by a few key atomic groups / amino acids in a specific arrangement, 91 whereas other neighbors are irrelevant and interchangeable. Trainable, localized spatio-chemical patterns generalize ${ }_{92}$ to proteins the well-known concept of pharmacophores for small molecules.

${ }_{93}$ Towards calculation of amino acid-wise output, the atom-wise representation is pooled at the amino acid scale 94 and concatenated with embedded amino acid-level information (either amino acid type or position-weight matrix). ${ }_{95}$ Importantly, the constituting atoms of an amino acid have various types and may play different functional roles. In ${ }_{96}$ particular, some handcrafted features such as accessible surface area average information over all the atoms, whereas ${ }_{97}$ others, such as secondary structure consider only subsets (the backbone atoms). Therefore, a trainable, multi-headed ${ }_{98}$ attention pooling operation capable of learning which atoms are relevant for each feature is employed rather than a 99 conventional symmetric pooling operation like average or maximum.

${ }_{100}$ The neighborhood embedding procedure is then repeated at the amino acid scale: a local coordinate frame is 101 constructed for each amino acid from its $C_{\alpha}$ atom, side-chain orientation and local backbone orientation and its 102 nearest neighbors are identified. The resulting neighborhood with learnt attributes is passed through a set of trainable ${ }_{103}$ filters to yield an amino-acid wise representation.

${ }_{104}$ Finally, spatially-consistent output probabilities are obtained by projecting the amino-acid representations to scalar 
105 values, averaging them across a local neighborhood and converting to probabilities with a logistic function. The 106 averaging scheme integrates two specifics of protein binding sites. First, protein-protein interactions are frequently ${ }_{107}$ driven by key "hotspot" residues that contribute most of the binding energy, whereas other "passenger" nearby residues 108 have a small contribution to the binding energy [20, 42]. Such passenger residues are harder to detect directly as 109 they do not necessarily have the salient features of protein-protein binding sites [43]. Second, some amino acid pairs 110 consistently have opposite binding site labels - in particular, consecutive amino acids along the sequence because 111 their side chains typically point in opposite directions. Altogether, this motivates the introduction of trainable, 112 attention-based weighted averages, with algebraic weights.

\section{B. ScanNet for prediction of protein-protein binding sites}

114 The protein-protein Binding Sites (PPBS) of a protein are defined as the residues directly involved in one or 115 more native, high affinity protein-protein interaction (PPI). Not every surface residue is a PPBS, as (i) binding ${ }_{116}$ propensity competes with structural stability and (ii) PPI are highly partner and conformation-specific. Knowledge ${ }_{117}$ of the PPBS of a protein provides insight about its in-vivo behavior, particularly when its partners are unknown and 118 can guide docking algorithms. Prediction of PPBS with conventional approaches is challenging as PPBS structural 119 motifs are more diverse, less conserved and more extended than small molecule binding sites. Additionally, only 120 incomplete and noisy labels can be derived from structural data, as (i) most PPIs of a given protein are not structurally ${ }_{121}$ characterized, and (ii) a substantial fraction $(\sim 15 \%$ [44]) of the structurally characterized protein-protein interfaces 122 are not physiological but crystal-induced.

${ }_{123}$ We constructed a non-redundant data set of 20K representative protein chains with annotated binding sites derived 124 from the Dockground database of protein complexes [45]. The PPBS data set covers a wide range of complex sizes, 125 types, organism taxonomies, protein lengths (Fig.S4 (a)-(d)) and contains around 5M amino acids, of which 22.7\% 126 are PPBS. To address the uneven sampling of the protein space, we introduced sample weights for each chain that ${ }_{127}$ are inversely proportional to the number of similar chains found in the data set (Materials and Methods and Sup. ${ }_{128}$ Fig. S4(h)). To investigate the relationship between homology and generalization error, we divided the validation/test ${ }_{229}$ sets into four splits based on the degree of homology with respect to their closest train set example (see Fig. 2 and ${ }_{130}$ Sup. Fig. S4(g)).

${ }_{131}$ We evaluated three models on the PPBS data set: (i) ScanNet, (ii) a ML pipeline based on handcrafted features 132 and (iii) a structural homology pipeline (see Materials and Methods for technical details). For the handcrafted ${ }_{133}$ features baseline, we computed for each amino acid various geometric, chemical and evolutionary features, and used 134 xgboost, a state-of-the-art tree-based classification algorithm [46]. For the structural homology pipeline, pairwise ${ }_{135}$ local structural alignments between the train set chains and the query chain were first constructed using MultiProt 136 [47]. Then, alignments were weighted and aggregated to produce binding site probabilities for each amino acid. For 137 all three models, the validation set was used for hyperparameters selection and early stopping, and performance is 138 reported on the test set. Training and evaluation of a single model took one to two hours for ScanNet (excluding 139 preprocessing time, $\sim 10 \mathrm{~ms}$ per step using a single Nvidia V100 GPU), few minutes for the ML baseline (excluding 140 feature calculation time, using Intel Xeon Phi processor with 28 cores) and one month for the structural homology ${ }_{141}$ baseline (Intel Xeon Phi processor with 28 cores). We also evaluated Masif-site [36], a surface-based geometric deep 142 learning model. Since Masif-site was not trained on the same data set, we only report its global test set performance.

${ }_{143}$ We found that for the full test set, ScanNet achieved an AUCPR of 0.694 (Table I), accuracy of 87.7\% (Sup. Table ${ }_{144} \mathrm{~S} 3$ ) and $73.5 \%$ precision at $50 \%$ recall (Sup. Fig. S14), the best performance by a substantial margin. The next 145 best model was the structural homology baseline, whereas Masif-site and the handcrafted features model performed 146 similarly. The model ranks differed when considering only subsets (Fig. 2 (a)-(d)). Unsurprinsingly, the structural ${ }_{147}$ homology baseline performed best in the high homology setting, but its performance degraded rapidly with the degree 148 of relatedness; when the test protein had no similar fold in the train set, it was the worst algorithm. Conversely, the 149 performance of the handcrafted features baseline increased slowly with the degree of homology, meaning that it could 150 not faithfully memorize previously seen folds. In contrast, ScanNet could both memorize previously seen folds and 151 generalize to unseen ones.

${ }_{152}$ Visualizations of ScanNet predictions for representative examples (Fig. 2 (e),(f) and Sup. Fig. S7,S8,S9,S10) ${ }_{153}$ illustrate that predictions are spatially coherent and that in most cases, the binding sites are correctly identified. ${ }_{154}$ Overall, the network performed uniformly well across complex types and sizes, protein lengths and organisms (Sup. ${ }_{155}$ Fig.S6 ). PPBS identification was slightly harder when no or few homologs were found in the MSA (Sup. Fig.S6 b) 156 and slightly easier for enzymes (Sup. Fig.S6 d). We next identified and visualized train and test examples on which ${ }_{157}$ ScanNet performed poorly (Sup. Fig. S11). We found bona fide false negative (undetected interacting patches) and ${ }_{158}$ false positives (predicted interacting patches), although for the later we could not rule out involvement in another ${ }_{159}$ PPI for which no structural data was available. Another source of mistake was confusion between types of binding 
160 sites: we found at least one instance where the incorrectly predicted PPBS were actually RNA binding sites. Finally, ${ }_{161}$ confusion between crystal and native interfaces was a substantial source of apparent mistakes. We found several 162 train set examples in which the network "refused" to learn the train label and instead predicted another binding 163 interface with high confidence (Sup. Fig. S12). The predicted binding sites matched well the interface found in 164 another biological assembly file. We found a posteriori that the biological assembly files used in the train set were 165 annotated as probably incorrect by QSbio [44]. Overall, this demonstrated the robustness of predictions with respect 166 to noise in training labels.

${ }_{167}$ We next performed ablation experiments to investigate the importance of the network components (Table I, Sup. ${ }_{168}$ Fig. S13, Sup. Fig. S14). ScanNet performance decreased but remained above the other methods when discarding the 169 evolutionary information (by replacing the position-weight matrix by the one-hot encoded sequence) or all the atomic170 scale information (by removing the first two modules). Removing the sparse regularization on the spatio-chemical ${ }_{171}$ patterns and the early stopping yielded an homology-like performance profile, with better performance in the high 172 homology setting but poorer otherwise. Lastly, training the model on all chains without redundancy reduction nor 173 using sample weights yielded worse performance, highlighting the importance of sample weights.

${ }_{174}$ Finally, we investigated the impact of conformational changes upon binding (i.e. induced fit) on ScanNet predictions 175 using the Dockground unbound X-ray and simulated data sets [45]. Overall, predictions based on bound and unbound 176 structures were highly consistent, and accuracy decreased only mildly from bound to unbound (Materials and Methods 177 Sec. D, Sup. Fig. S5, Sup. Table S1).

\begin{tabular}{|c|c|c|c|c|c|}
\hline Algorithm & Test (70\%) & Test (Homology) & Test (Topology) & Test (None) & Test (All) \\
\hline Structural homology baseline & $\mathbf{0 . 8 2 8}$ & 0.696 & 0.535 & 0.387 & 0.613 \\
Handcrafted features baseline & 0.596 & 0.567 & 0.568 & 0.432 & 0.537 \\
Masif-site [36] & NA & NA & NA & NA & 0.533 \\
ScanNet & 0.733 & $\mathbf{0 . 7 1 2}$ & $\mathbf{0 . 7 3 5}$ & $\mathbf{0 . 6 0 5}$ & $\mathbf{0 . 6 9 4}$ \\
\hline ScanNet (no evolutionary information) & 0.672 & 0.648 & 0.685 & 0.565 & 0.639 \\
ScanNet (no atomic information) & 0.697 & 0.672 & 0.689 & 0.547 & 0.648 \\
ScanNet (no regularization) & 0.756 & 0.702 & 0.701 & 0.572 & 0.678 \\
ScanNet (no reweighting) & 0.702 & 0.668 & 0.683 & 0.553 & 0.648 \\
\hline
\end{tabular}

TABLE I. Performance evaluation for prediction of Protein-protein binding sites. Area under Precision Recall Curve (AUCPR) is shown. Proteins of the test set are subdivided into four non-overlapping groups. Test 70\%: At least $70 \%$ sequence identity with at least one train set example. Test Homology: At most $70 \%$ sequence identity with any train set example, at least one train set example belonging to same protein superfamily (H level of CATH classification [48]). Test Topology: At least one train set example with similar protein topology (T level of CATH classification [48]), none with similar protein superfamily. Test None: None of the above. For Masif-site, only the aggregated performance is shown since its training set differs from ours. See Sup. Tables S2,S3 for additional evaluation metrics

\section{Visualization and interpretation of the learnt representations}

179 What did ScanNet learn? Does the network reason solely by comparison with training instances or does it learn 180 the underlying chemical principles of binding? How will it behave in out-of-sample settings such as AlphaFold models ${ }_{181}[2]$ or disordered regions? To better understand the learnt representations, we visualized the spatio-chemical patterns 182 and low-dimensional projections of the representations at the atomic (Fig. 3) and amino acid (Fig. 4) levels.

183 Recall that each pattern is composed by a set of gaussian kernels characterized by their location in the local ${ }_{184}$ coordinate system and specificity in attribute space. At the atomic scale, the origin corresponds to the central 185 atom and the z-axis and xz-plane are oriented according to its covalent bonds. Panels (a)-(f) of Fig. 3,4 each show 186 one pattern (left), together with a maximally activating neighborhood (right) taken from the validation set and the ${ }_{187}$ remaining patterns are provided as Supplementary Data. The atomic pattern shown in Fig. 3 (a) has two main ${ }_{188}$ components: a $N H$ group located at the center and an oxygen located few $\AA$ away, in the $(x<0, y<0, z<0)$ 189 quadrant, i.e. opposite from the two covalent bonds. It is the well known signature of a $N-H-O$ hydrogen 190 bond, ubiquitous in protein backbones. The corresponding maximally activating atom is indeed a backbone nitrogen 191 within a beta sheet. Patterns may have more than two components, and several possible groups per location. The 192 atomic pattern shown in panel (b) features two oxygen atoms and three $N H$ groups in a specific arrangement; the ${ }_{193}$ corresponding maximally activating neighborhoods are backbone nitrogens located at contact zones between two 194 helical fragments (right of panel (b) and Sup Fig.S15). Patterns shown in panels (c),(d) focus on side chains. Pattern 195 (c) is defined as a carbon in the vicinity of a methyl group and an aromatic ring. Pattern (d) consists of $S H$ or ${ }_{196} \mathrm{NH}_{2}$ groups - two side chains-located hydrogen donors - surrounded by oxygen atoms. Lastly, patterns may include ${ }_{197}$ prescribed absence of atoms in specific regions. Pattern (e) is defined by a backbone carbon or oxygen without any 
${ }_{198} N H$ groups in its vicinity, meaning that it identifies backbones available for hydrogen bonding. Pattern (f) identifies 199 a methionine side chain with one solvent-exposed side, and is associated with high PPBS probability. Together, the 200 filters collectively define a rich representation capturing various properties of a neighborhood, as seen from the 2D T${ }_{201}$ SNE projections colored by properties (Fig. $3(\mathrm{~g}),(\mathrm{h})$ ). In the space of filter activities, atoms cluster by coordination 202 number (number of other atoms in range of Van Der Waals interaction) and electrostatic potential (calculated with 203 the Adaptive Poisson-Boltzmann Solver [49]).

204 The amino acid scale patterns can be similarly analyzed: the origin, $z$ axis and $x z$ plane are respectively defined 205 by the $C_{\alpha}$, side-chain and backbone orientation of the central amino acid. Neighborhoods are shown as backbone 206 segments, with position weight matrices as attributes; the learnt attributes pooled from the atomic scale are not ${ }_{207}$ shown. Each gaussian component of a pattern is characterized by a complex specificity in attribute space. We 208 represent it by the distributions of amino acid types and accessible surface areas of its top 1\% maximally activating 209 residues. Patterns (a) and (b) focus only on the central amino acid, i.e. they recombine and propagate features from 210 the previous layers. Pattern a) consists of solvent exposed residues of type frequently encountered in protein-protein 211 interfaces such as Leucine or Arginine. It is positively correlated with the output probability $(r=0.31)$. Conversely, 212 pattern (b), which consists of buried hydrophobic amino acids, is activated by residues within the protein cores and 213 is negatively correlated with the output $(r=-0.32)$.

${ }_{214}$ Multi-component patterns are also found: pattern (c) consists of an exposed glycine together with and exposed 215 aromatic or leucine amino acid and is correlated with binding $(r=0.18)$. Pattern $(\mathrm{d})$ is constituted by an exposed 216 hydrophobic amino acid surrounded by exposed, charged amino acids and is strongly correlated with binding $(r=$ 217 0.29). It is remarkably similar to the hotspot O-ring architecture previously described by Bogan and Thorn [20]. ${ }_{218}$ Conversely, pattern (e), which consists of a central cysteine (possibly involved in a disulfide bond) surrounded by 219 exposed lysines is negatively correlated with binding $(r=-0.13)$.

220 Distributed patterns such as pattern (f) are found and hypothetically contribute to prediction by identifying domain221 level context. Pattern (f), which consists of multiple aromatic and hydrophobic components, is strongly activated by 222 transmembrane helical domains. Identification of transmembrane domain is indeed required for accurate prediction ${ }_{223}$ as the hydrophobic core / hydrophilic rim rule is reversed within membranes. Finally, the two dimensional T-SNE ${ }_{224}$ projections of the representation (Fig. 4 (f), (g) and Sup. Fig. S16) show that the filter activities encompass various 225 amino-acid level handcrafted features, including amino acid type, secondary structure, accessible surface area, surface 226 convexity and evolutionary conservation.

\section{ScanNet for prediction of B-cell Epitopes}

${ }_{228}$ B-cell epitopes (BCE) are defined as residues directly involved in a antibody-antigen complex. Although a priori 229 every surface residue is potentially immunogenic, some are preferred in the sense that it is easier to mature antibodies 230 targeting them with high affinity and specificity. Exhaustive, high-throughput experimental determination of BCEs 231 is challenging, because they can span across multiple non-contiguous protein fragments. Prediction is challenging 232 owing to their instability throughout evolution, and the lack of exhaustive epitope mappings for a given antigen. In${ }_{233}$ silico prediction of $\mathrm{BCE}$ can be leveraged for constructing epitope-based vaccines and for designing non-immunogenic 234 therapeutic proteins.

235 We derived from the SabDab database [50] a data set of 3756 protein chains (796 95\% sequence identity clusters) 236 with annotated BCE. $8.9 \%$ of the residues were labeled as BCE, likely an underestimation of the true fraction. The ${ }_{237}$ data set was split into five subsets for cross-validation training, with no more than $70 \%$ sequence identity between ${ }_{238}$ pairs of sequences from different subsets. We evaluated ScanNet in three settings: trained from scratch, trained for ${ }_{239}$ PPBS prediction without finetuning, and trained via transfer learning using the PPBS network as starting point. We 240 compared it with the handcrafted features baseline, structural homology baseline and Discotope, a popular tool based ${ }_{241}$ on geometric features and propensity scores [51]. We also report the performance of ScanNet without evolutionary 242 data, of the null predictor and of a predictor based on solvent accessibility only. ScanNet trained via transfer learning ${ }_{243}$ outperformed the other models, with an AUCPR of 0.178 and a Positive Predicted Value at L/10 of 27.5\% (Fig. 5 244 (a), Sup. Table S4). This represents an enrichment of respectively 143\%, 153\% and 309\% over Discotope, solvent 245 accessibility-based and null prediction. ScanNet perfomed equally well with or without evolutionary information 246 unlike for PPBS. Visualization of representative spatio-chemical patterns associated with high BCE probability sheds 247 light on the similarities and differences between PPBS and BCE (Fig. 5 (b)-(e), the remaining filters are provided ${ }_{248}$ as Supplementary Data). We find Asparagine and Arginine-containing patterns (b,c) as well as linear epitopes ( 249 (c), shared with PPBS). Pattern (d) consists of exposed residues with alternate charges, and putatively indicates 250 availability for salt-bridge formation (d). Finally, pattern (e) is constituted by an exposed, charged amino acid in the 251 vicinity of two cysteines forming a disulfide bond. A possible explanation is that disulfide bond-rich regions are more ${ }_{252}$ structurally stable, hence easier to recognize with high affinity and specificity. 
${ }_{253}$ We next predicted and visualized BCE of the SARS-CoV-2 spike protein. Predictions are shown with representative 254 antibodies superimposed for the trimer with one open Receptor Binding Domain (RBD) (Fig. 5 (e)) and for the isolated ${ }_{255} \mathrm{RBD}$ and N-terminal domain (NTD) (Sup. Fig. S17). For the spike protein, the RBD was correctly identified as a 256 major antigenic site. The six main epitopes previously described [52] all had high probabilities, including the cryptic ${ }_{257}$ epitope CR3022 (exposed in the open conformation). The tip of the N-terminal Domain (NTD) was also correctly ${ }_{258}$ identified as a highly antigenic site. Two linear epitopes located in the S2 fusion machinery are also predicted around ${ }_{259}$ Glu 1150 and Arg 1185 respectively. Previously, Shrock et al. [53] reported that both regions were targeted by 260 antibodies from recovered Covid-19 patients. For the first one, a broadly neutralizing mAB targeting this epitope was ${ }_{261}$ recently isolated [54] and shown to neutralize several beta-coronaviruses but not SARS-CoV-2. Finally, the network 262 predicted with high confidence one previously unreported conformational epitope constituted by three fragments in ${ }_{263}$ the vicinity of the glycosylated [55] Asn 657. Since the presence of the glycosyl group is unknown at run-time but can 264 be imputed by ScanNet from the Asn-X-Ser/Thr linear motif, two interpretations are possible: either the glycosyl ${ }_{265}$ group shields an otherwise highly immunogenic region from antibodies or it directly induces immune response via ${ }_{266}$ glycosyl-binding antibodies. We similarly found two additional cryptic epitopes of the NTD which are centered on 267 glycosylated asparagine when performing prediction on the NTD domain alone (Sup. Fig. S17 (b) ).

${ }_{268}$ Overall, ScanNet predictions are in excellent agreement with the known antigenic profile of the spike protein 269 and predict a novel epitope that could not be detected via high-throughput linear epitope scanning. We additionally ${ }_{270}$ predicted BCE for three other viral protein: HIV envelope protein, influenza HA-1 and influenza HA-3 Hemagglutinin ${ }_{271}$ (Sup. Fig. S18). We notably found that the Hemagglutinin epitope predictions differed between the HA-1 and HA-3 272 strand despite the similar fold, suggesting that ScanNet could be suitable for studying antigenic drift.

${ }_{274}$ Protein function is born by a diverse set of structural motifs. These motifs, characterized by their complex spatio275 chemical arrangements of atoms and amino acids, cannot be fully encompassed by handcrafted features. Conversely, 276 detection via comparative modeling is challenging because their invariants, i.e. the set of function-preserving se277 quence/conformational perturbations are unknown. ScanNet is an end-to-end geometric deep learning model capable 278 of learning such motifs together with their invariants directly from raw structural data by backpropagation. We 279 demonstrated, through a detailed comparison on newly compiled datasets of annotated protein-protein binding sites 280 and B-cell epitopes that it efficiently leverages these motifs to outperform feature-based methods, comparative mod281 eling, and surface-based geometric deep learning. ScanNet reaches an accuracy of $87.7 \%$ for PPBS prediction and a ${ }_{282}$ positive prediction value at $\mathrm{L} / 10$ of $27.5 \%$ for BCE prediction. Through appropriate parameterization and regular283 ization, the spatio-chemical patterns learned by the model can be explicitly visualized and interpreted as previously 284 known motifs and as novel ones.

${ }_{285}$ A breakthrough was recently achieved in protein structure prediction using DL [2], leading to the release of a vast ${ }_{286}$ set of accurate protein structure models [3]. We anticipate that ScanNet will prove insightful for analyzing these ${ }_{287}$ proteins, of which little is known regarding their function. A webserver is made available at http://bioinfo3d. ${ }_{288}$ cs.tau.ac.il/ScanNet/ and linked to both the PDB and AlphaFoldDB for ease of use. Owing to its generality, 289 it is straightforward to extend the model to other classes of binding sites provided that sufficient training data is 290 available. Extension to partner-specific binding prediction for prediction interactions and guiding molecular docking 291 is a promising future direction. A second class of applications is protein design: ScanNet, which is differentiable 292 with respect to its inputs and does not require evolutionary information, could be used in conjunction with structure ${ }_{293}$ prediction tools to guide design of proteins with prescribed binding or non-binding properties (e.g. non-immunogenic 294 therapeutic proteins).

295 Finally, interpretable, end-to-end learning, combined with self-supervised learning techniques could pave the way 296 towards a complete dictionary of function-bearing structural motifs found in nature, deepening our understanding of ${ }_{297}$ the core principles underlying protein function.

\section{8 \\ ACKNOWLEDGEMENTS}

299 J.T. acknowledges financial support from the Edmond J. Safra Center for Bioinformatics at Tel Aviv University and 300 from the Human Frontier Science Program (cross-disciplinary postdoctoral fellowship LT001058/2019-C). D.S. was 301 supported by ISF 1466/18, Israel ministry of Science and Technology and HUJI-CIDR. This work was supported by ${ }_{302}$ Len Blavatnik and the Blavatnik Family Foundation. We are grateful to Sonia Lichtenzveig Sela and the CS system ${ }_{303}$ team for their technical support. We thank Raphaël Groscot for his help on pythreejs visualizations. We thank 304 Michael Nissan, Yoav Lotem, Mark Rozanov, Lirane Bitton, Matan Halfon and Shon Cohen for helpful discussions. AVAILABILITY

306 Data sets and source codes for training and evaluating ScanNet will be released upon publication. 


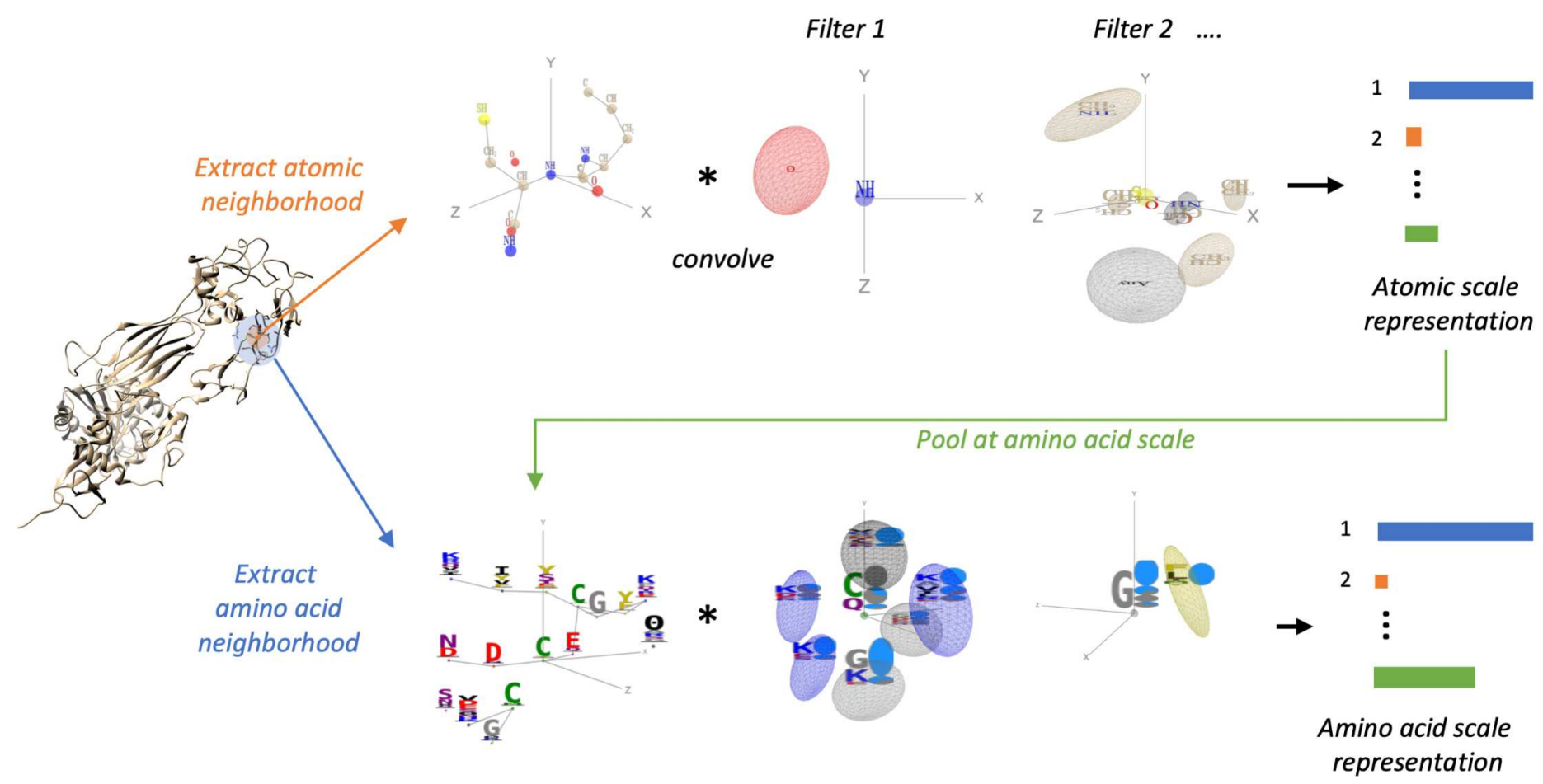

FIG. 1. Overview of the ScanNet architecture ScanNet inputs are the primary sequence, tertiary structure, and, optionally, position-weight matrix (PWM) computed from a multiple sequence alignment (MSA) of evolutionary-related proteins. Firstly, for each atom, neighboring atoms are extracted from the structure and positioned in a local coordinate frame (top left). The resulting point cloud is passed through a set of trainable, linear filters detecting specific spatio-chemical arrangements (top middle), yielding an atomic scale representation (top right). After aggregation of the atomic representation at amino acid (AA) level and concatenation with AA attributes, the process is reiterated with AA to obtain a representation of AA (bottom). The later is projected and locally averaged for residue-wise classification. 


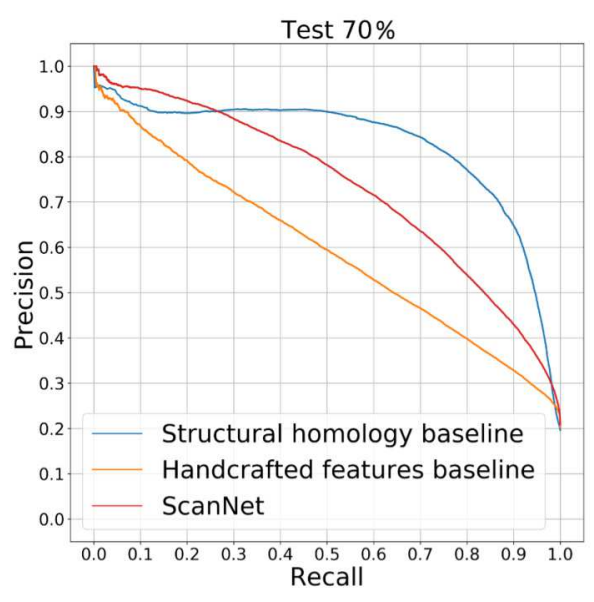

(a)

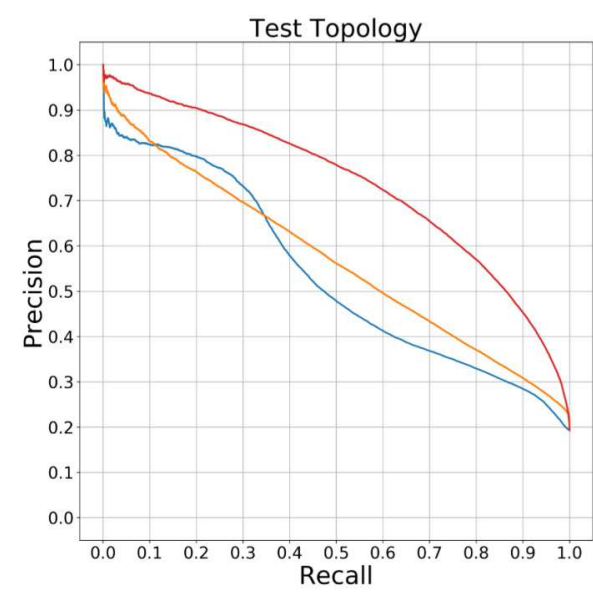

(c)

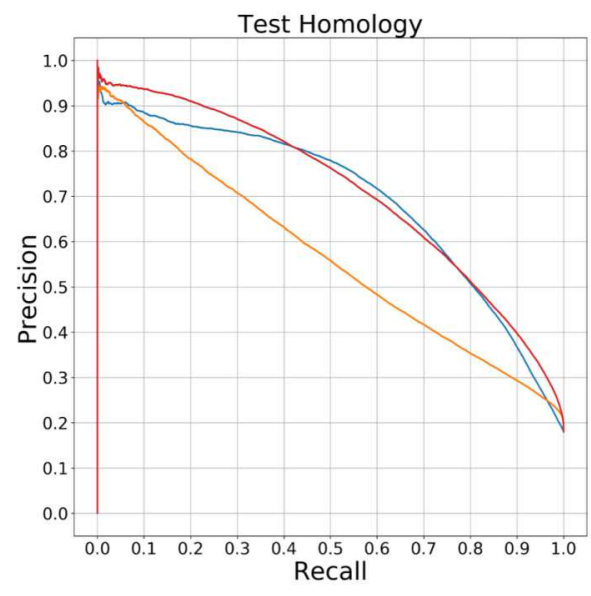

(b)
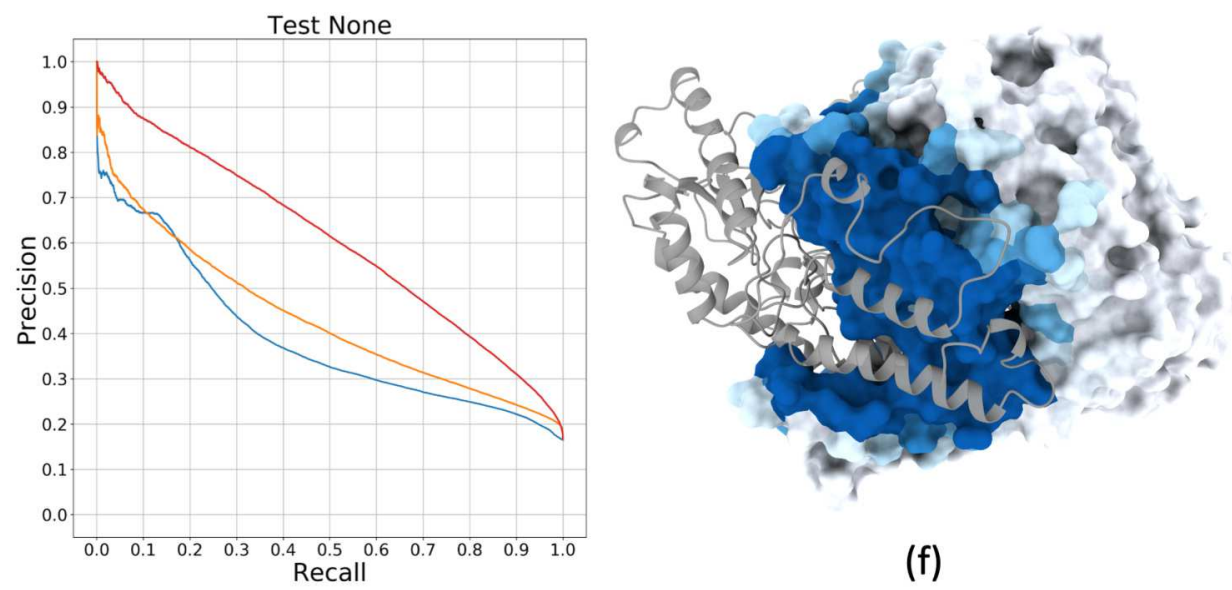

(f)

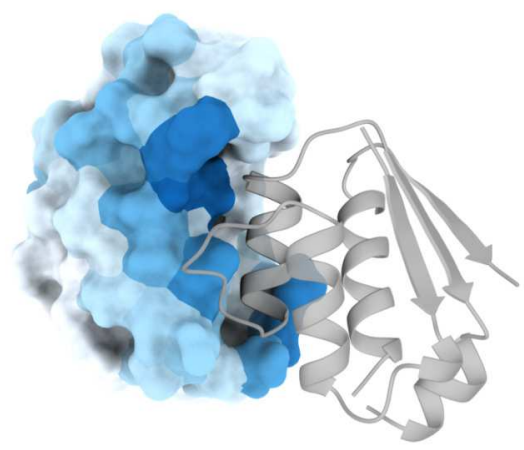

(e)

(d)

FIG. 2. Prediction of Protein-Protein Binding Sites (PPBS) with ScanNet (a)-(d) Precision-Recall curves of PPBS prediction for ScanNet, Structural homology, and Handcrafted features baseline methods (see main text). Train and test sets constructed from the redundant Dockground template database [45]. Proteins of the test set are subdivided into four nonoverlapping groups (a) Test 70\%: At least 70\% sequence identity with at least one train set example. (b) Test Homology: At most $70 \%$ sequence identity with any train set example, at least one train set example belonging to same protein superfamily (H level of CATH classification [48]). (c) Test Topology: At least one train set example with similar protein topology (T level of CATH classification [48]), none with similar protein superfamily. (d) Test None: None of the above. (e),(f) Illustration of predicted PPBS for (e) an enzyme (barnase, PDB ID: 1brs:A [56], Val. Homology dataset) with its inhibitor overlaid and (f) an homodimer (glutamic acid decarboxylase GAD67, PDB ID: 2okj:A [57], Test Topology dataset). The molecular surface of the query protein is shown with coloring based on predicted probability, ranging from low (white) to high (dark blue). The partner protein is shown in cartoon representation (gray transparent). Visualization software: ChimeraX [58] 
(a)

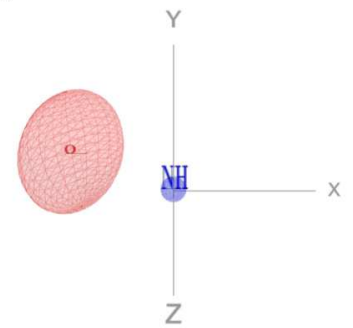

(b)

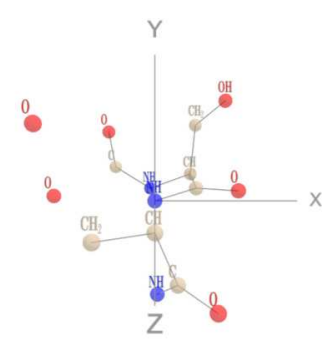

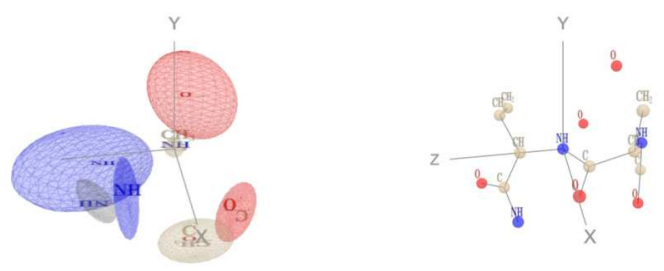

(c)

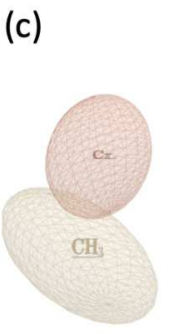

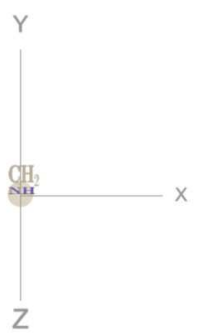

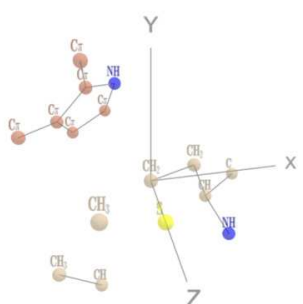

(d)

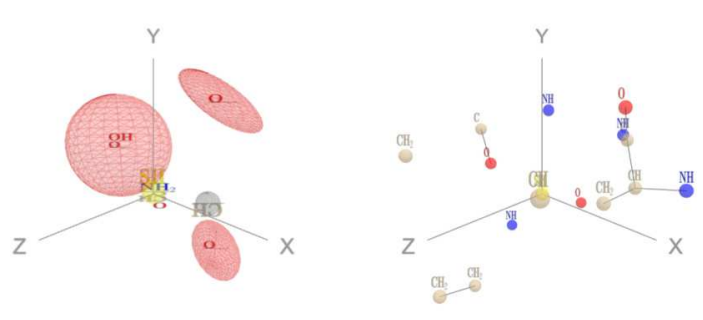

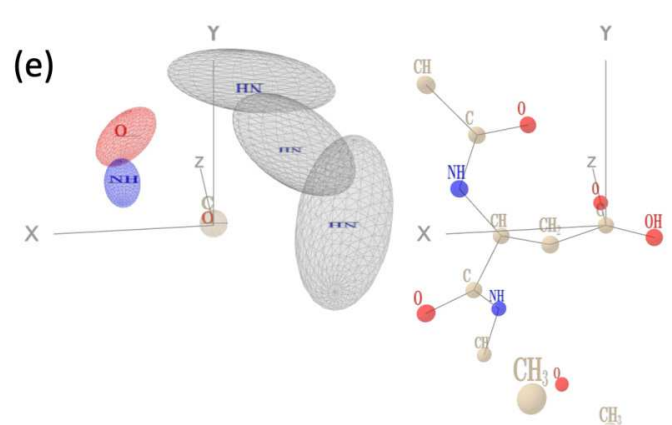

Coordination number

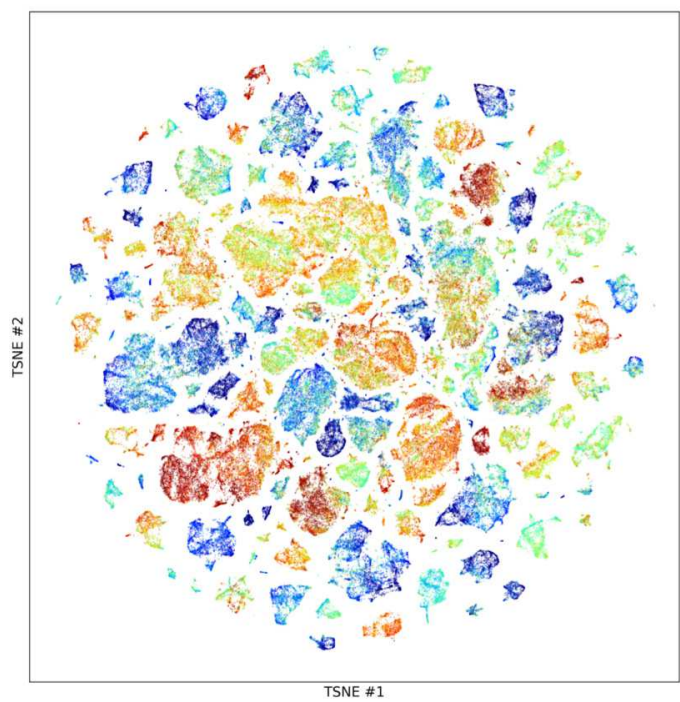

(g) (f)

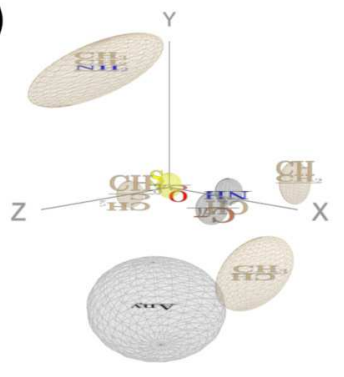

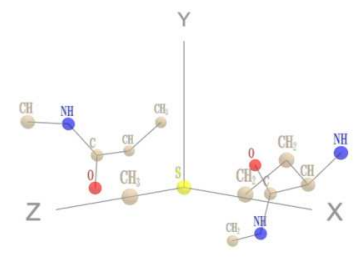

Electrostatic potential

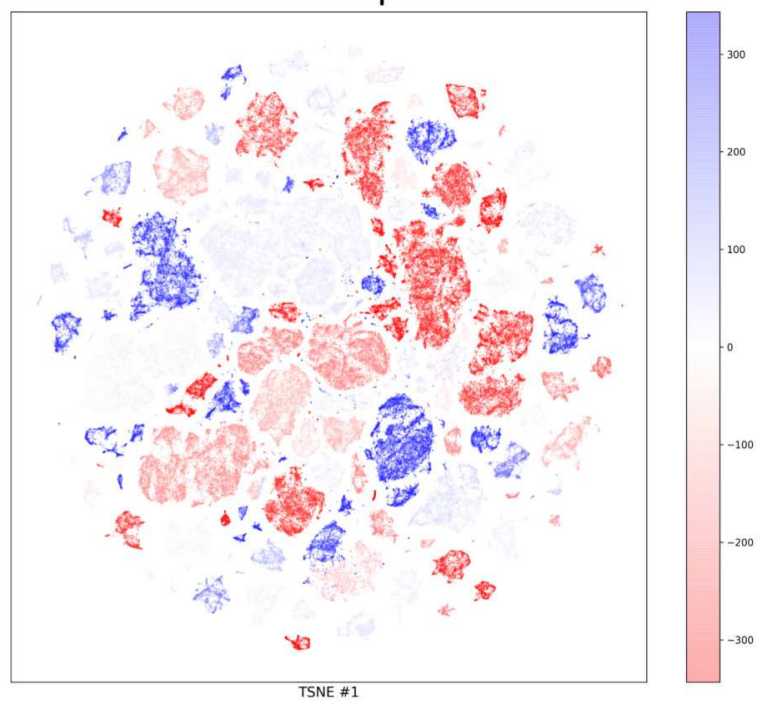

(h)

FIG. 3. Visualisation of the learnt atomic representation The panels (a)-(f) show each one spatio-chemical pattern on the left and one corresponding top-activating neighborhood on the right. Each pattern is depicted as follows: only the gaussian kernels relevant to the pattern are shown; they are represented by their unit ellipsoid. The corresponding locationwise attribute specificity is depicted as a weight logo inside the ellipsoid, similar to a position weight matrix: attributes with non-zero weights are stacked on top of one another with letter height proportional to their algebraic weight value, sorted from strongest positive (top) to strongest negative (bottom, reversed letters). The unit ellipsoid is colored based on the maximally activating attribute type if it is positive, or gray otherwise. Color code: carbon (beige), oxygen (red), nitrogen (blue), sulfur (yellow). The frame is overlaid in gray, with axes extending over $3.75 \AA$ A. Each filter/neighborhood pair is oriented independently for clarity. Visualizations created with pythreejs. (g), (h) Two-dimensional projection of the learnt atomic scale representation using T-SNE [59]. Each point corresponds to one atom of a representative set of proteins. Coloring based on atom coordination index (g) or electrostatic potential at the atom location, computed using APBS [49] (h). 
(a)

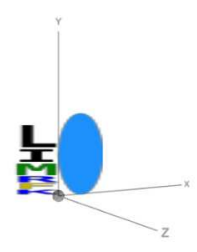

(c)

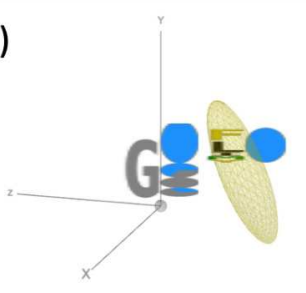

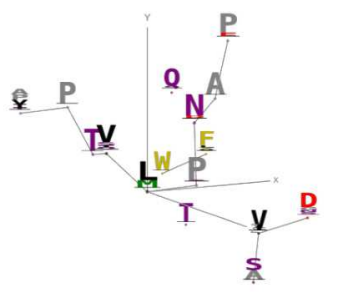

(b)

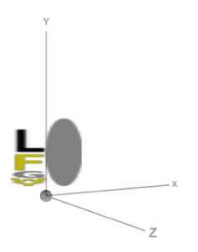

(d)
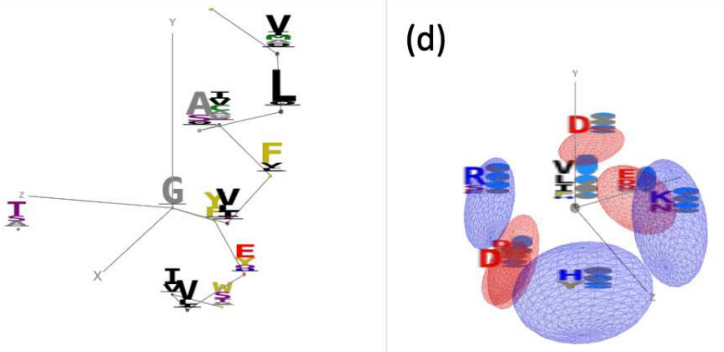
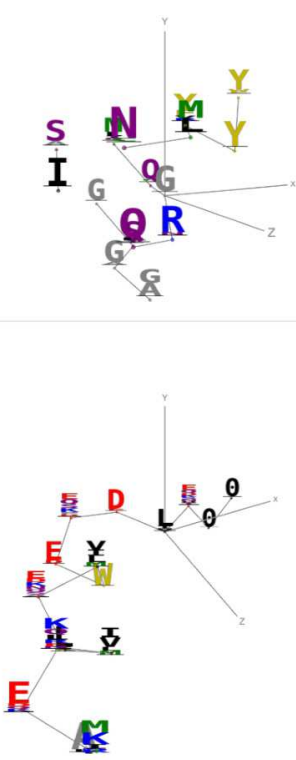

(e)
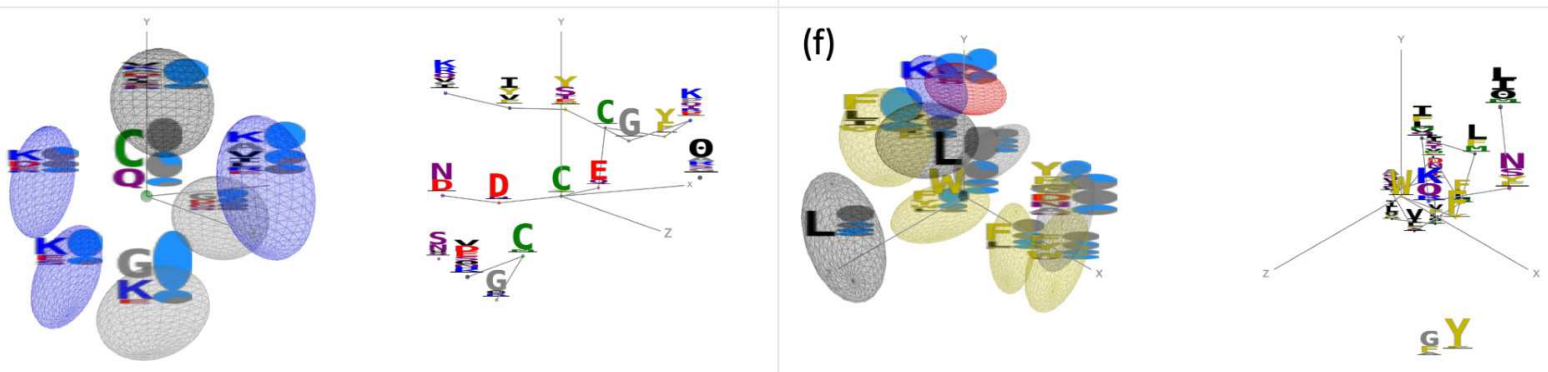

Secondary Structure

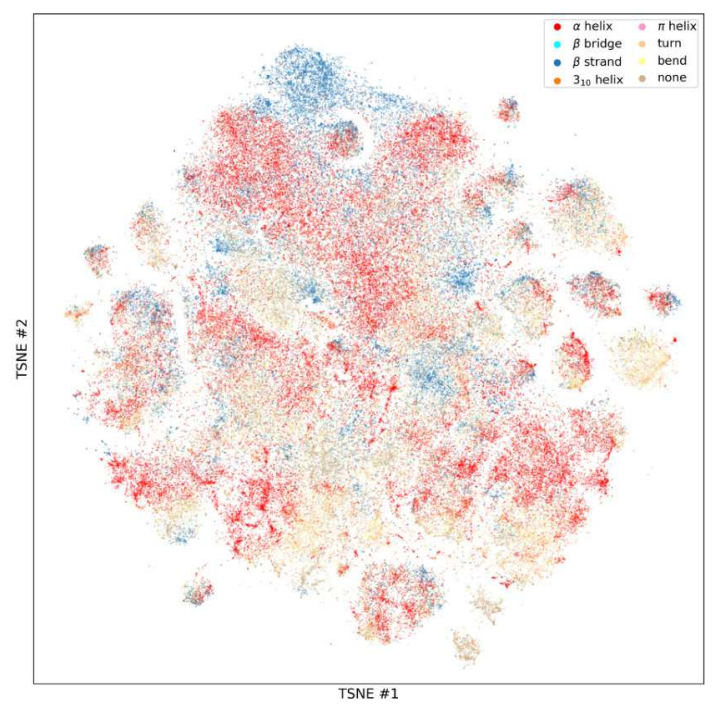

(g)

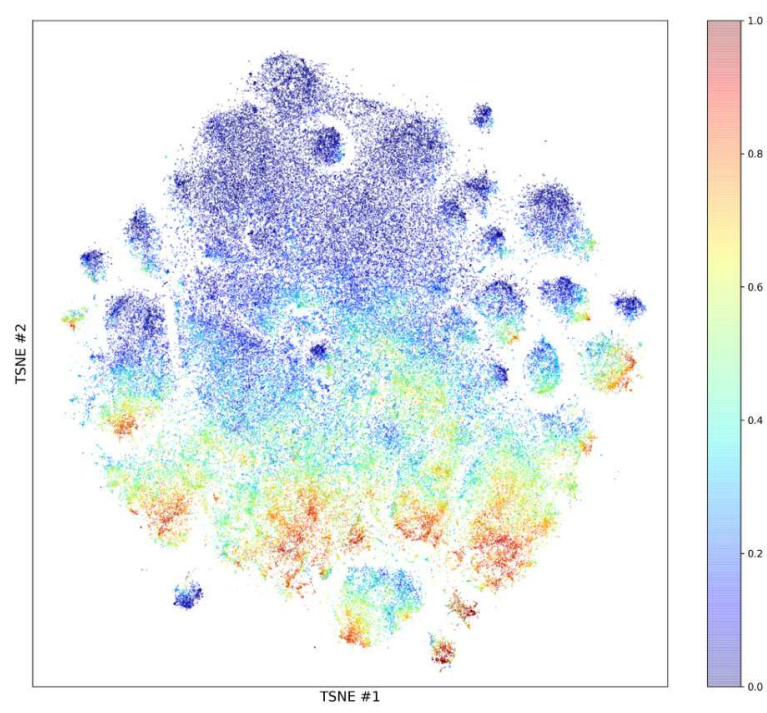

(h)

FIG. 4. Visualisation of the learnt amino acid representation The panels (a)-(f) show each one spatio-chemical pattern on the left and one corresponding top-activating neighborhood on the right. Gaussian kernels are depicted similarly as in Fig. 3. Since the input attributes are learnt, each component of a pattern is characterized by a complex specificity in attribute space. We represent it by the distributions of amino acid types and accessible surface areas of its top $1 \%$ maximally activating residues. The distributions are shown as a logo (each letter or symbol is proportional to the probability), with a total height proportional to the mean activation of the set. Accessible surface area values are discretized into four quartiles and represented as pie charts (from full gray $=$ buried to full blue = accessible). Amino acids are colored by chemical properties: negatively charged (red), positively charged (blue), polar (purple), hydrophobic (black), sulfur-containing (green), aromatic (gold), tiny/proline (gray). The frame is overlaid in gray, with axes extending over $9 \AA$. Each filter/neighborhood pair is oriented independently for clarity. Visualizations created with pythreejs. (g), (h) Two-dimensional projection of the learnt amino acid scale representation using T-SNE [59]. Each point corresponds to one amino acid of a representative set of proteins. Coloring based on secondary structure $(\mathrm{g})$ or accessible surface area $(\mathrm{h})$ calculated with DSSP [60]. Additional T-SNE plots available in Sup. Fig. S16. 


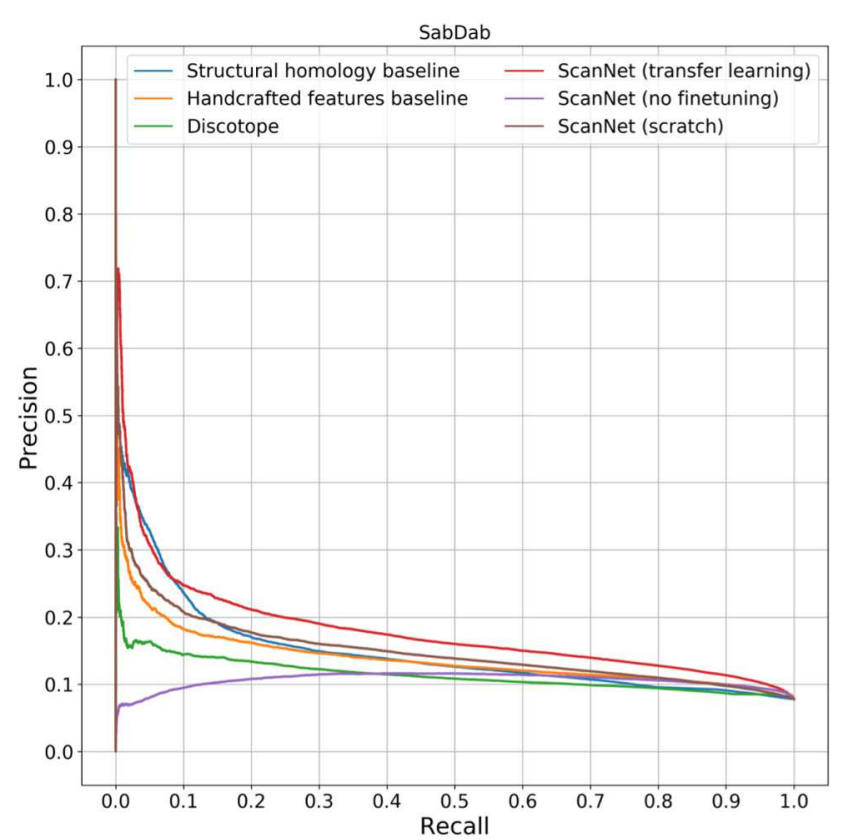

(a)

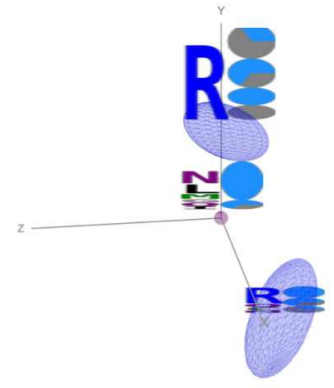

(b)

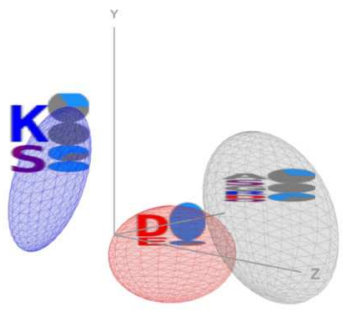

(d)

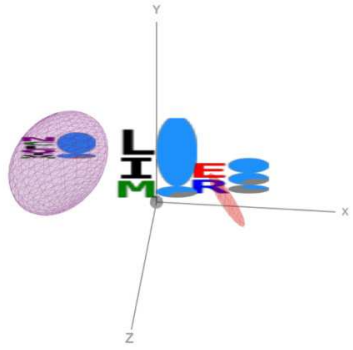

(c)

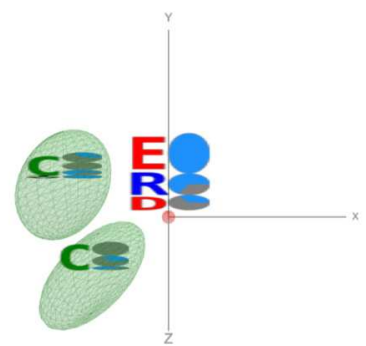

(e)

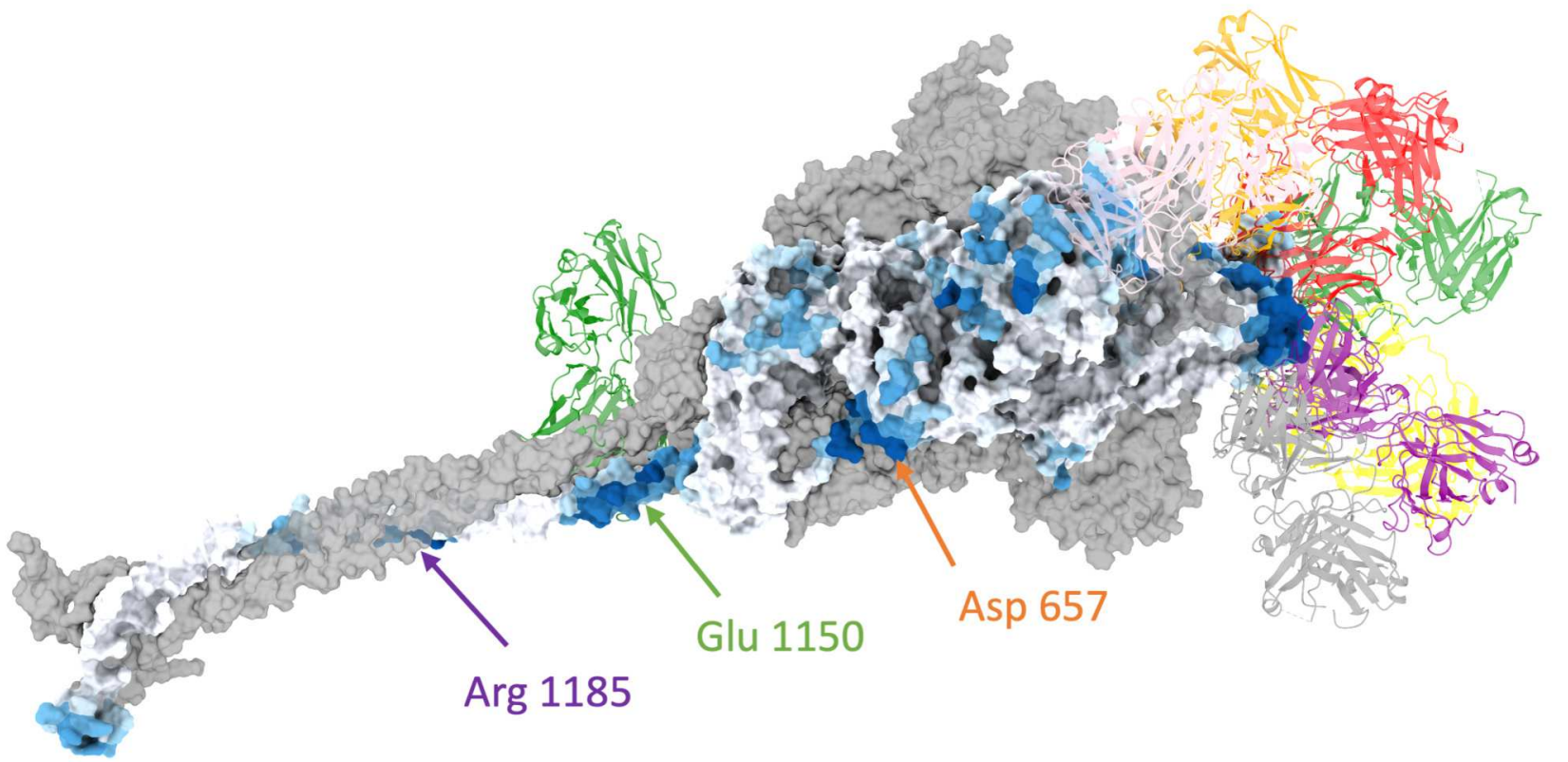

(f)

FIG. 5. Prediction of B-cell Epitopes with ScanNet (a) Precision-Recall curve of B-cell epitope prediction for baseline methods, Discotope [51] and ScanNet. Epitope database constructed from SabDab (timestamp: 04/19/2021) [50]; 5-fold crossvalidation performance is shown. (b)-(e) Selected learnt amino acid neighborhood filters whose activity is positively correlated with epitope probability. Same visualization as Fig. 4. (f) Application to Spike protein of SARS-CoV-2. Predictions performed on a MD snapshot of the spike trimer with one RBD open [61]. The monomer with open conformation is represented as molecular surface with color corresponding to BCE probability, from white (low) to dark blue (high). Representative antibodies binding the main epitopes are superimposed in color, cartoon representation, see full list in Sup. Table S5. 
308 The Materials and Methods is organized as follows. Section A) provides all mathematical and implementation details 309 for ScanNet. Section B) is dedicated to the baselines methods. Section C) covers data set construction, partition and 310 sample weights. In Section D), we evaluate the impact of induced fit on changes on ScanNet predictions. Section E) 311 contains additional results for the Protein-protein binding site and B-cell epitope prediction tasks.

314 PDB parsing PDB files are parsed using Biopython [62]. We gather, for each chain, the amino acid sequence 315 and the point cloud of heavy atoms, formally a list of triplets $\left\{\left(\operatorname{coordinates}_{l}, \operatorname{residue~}_{l}\right.\right.$, atom id $\left.\left._{l}\right) l \in\left[\left[1, N_{\text {atoms }}\right]\right]\right\}$, 316 e.g. ([10.1,101.3,-12.6], 97, CA). Only atoms belonging to classical residues are considered; exotic residues, additional 317 molecules bound to the chain (e.g. heme, ATP, glycosyl groups, ions...) are excluded.

${ }_{318}$ Towards definition of a local reference frame for each atom, we reconstruct the molecular graph (i.e. atom as nodes 319 and covalent bonds as edges) using the residue and atom ids. Each heavy atom has one, two or three neighbors on 320 the molecular graph; if it has only one (e.g. for methyl group $\mathrm{CH}_{3}$ ), a virtual hydrogen atom is appended to the 321 graph. Two neighbors are selected to define a triplet of points $\left(l, i_{\mathcal{N}_{1}(l)}, i_{\mathcal{N}_{2}(l)}\right)$ from which a frame can be derived. ${ }_{322}$ The coordinates of atoms $l, i_{\mathcal{N}_{1}(l)}$ and $i_{\mathcal{N}_{2}(l)}$ respectively define the center, $x z$ plane and $z$ direction, see paragraph ${ }_{323}$ on Frame Computation Module and Equation 3. The first ("previous") neighbor is chosen as the closest from the ${ }_{324}$ N-terminal nitrogen. For the second ("next") neighbor, if the atom has three neighbors, the furthest from the C325 terminal carbon among the remaining two is used [63]. For instance, the two neighbors of the $C_{\alpha}$ atom of residue $l$ ${ }_{326}$ are the $N$ of the residue $l-1$ and the $C$ of the residue $l$. The two neighbors of the $C_{\beta}$ atom are the $C_{\alpha}$ atom and the ${ }_{327} C_{\gamma}$ atom of the side chain.

${ }_{328}$ Also based on the molecular graph, an attribute is assigned to each heavy atom based on its type and the number of 329 bound hydrogens. Twelve categories are defined: $\mathrm{C}, \mathrm{CH}, \mathrm{CH}_{2}, \mathrm{CH}_{3}, \mathrm{C}_{\pi}$ (aromatic carbon), $\mathrm{O}, \mathrm{OH}, \mathrm{N}, \mathrm{NH}, \mathrm{NH} \mathrm{H}_{2}, \mathrm{~S}, \mathrm{SH}$. ${ }_{330}$ Overall, four atomic arrays are constructed:

331 - The point cloud of atoms and virtual atoms (float, size $\left[N_{\text {atoms }}+N_{\text {virtualatoms }}, 3\right]$ ).

332

- The triplets of indices for constructing atomic local frames (integer, size $\left[N_{\text {atoms }}, 3\right]$ ).

- The atom groups (integer, size $\left.\left[N_{\text {atoms }},\right]\right)$.

- The residue index of each atom (integer, size $\left.\left[N_{\text {atoms }},\right]\right)$.

\section{5}

For the amino acid level, four similar arrays are constructed. The point cloud consists of the $C_{\alpha}$ and the side 336 chain centers of mass (SCoM) of each amino acid. For Glycines - which do not have a sidechain - a virtual SCoM 337 is defined as $\mathbf{x}_{\mathbf{S C o M}}=3 \mathbf{x}_{C_{\alpha}}-\mathbf{x}_{C}-\mathbf{x}_{N}$, where $\mathbf{x}_{C_{\alpha}}, \mathbf{x}_{C}, \mathbf{x}_{N}$ denote the coordinate of respectively the $C_{\alpha}$ atom of 338 the residue, the $N$ atom of the previous residue and the $C$ atom of the residue. The reference frame of each amino 339 acid is defined by the $C_{\alpha}$ (center), previous $C_{\alpha}$ along the backbone (xz-plane) and SCoM (z-axis). Previous works ${ }_{340}[24,30]$ considered other amino acid frames constructed from the backbone atoms only. Here, our rationale was that 341 neighboring amino acids located in the opposite direction from the side chain (i.e. the interior of the protein) should 342 not matter for functionality. It also facilitates filter interpretation, as for exposed residues the side-chain points ${ }_{343}$ towards the exterior of the protein. We also experimented frames constructed from consecutive $C_{\alpha}$ and found no 344 difference performance-wise, but have not visualized the corresponding filters.

345 The per-residue attribute is given by the position-weight matrix (21-dimensional probability distribution, see below) 346 or the one-hot-encoded sequence for the models without evolutionary information.

347 Derivation of the Position Weight Matrix Given the sequence, we first construct a Multiple Sequence Align348 ment (MSA) by homology search using HHblits 2 (4 iterations, default values of other parameters) [64] on the Uni${ }_{349}$ Clust30_2018_06 database [65] (except for the SARS-Cov-2 Spike Protein for which we used the UniRef30_2020_06). 350 Next, a sequence dependent weight $w(S)$ was computed so as to i) address sampling redundancy [66] and ii) focus the 351 alignment around the wild type [67]:

352

$$
w(S)=\frac{1}{\text { Number of 90\% sequence identity homologs }} \times \exp \left(-\frac{D_{\text {Hamming }}(S, W T)}{d_{0}}\right),
$$


353 where $d_{0}$ is adjusted such that the effective number of samples is $B_{\text {eff }} \equiv \sum_{S} w(S)=500$. If the alignment is 354 initially too small, $d_{0}=\infty$ is used. Focusing the alignments allows to detect local evolutionary conservation patterns 355 as opposed to family-level conservation patterns; this is relevant as protein-protein interfaces are not always conserved 356 at the superfamily level.

358 Notations The following notations are used throughout presentation of the modules; $x$ : Global coordinates; $f$ : 359 Frames; $x^{\ell}$ : Local coordinates; $a$ : Attributes; $a^{\ell}$ : Local attributes; $L$ : Size of point set; $K$ : Number of points in a 360 neighborhood; $D$ : Dimension of coordinates; $N / M$ : Dimension of attributes. G: Number of Gaussian kernels. All 361 upper case letters are integer dimension numbers. The corresponding lower case letter denote running indices e.g. ${ }_{362} a_{l n}$ denotes the $n^{\prime}$ th $(n \in \llbracket 1, N \rrbracket)$ attribute of the $l^{\prime}$ th $(l \in \llbracket 1, L \rrbracket)$ point of the point cloud and $x_{l k d}^{\ell}$ is the $d^{\prime}$ th local 363 coordinate of the $k$ 'th neigbhor of point $i$. Bold letters denote vectors or matrices.

${ }_{364}$ Attribute Embedding Module (AEM) applies an element-wise non-linear transformation to the attributes $a_{l n}$ 365 of each point. Here, we used a element-wise dense layer, i.e. a matrix product followed by ReLU non-linearity for all 366 AEM except for the initial atomic attribute embedding module - for which the input is a categorical variable and a 367 one-hot encoding layer is applied. The equation for the AEM writes:

$$
a_{l m}^{\prime}=\operatorname{ReLU}\left[\sum_{n} a_{l n} w_{n m}+\theta_{m}\right]
$$

${ }_{369}$ Frame Computation Module (FCM) takes as input a point cloud $x_{l d}$ and a set of triplets of indices $\left(i_{l 1}, i_{l 2}, i_{l 3}\right)$ 370 and calculates, for every triplet, a frame $f_{l d d^{\prime}}$ of size $\left.[L, 4,3]\right)$, constituted by the center and the three unit vectors. 371 The equation writes:

$$
\begin{aligned}
\mathbf{f}_{11}(\text { center }) & =\mathbf{x}_{\mathbf{i}_{11}} \\
\mathbf{f}_{14}(\mathrm{z}-\text { axis }) & =\frac{\mathbf{x}_{\mathbf{i}_{13}}-\mathbf{x}_{\mathbf{i}_{11}}}{\left\|\mathbf{x}_{\mathbf{i}_{13}}-\mathbf{x}_{\mathbf{i}_{11}}\right\|} \\
\mathbf{f}_{13}(\mathrm{y}-\text { axis }) & =\frac{\mathbf{f}_{14} \times\left(\mathbf{x}_{\mathbf{i}_{12}}-\mathbf{x}_{\mathbf{i}_{11}}\right)}{\left\|\mathbf{f}_{14} \times\left(\mathbf{x}_{\mathbf{i}_{12}}-\mathbf{x}_{\mathbf{i}_{11}}\right)\right\|} \\
\mathbf{f}_{12}(\mathrm{x}-\text { axis }) & =\frac{\mathbf{f}_{13} \times \mathbf{f}_{14}}{\left\|\mathbf{f}_{13} \times \mathbf{f}_{\mathbf{l}}\right\|}
\end{aligned}
$$

373 Where $\times$ denotes the cross-product. Examples of frames overlaid on a protein structure are shown in Sup. Fig. S1 374 (a,b). The FCM has no trainable parameters.

375 Neighborhood Computation Module (NCM) determines, for each point, its $K$ closest neighbors in space 376 (including itself), computes their local coordinates and duplicates their attributes. Its inputs are a set of frames $f_{\text {lid }}$ 377 and attributes $a_{l n}$, and outputs are the neighborhoods $x_{l k d}^{\ell}, a_{l k n}^{\ell}$. The nearest neighbor search is implemented naively 378 by computing distances between all pairs of frame centers. For the atomic and amino acid neighborhoods, we use 379 as local coordinates the three euclidean coordinates of the second frame center in the first frame and take $K=16$. 380 For the neighborhood attention module, we take $K=32$ and use five coordinates: the distance between both frames 381 centers $\left\|\mathbf{f}_{\mathbf{l 1}}-\mathbf{f}_{\mathbf{1 1}}^{\prime}\right\|$, the dot product between the side-chain directions $\mathbf{f}_{\mathbf{1 4}} \cdot \mathbf{f}_{\mathbf{1 4}}^{\prime}$, the dot product between the side-chain 382 directions and the center to center vectors $\mathbf{f}_{\mathbf{1 4}} \cdot \frac{\mathbf{f}_{11}^{\prime}-\mathbf{f}_{11}}{\left\|\mathbf{f}_{11}^{\prime}-\mathbf{f}_{\mathbf{1 1}}\right\|}$ (and symmetric) and the distance between amino acids along 383 the sequence (clipped at $d_{\max }=8$ ). They are shown respectively as $d, \omega, \theta, \theta^{\prime}, d_{\text {sequence }}$ in Sup. Fig. S1 (c); The NCM 384 has no trainable parameters.

385 Neighborhood Embedding Module (NEM) is the core module of ScanNet. NEM convolves each neighborhood 386 with a set of trainable spatio-chemical filters, akin to convolutional filters in image CNNs (Fig. 1). Its inputs are a 387 set of $K$ points with local coordinates $x_{k d}^{\ell}$ and attributes $a_{k n}^{\ell}$, where $k \in[1, K], d \in[1, D], n \in[1, N]$ respectively 388 denote neighbor, coordinate and attribute indices. NEM outputs a set of $M$ filter activities $y_{m}$. It is parameterized звя using $G=32$ gaussian kernels (as in [68]) and a bilinear product as follows:

$$
y_{m}=\operatorname{ReLU}\left[\sum_{k, g, n} W_{m g n}^{s c} \mathcal{G}\left(\mu_{\mathbf{g}}, \boldsymbol{\Sigma}_{\mathbf{g}}, \mathbf{x}_{\mathbf{k}}\right) a_{k n}+\sum_{k, g} W_{m g}^{s} \mathcal{G}\left(\mu_{\mathbf{g}}, \boldsymbol{\Sigma}_{\mathbf{g}}, \mathbf{x}_{\mathbf{k}}\right)+W_{m}^{b}\right]
$$


391 Where $\mathcal{G}(\mu, \boldsymbol{\Sigma}, \mathbf{x})=\exp \left[-\frac{1}{2}(\mathbf{x}-\mu)^{T} \boldsymbol{\Sigma}^{-1}(\mathbf{x}-\mu)\right]$ is a Gaussian kernel of center $\mu$ and (full) covariance matrix $\Sigma$, 392 and $\mathbf{W}^{\mathbf{s c}}, \mathbf{W}^{\mathbf{s}}, \mathbf{W}^{\mathbf{b}}$ are trainable tensors of sizes $[M, G, N],[M, G],[M$,$] . See a graphical sketch in Sup. Fig. S1 (d).$ ${ }_{393}$ The gaussian kernels are trainable and shared between all filters of a given layer, see implementation in Sup. Fig. S1 394 (e).

395 The above parameterization offers several advantages over other choices such as Multilayer perceptrons [25, 69, 70] 396 or spherical harmonics [35, 71]. First, it is straightforward to interpret: a filter $m$ with large entries of the tensor ${ }_{397} \mathbf{W}^{\text {sc }}$ for some $g, n$ is positively activated by points having attribute $n$ and located near the center of the Gaussian $g$. 398 Similarly, the matrix $W^{s}$ encodes attribute-independent spatial sensitivity and $W^{b}$ is a bias vector. Second, localized 399 filters, i.e. filters detecting only one or few combinations of point/attributes can be obtained by simply enforcing ${ }_{400}$ sparsity of the weights $W^{s c}$ and $W^{s}$ via a regularization penalty. Third, the filters are guaranteed to have an almost 401 compact support, as the gaussian functions decay rapidly as $\|x\| \rightarrow \infty)$. This ensures that the diameter of the 402 neighborhood is effectively capped irrespective of the local point density - in particular for unpacked or disordered ${ }_{403}$ regions. Last but not least, the gaussian kernels can be initialized using unsupervised learning, thereby improving ${ }_{404}$ performance and limiting run-to-run performance variance (initialization protocol detailed below).

405 For the sparsity regularization, we use the following combination of cost function and norm constraint:

406

$$
\begin{aligned}
& \mathcal{R}_{1}^{2}\left(\mathbf{W}^{\mathbf{s c}}\right)=\frac{\lambda_{1}^{2}}{2 G N} \sum_{m}\left(\sum_{g n}\left|W_{m g n}^{s c}\right|\right)^{2} \\
& \mathcal{R}_{1}^{2}\left(\mathbf{W}^{\mathbf{s}}\right)=\frac{\lambda_{1}^{2}}{2 G} \sum_{m}\left(\sum_{g}\left|W_{m g}^{s}\right|\right)^{2} \\
& \sqrt{\sum_{g n}\left(W_{m g n}^{s c}\right)^{2}}=\sqrt{\frac{G}{K}}, \forall m
\end{aligned}
$$

${ }_{407}$ The so-called $L_{1}^{2}$ regularization (as previously described in [72]) is a variant of the $L_{1}$ regularization $\left(\mathcal{R}_{1}\left(\mathbf{W}^{\mathbf{1}}\right)=\right.$ $\left.{ }_{408} \sum_{m g n}\left|W_{m g n}^{1}\right|\right)$ that promotes homogeneity of the filter sparsity values. This can be seen from the expression of the 409 gradients, which write:

410

$$
\frac{\partial \mathcal{R}_{1}^{2}}{\partial W_{m g n}^{s c}}=\left(\frac{\lambda_{1}^{2}}{G N} \sum_{g n}\left|W_{m g n}^{s c}\right|\right) \operatorname{sign}\left(W_{m g n}^{s c}\right)
$$

$$
\frac{\partial \mathcal{R}_{1}}{\partial W_{m g n}^{s c}}=\quad \lambda_{1} \quad \operatorname{sign}\left(W_{m g n}^{s c}\right)
$$

${ }_{411}$ The $L_{1}^{2}$ regularization is effectively a $L_{1}$ regularization with a filter-dependent regularization strength: filters that ${ }_{412}$ are sparse (resp. not sparse) have a small (resp. large) $L_{1}$ norm, hence a small (resp. large) effective $L_{1}$ regularization ${ }_{413}$ strength; which in turn further relaxes or tightens the sparsity constraint. The $L_{2}$ filter norm constraint is necessary ${ }_{414}$ to ensure a well-defined optimization problem because of the downstream batch norm layers. Indeed, the operation ${ }_{415} W_{m g n}^{1} \rightarrow \rho_{m} W_{m g n}^{1}$ leaves the final output invariant, as it is exactly compensated by the covariation of the slope of 416 the subsequent batch norm layer through $\alpha_{m} \rightarrow \frac{\alpha_{m}}{\rho_{m}}$ (using notations from [73]). Therefore, without constraint the ${ }_{417}$ optimum would be the asymptote $W_{m g n}^{1} \rightarrow 0, \alpha_{m} \rightarrow \infty$ with $W_{m g n}^{1} \times \alpha_{m}=W_{m g n}^{1 \star}$, the optimum weight value without 418 any regularization. The norm value is chosen such that the filter output $y_{m}$ (Eqn. 4 ) has roughly variance 1 when the 419 attributes have variance 1.

${ }_{420}$ To determine the value of the regularization penalty $\lambda_{1}^{2}$, we searched for a satisfying compromise between inter${ }_{421}$ pretability (localized filters) and classification performance. We first determined the order of magnitude of $\lambda_{1}^{2}$ as 422 follows: assuming filters weights $W^{1}$ with sparse entries (a fraction $p$ of non-zero weight, with typical weight value ${ }_{423} W$ ), the $L_{2}$ norm writes $\|W\|_{2}=\sqrt{p G N} W \equiv \sqrt{G / K}$, i.e. $W \sim \frac{1}{\sqrt{K N p}}$ and $\mathcal{R}_{1}^{2} \sim \frac{\lambda p G M}{2 K}$. Further assuming that the ${ }_{424}$ regularization penalties and cross-entropy variations (about $10^{-2}$ per site in our experiments) should approximately 425 balance each other, and with $G / K=2, M=128$ for both atomic and amino acid filters, we find that $\lambda \sim 10^{-2} / p M$. ${ }_{426}$ With a target $p \sim 10^{-2}$, we conclude that $\lambda_{1}^{2} \sim 10^{-2}$. After experimentation, we chose $\lambda_{1}^{2}=2.10^{-3}$ for both atomic ${ }_{427}$ and amino acid filters, as this value yielded the most satisfactory filter visualizations and prediction performances.

${ }_{428}$ Atomic to Amino Acid Pooling Towards calculation of residue-wise outputs, the learnt atomic scale represen429 tation must be aggregated at the amino acid scale. We recall that the constituting atoms of an amino acid may play 430 different functional roles, hence symmetric pooling operations may not be sufficiently expressive. ScanNet instead ${ }_{431}$ employs a trainable multi-headed attention pooling. It writes: 
${ }_{433}$ Where $\mathbf{P}, \mathbf{A}$ are trainable projection and attention weighting) matrices. Eqn. 7 generalizes the average pooling ${ }_{434}\left(A_{m, .}=0\right)$ and maximum pooling $\left(A_{m, .}=\alpha P_{m}\right.$. with large $\left.\alpha\right)$ operations. A sparsity regularization is also employed ${ }_{435}$ for both $\mathbf{P}, \mathbf{A}$ to simplify correspondence between atomic and amino acid filters.

${ }_{436}$ Neighborhood Attention Module (NAM) computes spatially coherent, residue-wise output probabilities from ${ }_{437}$ amino acid frames and spatio-chemical filter activities. The computation is done in four stages, see Sup. Fig. S2. First, 438 local amino acid scale neighborhoods of size $K=32$ are constructed, with graph-type local coordinates: distances, 439 angles and sequence distances (see Sup. Fig.S1(c)). Second, the five-dimensional edges are projected element-wise 440 into a single algebraic value using trainable Gaussian kernels followed by a dense layer with linear activation function. ${ }_{441}$ No bias is used for the dense layer, such that the edge value decays to zero as the distance increases. Third, the ${ }_{442}$ filter activities are projected to scalar values and locally averaged using attention-based weights. Our expression of 443 the weighting coefficients slightly differs from the graph attention network formulation of [41] as follows: each node 444 is characterized by a trainable output feature (unnormalized binding site probability), self-attention ("passenger" ${ }_{445}$ residues should have weak self-attention), cross-attention (hotspots should have strong cross-attention) and contrast ${ }_{446}$ coefficients (residues can follow either the majority or the hotspot residue). The weights may also take negative values ${ }_{447}$ depending on the edge values. Finally, a logistic function is applied to obtain normalized probabilities.

449 A diagram showing the architecture of the network is drawn in Sup. Fig. S3, and a table listing each module with 450 its input(s) and output(s) sizes and comments is provided as Supplementary Data. In total, the network contains ${ }_{451} 475 \mathrm{~K}$ parameters, of which about $200 \mathrm{~K}$ are non-zero.

453 Initialization For the neighborhood embedding modules, the gaussian kernels were initialized by unsupervised 454 learning; using a subset of the training set, we computed atomic and amino acid neighborhoods, and fitted the spatial 455 point density using a Gaussian Mixture Model (as implemented in Scikit-learn [74], best of 10 runs with Kmeans++ 456 initialization, full covariance matrix and $10^{-1}$ covariance matrix regularization). For the trainable graph edges of the ${ }_{457}$ Neighborhood Attention module computed from distances and angles, we initialized them as a least square parametric ${ }_{458}$ fit of the label autocorrelation function (normalized):

459

$$
A(\text { distance }, \text { angles }, \ldots)=\frac{\left(\mathbb{E}\left[Y_{i} Y_{j} \mid d_{i j}=\text { distance }, \ldots\right]-\mathbb{E}\left[Y_{i}\right]^{2}\right)}{\mathbb{E}\left[Y_{i}\right]-\mathbb{E}\left[Y_{i}\right]^{2}}
$$

All remaining weights are initialized using symmetric random distributions, see details in supporting table.

${ }_{461}$ Padding and protein serialization trick In our implementation, ScanNet takes as input an entire protein ${ }_{462}$ and computes neighborhoods on-the-fly, akin to a fully convolutional segmentation network [75]. Training on GPUs ${ }_{463}$ requires fixed size inputs but the lengths of proteins varied by almost two orders of magnitude in our dataset (see Sup. ${ }_{464}$ Fig. S4 (e) ). To avoid truncating large proteins or wasting most of the computational power, we used the following ${ }_{465}$ protein serialization trick. We choose a relatively large maximal protein length $\left(L_{\max }=1024,2120\right.$ for the PPBS 466 and $\mathrm{BCE}$ datasets), concatenate several proteins into a single example and translate each protein far away from the ${ }_{467}$ others, such that no two proteins overlap in space. Since ScanNet exploits only local neighborhoods, the predictions ${ }_{468}$ for each protein are fully independent from one another. Before training or prediction, we group proteins in a greedy 469 fashion that minimizes the unused placeholders. Proteins are first sorted by length and the largest ones are first 470 picked; then, we pick among the remaining proteins the largest that fits into the placeholder (if any), concatenate 471 it, and continue until the placeholder is full. For the PPBS dataset, we found that about $96 \%$ of the amino acids 472 placeholders were used, as opposed to less than $25 \%$ with naive padding. This results in a speed-up of about 4 -fold. ${ }_{473}$ Finally, we used masking layers across the network to prevent backpropagating errors for the remaining placeholders 474 that do not contain any residue. 
${ }_{475}$ Optimization The network is trained by minimizing the binary cross-entropy loss function by backpropagation 476 using the ADAM optimizer [76]. We set the maximum number of epochs to 100, the batch size to 1, the learning rate 477 to $10^{-3}\left(10^{-4}\right.$ for the transfer learning) and perform learning rate annealing and early stopping based on the validation 478 cross-entropy; the optimal model was usually reached before 10 epochs. We used batch normalization layers before 479 each ReLU non-linearity throughout the network to avoid vanishing gradients. Finally, regarding sample weighting, a ${ }_{480}$ complication of the protein serialization trick is that residues of a single example may have different sample weight as ${ }_{481}$ they come from different proteins. To account for this, we formally replaced the binary cross-entropy loss function and ${ }_{482}$ logistic non-linearity with a categorical cross-entropy and softmax function with two output classes; training labels ${ }_{483}$ are multiplied by their weight so as to replicate the weighted loss function.

${ }_{484}$ Software and runtime The network was implemented using Tensorflow v1.14.0 [77] and Keras v2.2.5 [78]. Training ${ }_{485}$ was completed in about one to two hours using a single Nvidia V100 GPU. The inference time is dominated by the ${ }_{486}$ construction of the MSA and the calculation of the Position Weight Matrix - it is of the order of one to few minutes ${ }_{487}$ depending on sequence length and MSA depth.

\section{2}

493 $511(5,10,20)$, minimum child weight $(5,10,50,100), \gamma(0.01,0.1,1.0,5),. \eta(0.5,1.0)$.

${ }_{513}$ Several approaches leveraging sequence and structure homology were previously developed [5-11], but were not ${ }_{514}$ readily available for large scale benchmarking, which prompted us to develop an in-house structural homology baseline 515 method. It features three key components:

- Amino acid type (one-hot encoded, 20 dim.).

- Secondary structure type (one-hot encoded, 8 dim.); computed with DSSP [60].

- Relative Accessible Surface Area (1 dim.); computed with DSSP [60].

- Coordination Number (1 dim.), defined as the number of $C_{\alpha}$ atoms in a ball of radius 13 center around the $C_{\alpha}$ atom of the amino acid.

- Half Sphere Exposure Index [79] (1 dim.), defined as follows: let $N_{1}$ be the coordination number, and $N_{2}$ the number of $C_{\alpha}$ atoms in the intersection of a ball of radius 13 center and above the plane defined by the $C_{\alpha}-C_{\beta}$ vector. The half sphere exposure index is $\frac{2 N_{2}-N_{1}}{N 1} \in[-1,1]$.

- Backbone and Sidechain Depth [80] (2 dim.). The molecular surface was computed using MSMS (probe radius $1.5 \AA$ ) [81], and the distance to the surface was computed and averaged for all backbone (resp. sidechain) atoms.

- Surface convexity index (3 dim.) [82]. For each atom, we construct a ball of radius 5/8/11 Åcentered on it, and compute the $f$ fraction of its volume located on the inside of molecular surface; the index is given by $2 f-1 \in[-1,-1]$. The surface convexity index is averaged at amino acid level.

- Position Weight Matrix (PWM, 21 dim.)

- Conservation score $C=\log 21+\sum_{a} \log P W M(a)$ (1 dim.).

In total, 58 features are used. For classification, we used the xgboost algorithm (boosted trees) [46]. The classifier was trained by cross-entropy minimization, using the same training and validation sets. We used 100 boosting rounds (with early stopping on validation loss), and the following four parameters were determined by grid search: tree depth

2. Structural homology baseline

1. A non-redundant database of template protein chains with known binding sites. We used here as template the training set of ScanNet for a fair comparison. The template database was further clustered at the $90 \%$ (resp. 95\%) sequence identity for the PPBS and BCE datasets, for speed gain purposes and to simplify alignment weighting, see below.

\section{B. Baseline Methods}

\section{Handcrafted features baseline}

For the handcrafted features baseline, we computed for each amino acid geometric, chemical and evolutionary features as described in recent works on prediction of protein-protein / protein-antibody binding sites [12-18]. The following features were computed: 

535 alignment matrix:

s. that $P_{l}=P_{0}$ if no alignment is found for a given residue. The log-weight function $W$ is parameterized by 541 a two-layer perceptron with 20 hidden nodes and hyperbolic tangent activation function and was trained by cross${ }_{542}$ entropy minimization on a subset of the validation set; after training, we found that $\mathcal{W}$ is a increasing function of 543 both alignment coverage and sequence identity, in agreement with our intuition that high coverage/sequence identity 544 alignments should be favored. For $P_{0}$, we use the fraction of interface residues in the train set (resp. 0.22 and 0.09 for ${ }_{545}$ the PPBS and BCE train sets). Note that since the labels are already defined using multiple pdb files and redundancy 546 reduction on templates was employed, there is no need to further reweight alignments by ligand diversity as described 547 in $[11]$.

${ }_{548}$ As expected, the baseline performed very well when high quality homologs were available, and underperformed 549 otherwise.

551 We used the Docker image of Masif-site as made available at https://github.com/LPDI-EPFL/masif. Masif-site ${ }_{552}$ predicts binding site propensity at the surface vertex level. To aggregate at the amino acid level, we followed the 553 aggregation scheme provided for the Masif vs Sppider comparison (https://github.com/LPDI-EPFL/masif/blob/ 554 master/comparison/masif_site/masif_vs_sppider/masif_sppider_Intpred_comp.ipynb): each surface vertex 555 is first assigned to its closest atom and corresponding amino acid and the binding site probability of an amino acid 556 is taken as the maximum binding site probability over all its corresponding vertices. We stress that the comparison 557 with Masif-site should be interpreted with caution, as:(i) Masif-site predicts at surface vertex level rather than amino 558 acid level. Its residue-wise probabilities are therefore not calibrated, resulting in bad likelihood scores (see Sup. Table 559 S2). (ii) We did not retrain Masif-site owing to limited computational resources and ts training set used was smaller 560 than ours (iii) Our test set overlaps with Masif-site training set, hence Masif-site should overperform on a fraction of 561 our test set. 
${ }_{563}$ We used the Discotope version 1.1 as made available at https://services.healthtech.dtu.dk/software.php. ${ }_{564}$ To emulate the behavior of Discotope version 2.0, which processes entire protein assemblies rather than individual ${ }_{565}$ protein chains [51], we fused each multi-chain antigens into a single chain, and verified on a few examples that the 566 outputs were consistent with the ones from the Discotope 2.0 webserver.

\section{Data preparation}

${ }_{568}$ Initial database and filtering We use the Dockground database of protein-protein interfaces [45] (Jan. 2020 full ${ }_{569}$ redundant version) as a starting point for our Protein-Protein Binding Sites (PPBS) database. Each unique PDB 570 chain involved in one interface or more was considered as a single example; we excluded chains with sequence length ${ }_{571}$ less than 10, chains involved in a protein-antibody complex (as classified in the SabDab database [50]), or designed ${ }_{572}$ proteins (identified as having two or more of the following red flags: no Uniprot ID, no known CATH class, no sequence 573 homologs found, and engineered/synthetic/designed/de novo appearing in chain name). We obtained 70583 unique 574 chains (grouped in 20025 clusters at 95\% sequence identity) from 41466 distinct PDB files, involved in 240506 PPIs. 575 The dataset covers a wide range of complex sizes, types, organism taxonomies, protein lengths (Fig.S4 (a)-(d)). For 576 the B-cell epitopes database, we used the SabDab database (timestamp: 04/19/2021 [50]) and included all antigens 577 with length 10 or more forming an interface with an antibody with both heavy and light chain appearing in the PDB 578 files. We obtained 3756 chains (grouped in 796 clusters at $95 \%$ sequence identity).

${ }_{579}$ Data partition For the PPBS database, we investigated the impact of homology between train and test set ${ }_{580}$ examples on generalization of ScanNet and our baseline models. We enforced a maximum sequence identity (90\%) 581 between a val/test example and any train set example and grouped validation and test examples into four subgroups 582 based on their degrees of homology, see Sup. Fig. S4(g):

1. Val/Test 70\%: At least $70 \%$ sequence identity with at least one train set example.

2. Val/Test Homology: At most $70 \%$ sequence identity with any train set example, at least one train set example belonging to same protein superfamily (H level of CATH classification [48]).

3. Val/Test Topology: At least one train set example with similar protein topology (T level of CATH classification [48]), none with similar protein superfamily.

4. Val/Test None: None of the above.

589 Subgroups are ordered by decreasing degree of homology; generalization is expected to be increasingly difficult. ${ }_{590}$ To ensure that the four subsets have approximately equal sizes, the following partitioning algorithm was employed. ${ }_{591}$ The chains are first iteratively clustered by sequence identity at several levels $(100 \%, 95 \%, 90 \%$ seqID, $70 \%$ seqID) 592 using CD-HIT [83] followed by clustering at homology and topology identifiers. If a 70\% (resp. homology) cluster ${ }_{593}$ contains several distinct homology (resp. topology) categories, these categories are merged into a single one. Next, 594 we constructed the $\mathrm{Val} /$ Test None by randomly drawing topology clusters and assigning all its members to either ${ }_{595}$ validation and test; this is repeated until Val/Test None are full. The Val/Test topology sets were constructed by 596 randomly drawing from the remaining topology clusters with more than one homology cluster, and assigning half of 597 the homology clusters to train and half to val/test. Similarly, the Val/Test homology and Val/Test 70 are constructed ${ }_{598}$ similarly by drawing homology (resp. 70\%) clusters with more than one $70 \%$ (resp. 90\%) sequence identity cluster, 599 and allocating each $70 \%$ (resp. 90\%) cluster to either train or val/test. Finally, the remaining $90 \%$ clusters are 600 randomly allocated to fill the training, validation and test sets (64\% - 16\% - 20\% split).

${ }_{601}$ For the BCE, the dataset was subdivided into 5 folds for cross-validation. Antigens were clustered at $70 \%$ sequence 602 identity, and each cluster was assigned to one fold at random (except for SARS-CoV-2 antigens, which were all 603 assigned to fold 1 ).

604 Label computation An amino acid of a protein chain is labeled as a binding site if at least one of its heavy atoms 605 is within $4 \AA$ of another heavy atom from another chain within the biological assembly [12]. Next, since the same 606 protein may appear in multiple assemblies, we take the union of all its binding sites found across pdb files. This is 607 done by clustering sequences at $95 \%$ sequence identity using CD-HIT [83, 84], aligning the sequences and labels of ${ }_{608}$ each cluster using MAFFT [85] and propagating the labels along each column. We found that for the PPBS dataset, ${ }_{609} 91.2 \%$ of the binding sites were identified from the original pdb complex file and $8.8 \%$ were propagated from other ${ }_{610}$ pdb files.

${ }_{611}$ For SabDab, we found that several epitopes appeared as accessible in one conformation of the protein and buried in ${ }_{612}$ another conformation; labels were propagated from one structure to another only if the residues had similar relative 
${ }_{613}$ accessible surface area and coordination number (number of amino acids within $13 \AA$ ). The propagation criterion 614 writes:

$$
\left|\mathrm{ASA}_{1}-\mathrm{ASA}_{2}\right| / \sigma(\mathrm{ASA})+\left|\operatorname{Coord}_{1}-\operatorname{Coord}_{2}\right| / \sigma(\text { Coord })<0.5
$$

${ }_{616}$ For the PPBS, we obtained $22.7 \%$ positive labels - 30\% when only considering the surface residues, with relative ${ }_{617}$ accessible surface $\geq 25 \%$ ( distributions shown in Sup. Fig.S4 (e,f) ). For the BCE, we found 8.9\% positive labels.

${ }_{618}$ Sample weighting and subsampling PDB covers unevenly the protein sequence space: many protein families do 619 not have any representative structure, whereas others such as immunoglobulins have tens of thousands. The sampling 620 is also biased within one family, as some genes and/or organisms are more frequently studied than others. To correct ${ }_{621}$ for the biases occurring at multiple scales, we apply the following hierarchical reweighting scheme:

$$
w=\frac{1}{\# \mathcal{C}_{100}} \times \frac{1}{\#\left\{\mathcal{C}_{100} \in \mathcal{C}_{95}\right\}} \times \frac{1}{\#\left\{\mathcal{C}_{95} \in \mathcal{C}_{90}\right\}} \times \frac{1}{\#\left\{\mathcal{C}_{90} \in \mathcal{C}_{70}\right\}}
$$

${ }_{623}$ Where $\mathcal{C}_{T}$ denotes the clusters at sequence identity cut-off $T$. This choice is such that each cluster at $70 \%$ sequence ${ }_{624}$ identity contributes a total weight 1 ; within each $70 \%$ cluster, each of the $K 90 \%$ clusters contributes a total weight ${ }_{625} 1 / K$, and so on. An example of set of weights is illustrated in Fig. S4 (h).

${ }_{626}$ In addition, this hierarchical choice ensures that the total weight of a cluster is invariant upon subsampling at some ${ }_{627}$ higher cluster identity level (e.g. the total weight of a $90 \%$ sequence identity cluster is invariant upon subsampling at ${ }_{628} 100 \%, 95 \%$ or $90 \%$ sequence identity). For the PPBS dataset, when hierarchical reweighting was used, we found no ${ }_{629}$ significant change of performance when training on the full set of chains or on $95 \%$ sequence identity representatives ${ }_{630}$ and therefore used the $95 \%$ sequence identity subset for speed gain purposes. When no reweighting or subsampling ${ }_{631}$ was used, performance significantly degraded, see Table I and Sup. Fig. S13. For the BCE database, the same ${ }_{632}$ approach was followed, without any subsampling - in order to include as many conformations as possible - and using ${ }_{633}$ a $90 \%$ sequence identity cut-off for the reweighting scheme, as similar proteins may have different epitopes.

\section{Impact of induced fit on ScanNet predictions}

${ }_{635}$ Protein structures undergo induced fit (i.e. conformational changes) upon binding. The magnitude of confor${ }_{636}$ mational changes varies, ranging from minimal rearrangement of side chain rotamers to extensive allosteric motion. ${ }_{637}$ ScanNet is mostly [86] trained on bound chains but applied to unbound ones. Owing to its high expressivity, it is a ${ }_{638}$ priori capable of picking up signature of bound conformations such as over-stretched side chains or unpacked helixes ${ }_{639}$ (see e.g. 4wwx:B of Sup. Fig. S12).

${ }_{640}$ We evaluated the predictive performance of ScanNet on unbound chains for two datasets: the Dockground simulated ${ }_{641}$ and Dockground X-Ray [45]. The Dockground simulated data set consists of chains extracted from complex pdb files ${ }_{642}$ and relaxed using Langevin Dynamics simulations [87]. Simulating separately the bound protein structures, without ${ }_{643}$ the interacting partner for a short time period $(1 \mathrm{~ns})$ relaxes the side-chain conformations of the interface residues ${ }_{644}$ and reliably approximates the unbound form of the protein if conformational changes are small $(<2 \AA$ RMSD). We ${ }_{645}$ considered only the proteins that appeared in our data set and excluded four tetramers, obtaining 6012 chains. We ${ }_{646}$ used as ground truth the binding site labels of the PPBS data set (18.5\% positive labels).

${ }_{647}$ The Dockground X-Ray consists of chains that are both crystallized alone and in complex with their partner. It ${ }_{648}$ features chains undergoing larger conformational changes than the simulated data set one. We selected $N=709$ ${ }_{649}$ (bound, unbound) pairs with at least $95 \%$ sequence identity between chains. As some complex component were ${ }_{650}$ multi-chains, there was no direct correspondence with our data set labels (which included inter-domain, intra-protein ${ }_{651}$ binding sites); instead, we used as ground truth labels the interface residues of the complex (6.6\% positive labels).

${ }_{652}$ For both data set, we computed ScanNet predictions separately for the bound and unbound structures, excluded ${ }_{653}$ residues that did not match between the bound and unbound structure and compared both predictions residue-wise. ${ }_{654}$ Results are reported in Supplementary Table S1 and Supplementary Fig. S5. We find a good agreement between bound ${ }_{655}$ and unbound predictions (Pearson correlations of $r=0.86, r=0.78$ for simulated and X-ray data sets respectively). ${ }_{656}$ A slight drop in accuracy between bound and unbound structures is found: from $88.3 \%$ to $86.6 \%$ for the simulated set ${ }_{657}$ and from $91.9 \%$ to $91.3 \%$ for the X-ray set. We conclude that ScanNet predictions are overall robust to conformational ${ }_{658}$ changes, although improvements could be obtained by training on unbound structures. 


\begin{tabular}{|c|c|c|c|c|}
\hline Data set & Accuracy & Likelihood & AUCPR & Precision at 50\% Recall \\
\hline Dockground simulated (bound) & 0.884 & -0.280 & 0.736 & 0.793 \\
Dockground simulated (unbound) & 0.866 & -0.318 & 0.662 & 0.687 \\
\hline Dockground crystal (bound) & 0.919 & -0.216 & 0.294 & 0.276 \\
Dockground crystal (unbound) & 0.912 & -0.234 & 0.238 & 0.225 \\
\hline
\end{tabular}

TABLE S1. Performance evaluation for prediction of Protein-protein binding sites in the unbound setting

\begin{tabular}{|c|c|c|c|c|c|}
\hline Algorithm & Test (70\%) & Test (Homology) & Test (Topology) & Test (None) & Test (All) \\
\hline Structural homology baseline & -0.241 & -0.312 & -0.407 & -0.427 & -0.358 \\
Masif-site & NA & NA & NA & NA & -0.571 \\
Handcrafted features baseline & -0.360 & -0.350 & -0.371 & -0.377 & -0.364 \\
ScanNet & -0.293 & -0.284 & -0.284 & -0.315 & -0.294 \\
\hline
\end{tabular}

TABLE S2. Performance evaluation for prediction of Protein-protein binding sites. Average likelihood per residue (higher is better) is shown for the four subsets of test and the entire test.

\begin{tabular}{|c|c|c|c|c|c|}
\hline Algorithm & Test (70\%) & Test (Homology) & Test (Topology) & Test (None) & Test (All) \\
\hline Structural homology baseline & 0.916 & 0.886 & 0.844 & 0.844 & 0.868 \\
Masif-site & NA & NA & NA & NA & 0.712 \\
Handcrafted features baseline & 0.843 & 0.852 & 0.841 & 0.841 & 0.845 \\
ScanNet & 0.876 & 0.882 & 0.879 & 0.868 & 0.877 \\
\hline
\end{tabular}

TABLE S3. Performance evaluation for prediction of Protein-protein binding sites. Accuracy is shown for the four subsets of test and the entire test.

\begin{tabular}{|c|c|c|}
\hline Algorithm & AUCPR & PPV L/10 \\
\hline Structural homology baseline & 0.147 & 0.216 \\
Handcrafted features baseline & 0.139 & 0.191 \\
Discotope [51] & 0.114 & 0.191 \\
ScanNet (transfer learning) & $\mathbf{0 . 1 7 8}$ & 0.273 \\
ScanNet (no finetuning) & 0.106 & 0.211 \\
ScanNet (scratch) & 0.150 & 0.221 \\
ScanNet (transfer learning, no evolutionary information) & 0.173 & $\mathbf{0 . 2 7 5}$ \\
\hline Null prediction & 0.089 & 0.089 \\
Solvent Accessibility baseline & 0.121 & 0.178 \\
\hline
\end{tabular}

TABLE S4. Predictive performance for B-cell conformational epitopes. Area under Precision Recall Curve (AUCPR) and Positive Predicted Value at L/10 are shown. Evaluation by 5-fold cross-validation on the SabDab database.

\begin{tabular}{|c|c|c|c|}
\hline Epitope & Antibody & PDB ID & Reference \\
\hline RBD-A & CC12.1 & 6xc2:HL & {$[88]$} \\
RBD-B & COVA2-39 & 7jmp:HL & {$[89]$} \\
RBD-C & CV07-270 & 6xkp:HL & {$[90]$} \\
RBD-D & REGN10987 & 6xdg:AC & {$[91]$} \\
S309 & CV38-142 & 7lm8:MN & {$[92]$} \\
CR3022 (cryptic) & COVA1-16 & 7jmw:HL & {$[93]$} \\
NTD & $2-51$ & 7l2c:CD & {$[94]$} \\
Stem & B6 & 7m53:HL & {$[54]$} \\
\hline
\end{tabular}

TABLE S5. List of SARS-CoV2 representative antibodies shown in Fig. 5 . Epitopes are classified following [52] 


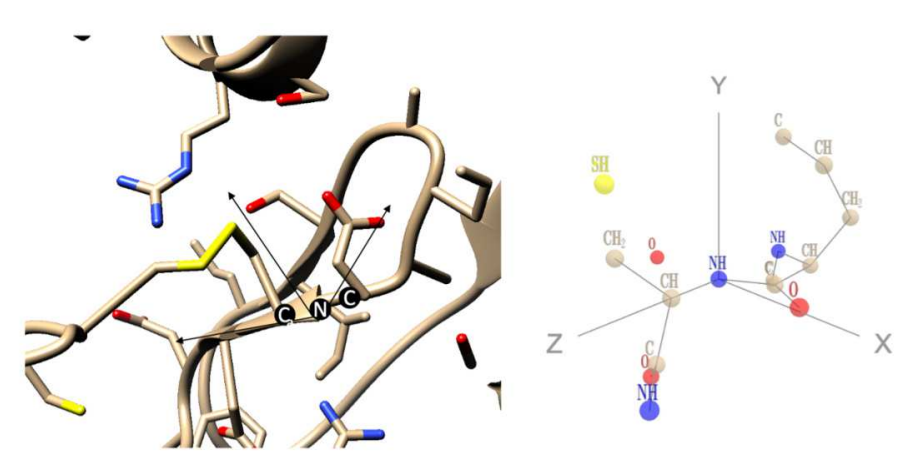

(a)

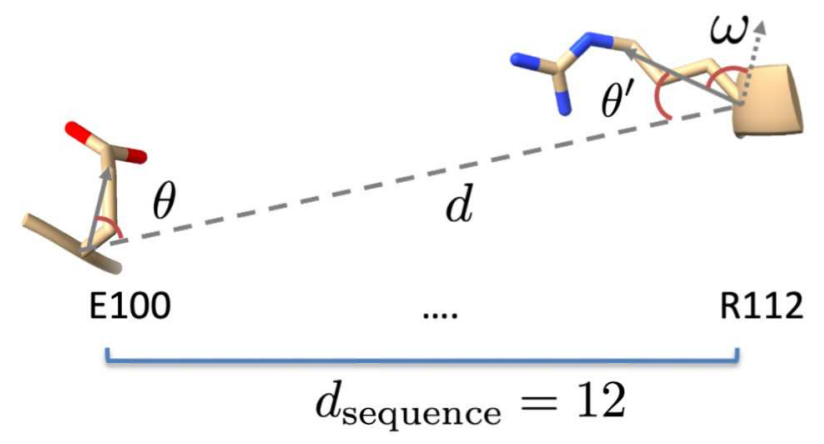

(c)

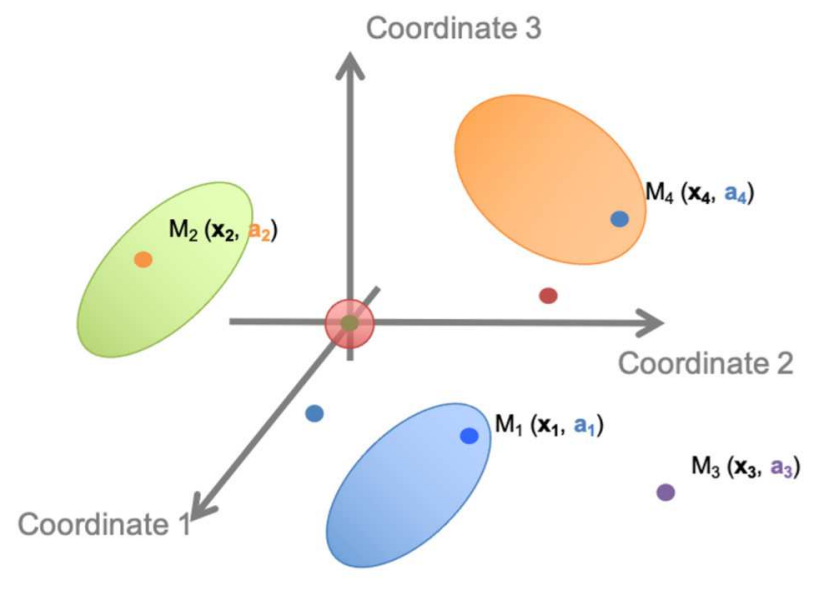

(d)
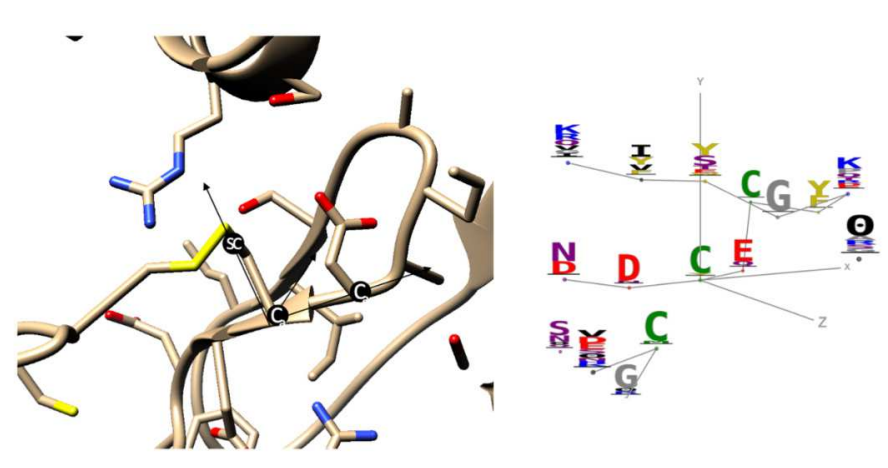

(b)
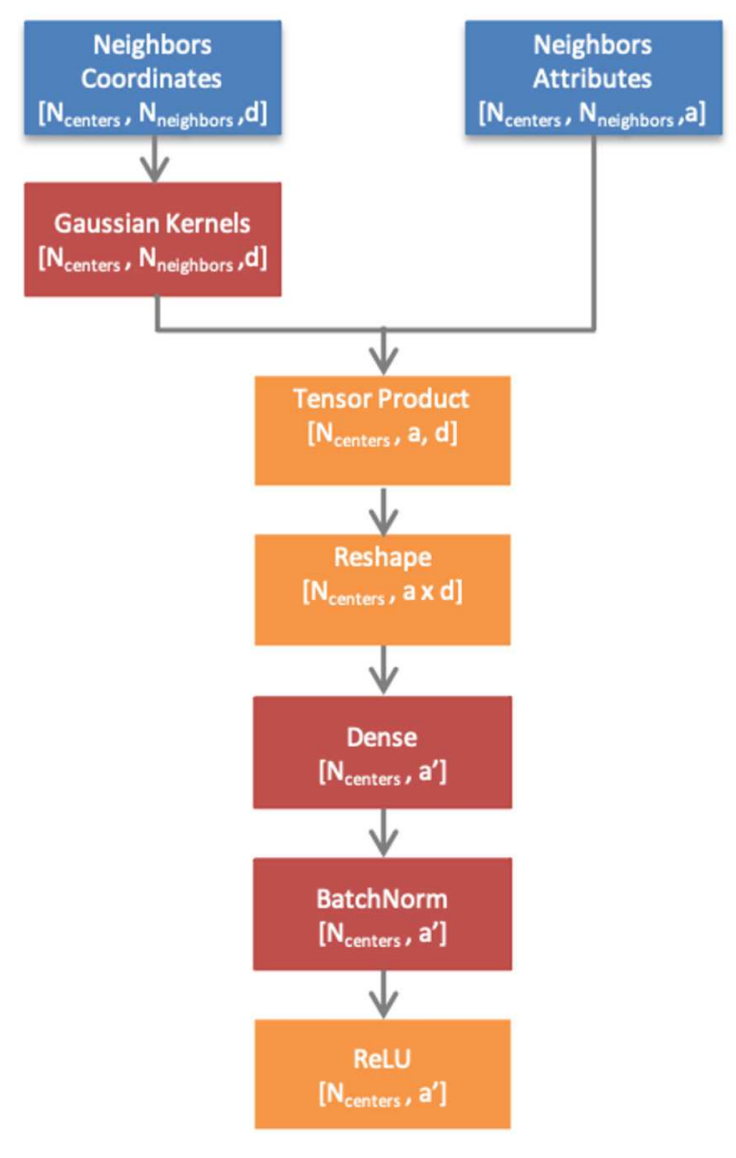

(e)

FIG. S1. Overview of the frame computation, neighborhood computation and neighborhood embedding modules (a) Construction of an atomic neighborhood from structure. For each atom, the $K=16$ closest atoms (including itself) are identified. Next, a frame is constructed from its position and the directions of its covalent bonds. The neighboring atoms are characterized by their coordinates in the local frame and group type (12 subclasses: $\mathrm{C}, \mathrm{CH}, \mathrm{CH} 2, \mathrm{CH} 3, \mathrm{CH}$ (aromatic ring), $\mathrm{O}, \mathrm{OH}, \mathrm{N}, \mathrm{NH}, \mathrm{NH} 2, \mathrm{~S}, \mathrm{SH}$. (b) Construction of an amino acid neighborhood from structure. For each amino acid, the $K=16$ closest amino acid (including itself) are identified. Next, a frame is constructed from its $\mathrm{C}_{\alpha}$ atom, side-chain center of mass and the previous $\mathrm{C}_{\alpha}$ atom along the backbone. The neighboring amino acid are represented by their coordinates in the local frame and their attributes learnt from the position weight matrix and pooled atomic filters. (c) Local coordinate system used for the neighborhood attention module (d) Principle of neighborhood embedding module: a generic neighborhood consists of a set of $K$ points $M_{k}$ characterized by their local coordinates $\mathbf{x}_{\mathbf{k}}$ and attributes $\mathbf{a}_{\mathbf{k}}$; (e) Implementation of the neighborhood embedding module. 


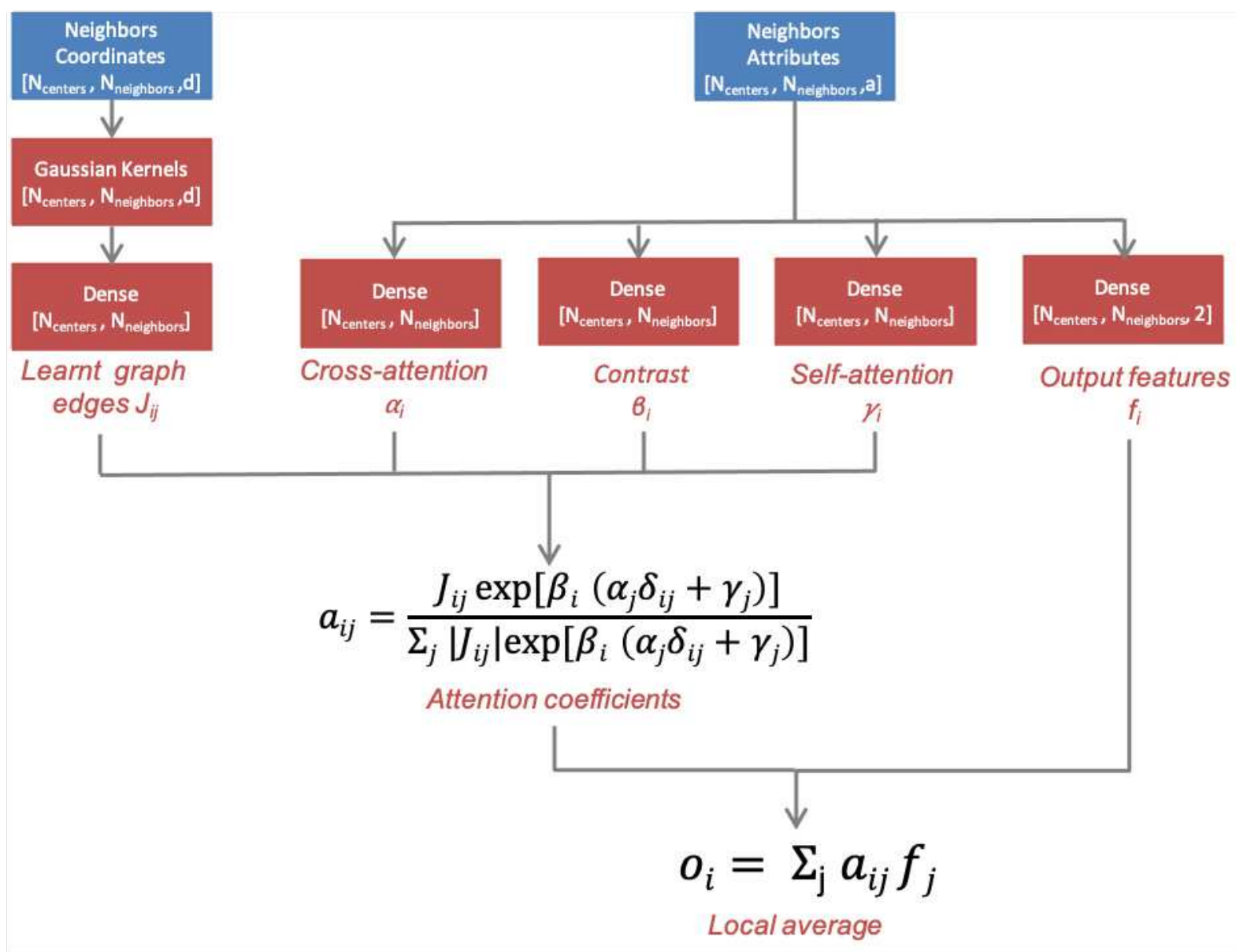

FIG. S2. Overview of the neighborhood attention module The neighborhood attention module is the final module of ScanNet; its purpose is to locally average predictions to produce spatially consistent predictions. An attention mechanism is included to account for driver/passenger binding sites. $\delta_{i, j}=1$ if $i=j ; 0$ otherwise is the Kronecker symbol. 


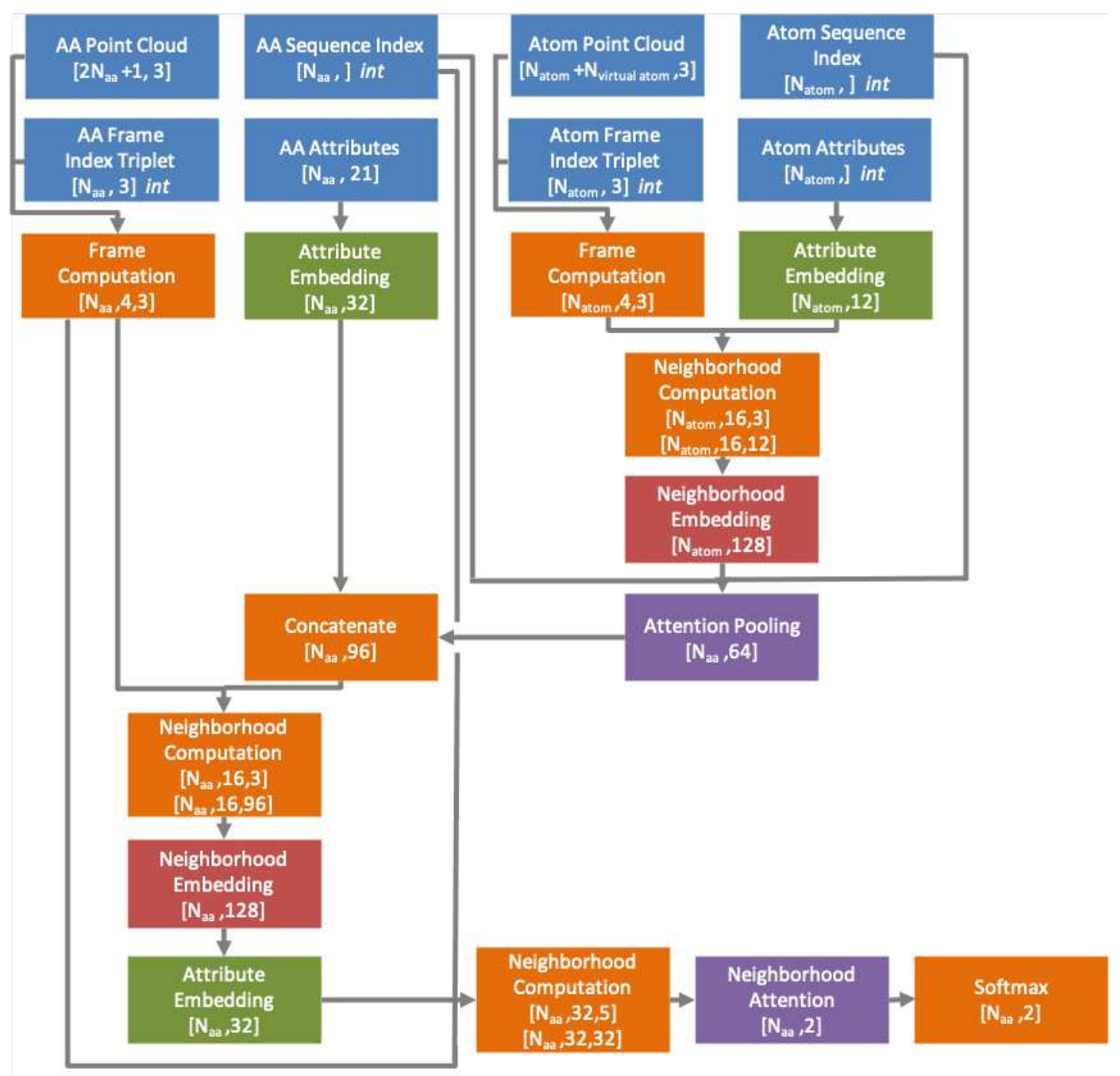

FIG. S3. Complete architecture of ScanNet Orange modules are not trainable. 


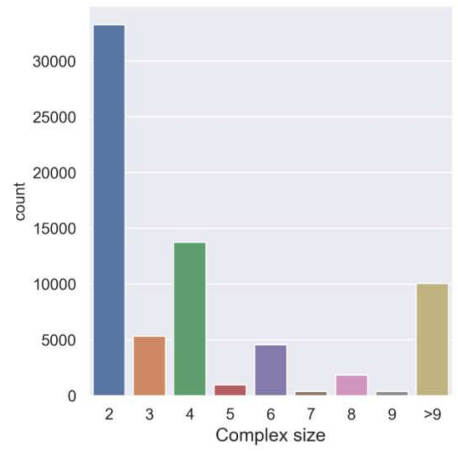

(a)

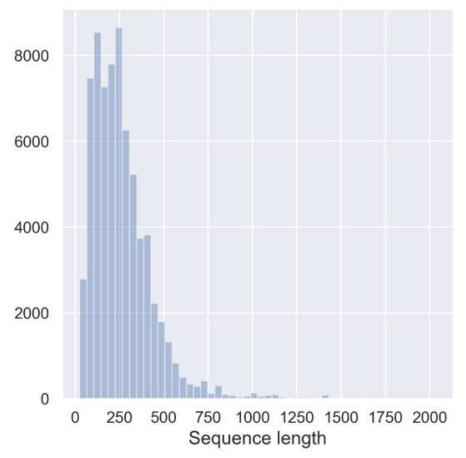

(d)

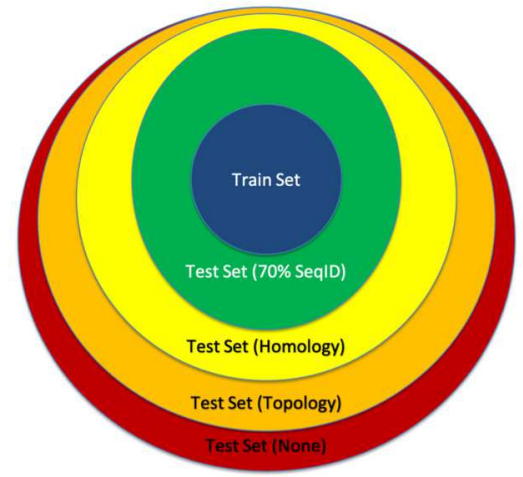

(g)

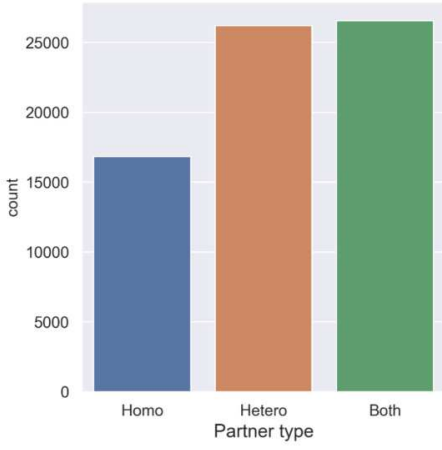

(b)

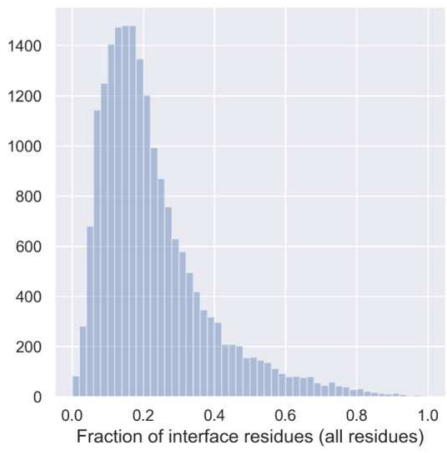

(e)

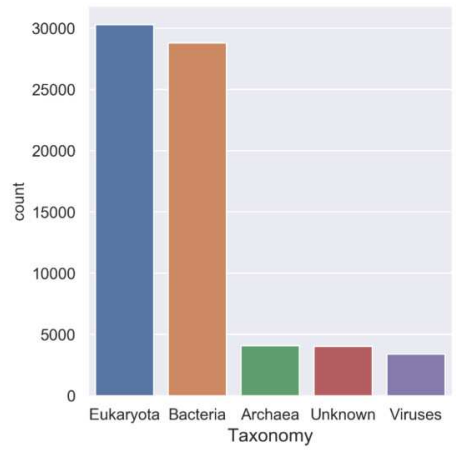

(c)

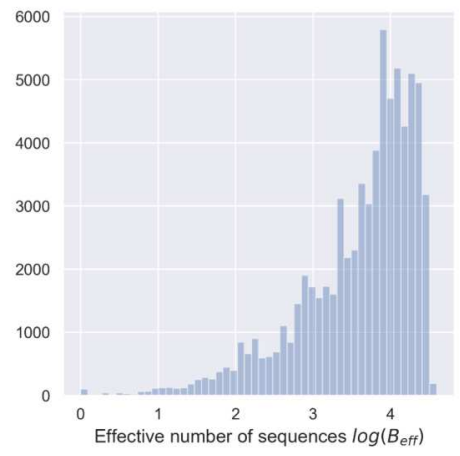

(f)

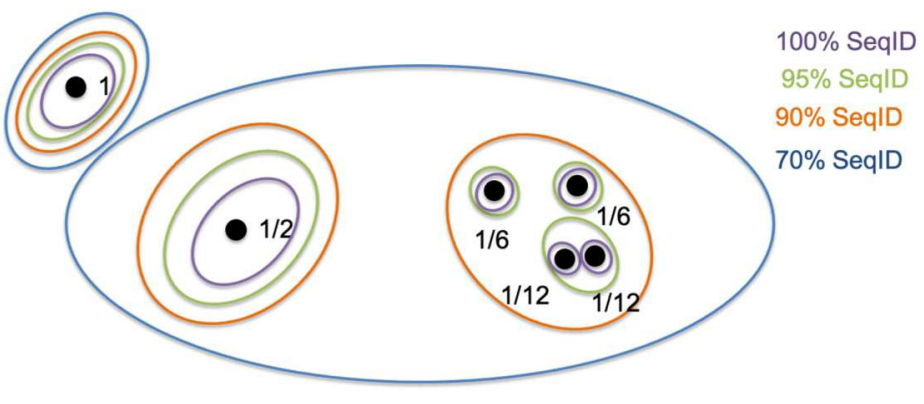

(h)

FIG. S4. Overview of the Protein-protein binding sites database (a-) Distribution of (a) complex sizes (b) complex types (c) source organism taxonomy (d) protein length (e) fraction of interface residues (f) effective number of sequences in corresponding the multiple sequence alignment. (g) Data partition. Proteins of the validation/test set are subdivided into four non-overlapping groups, depending on the degree of similarity with the closest protein found in the train set: (i) $\geq$ 70\% Sequence identity (ii) Same CATH superfamily (iii) Same fold topology CAT. (iv) None of the above. Generalization is increasingly difficult. (h) Illustration of the hierarchical sample reweighting used to counterbalance heterogeneity in the sampling of the protein space at multiple levels. Sequences are first clustered at four sequence identity thresholds (100\%, $95 \%, 90 \%, 70 \%$ ). Each cluster at $70 \%$ sequence identity (blue ellipses) contributes an identical total weight of 1 irrespective of its size. Within each $70 \%$ cluster, each of the $90 \%$ clusters (orange ellipses) contributes an identical total weight $1 /$ Ncluster90, etc. The weight of a sample is: Num(sequences in cluster 100) $\times$ Num(cluster 100 in cluster 95) $\times$ Num(cluster 95 in cluster $90) \times$ Num(cluster 90 in cluster 70$)$. The weight of each cluster $70 \%$ is invariant upon subsampling of the dataset. 


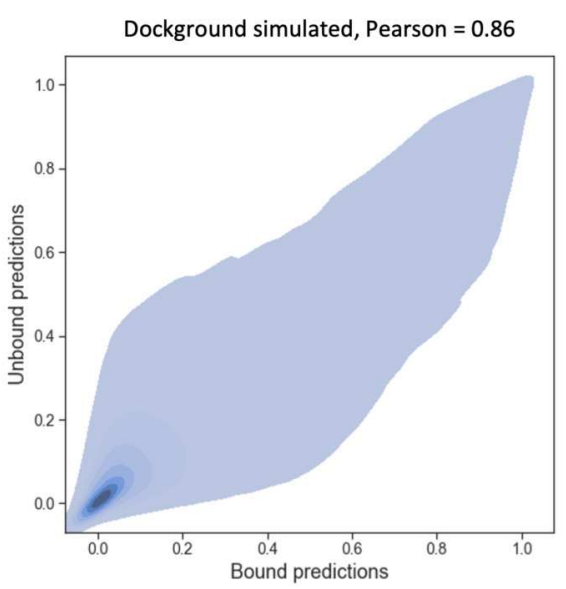

(a)

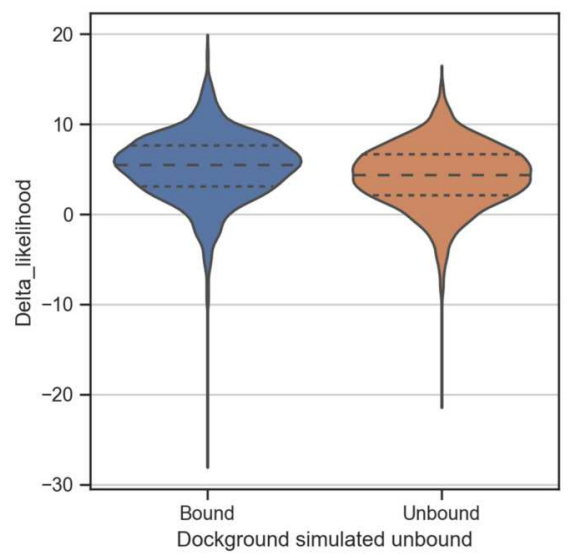

(c)

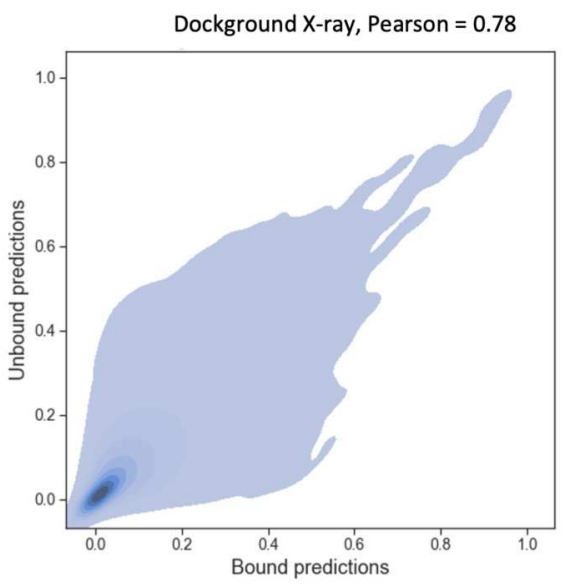

(b)

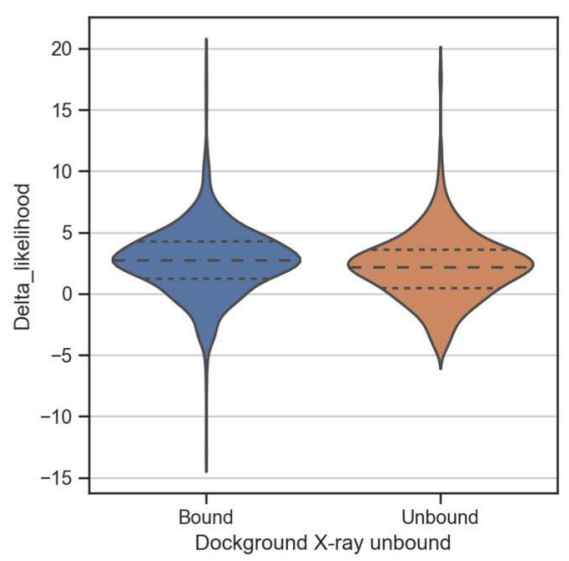

(d)

FIG. S5. Comparison between predictions performed on bound and unbound structures Two data sets of (bound,unbound) pairs of protein structures are considered: the Dockground simulated data set and Dockground X-ray data set. Panels (a),(b) display 2D-density plots of the distribution of ScanNet predictions on bound and unbound structures for each data set. Panels (c),(d) show for each data set the distributions of protein-wise prediction performance, measured as the difference $\Delta \mathcal{L}$ between the likelihood of ScanNet prediction and null prediction (uniform probability $p \sim 0.2$ ), divided by the standard deviation of the null model likelihood $\left(\sqrt{L p(1-p)} \log \left[\frac{p}{1-p}\right]\right)$. Higher is better. By construction, for a null predictor, $\Delta \mathcal{L}$ has zero variance across the data set whereas other metrics such as likelihood or accuracy have substantial variance owing to the variability of fraction interface residues across proteins, see Sup. Figure S4 (g); using $\Delta \mathcal{L}$ therefore facilitates detection of trends. A statistically significant but overall limited drop in performance is observed from bound to unbound. 


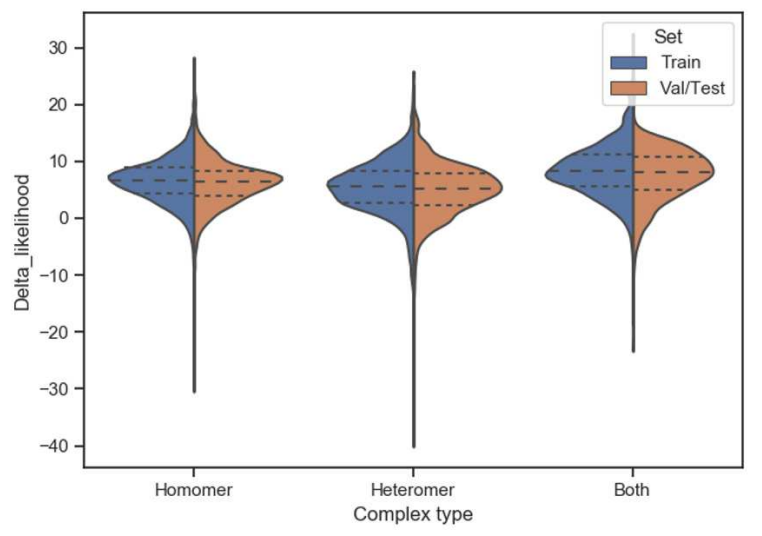

(a)

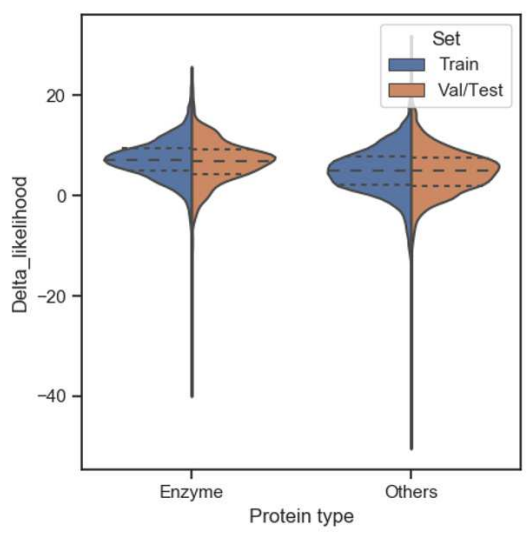

(b)

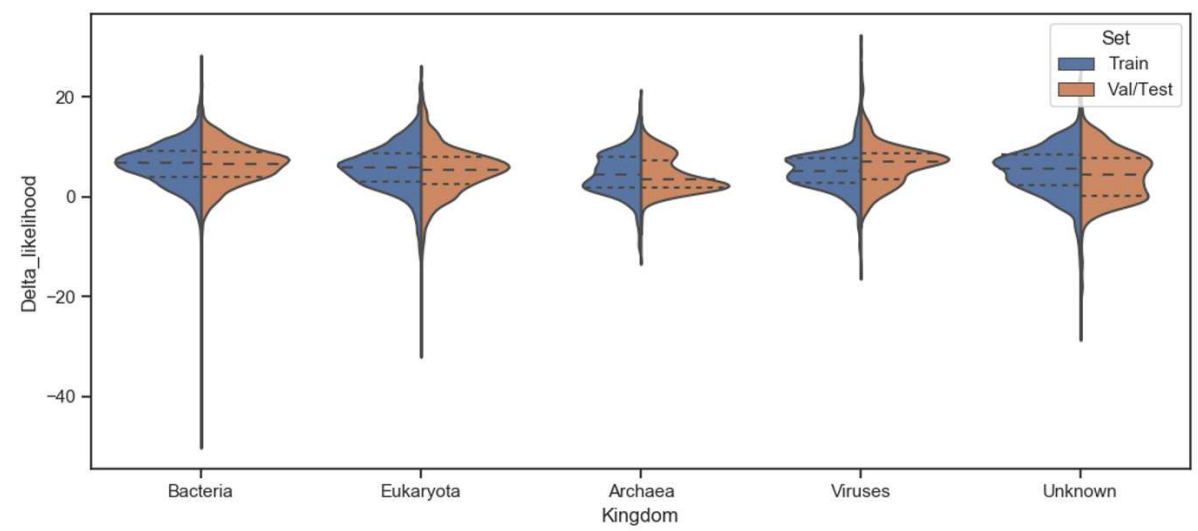

(c)

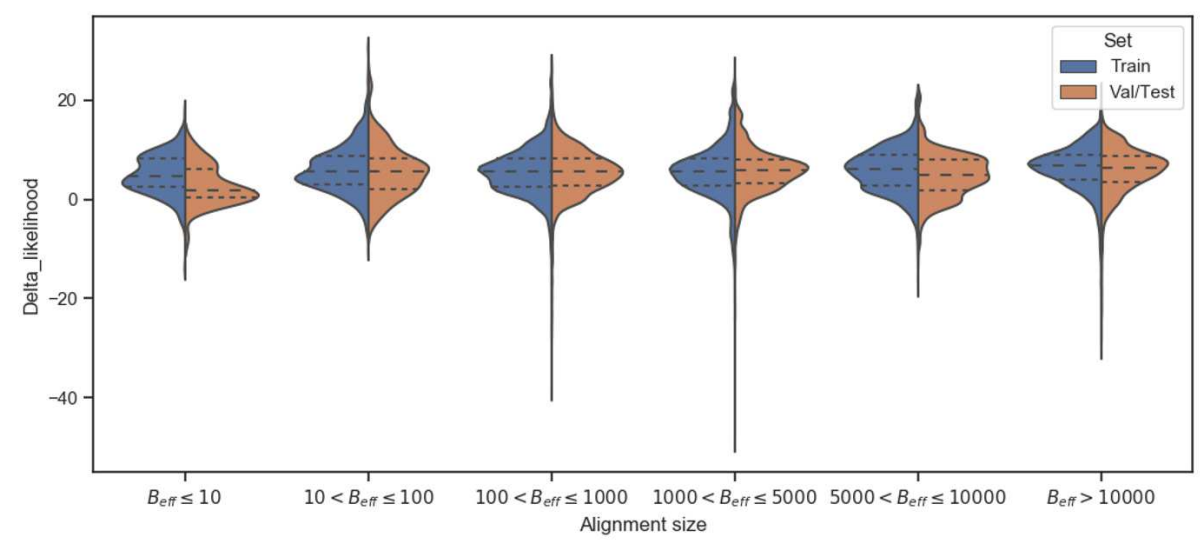

(d)

FIG. S6. ScanNet performance by sample type The metric shown is the difference between the likelihood of the ScanNet and the likelihood of the null predictor (constant probability $\sim 0.2$ ); higher is better. Prediction performance is shown against complex type, protein type, source organism and effective alignment size. 

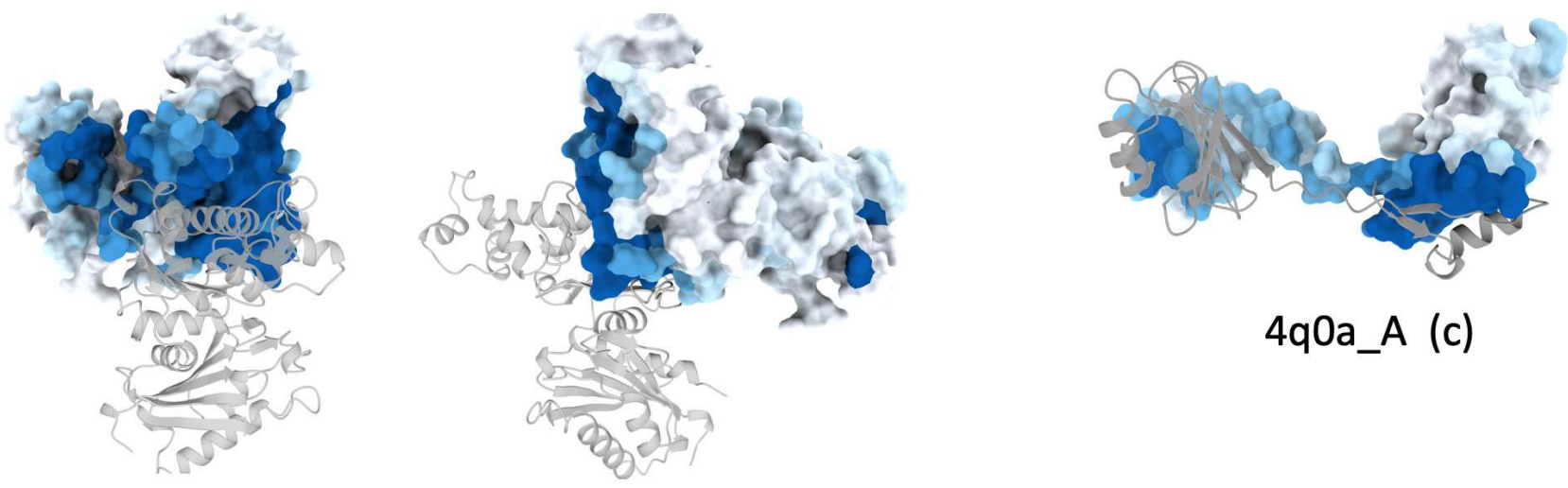

4q0a_A (c)

2qm1_B (a)

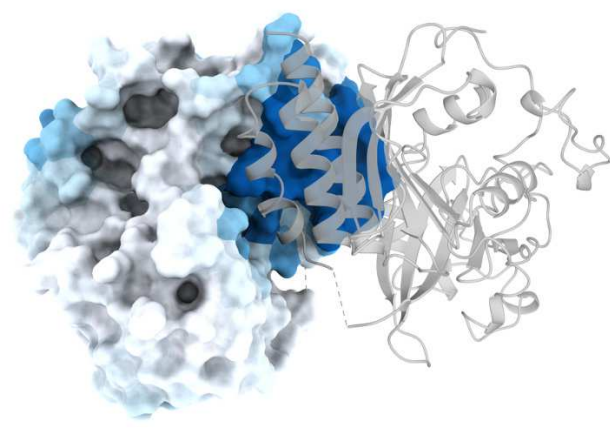

4q0h_A (b)

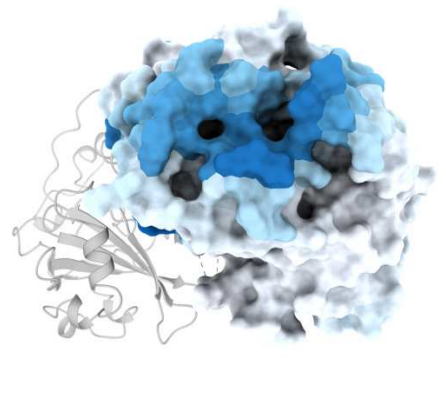

(b)

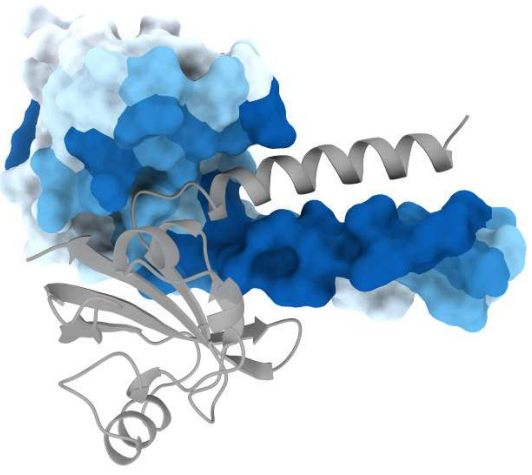

3bwl_A (d)

FIG. S7. Visualization of ScanNet PPBS prediction for homodimers. Typical test sets examples are shown, with delta likelihoood values close to the median performance of the test set. 

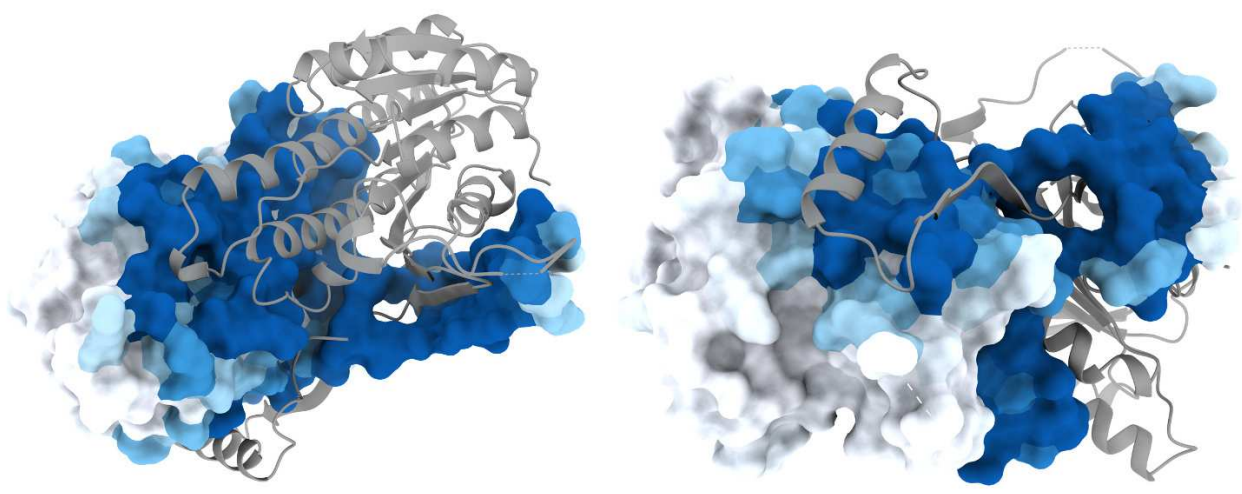

4kzp_D (a)

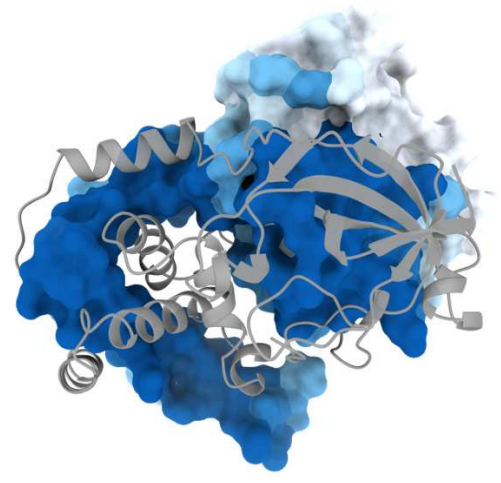

\section{1ugs_A (b)}

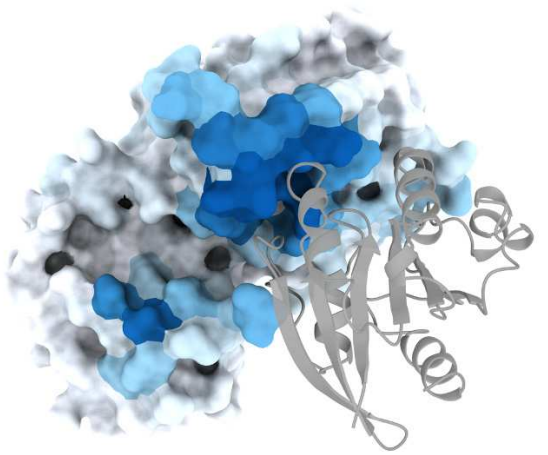

3bh6_B (c)

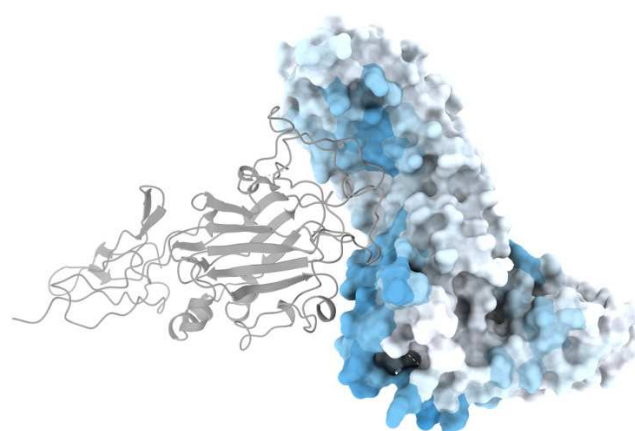

3zyj_A (d)

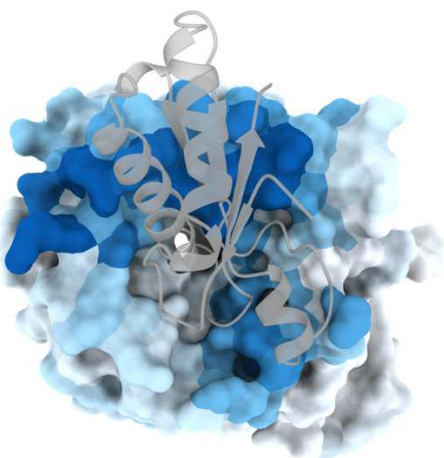

2hqs_A (e)

FIG. S8. Visualization of ScanNet PPBS prediction for heterodimers. Typical test sets examples are shown, with delta likelihoood values close to the median performance of the test set. 

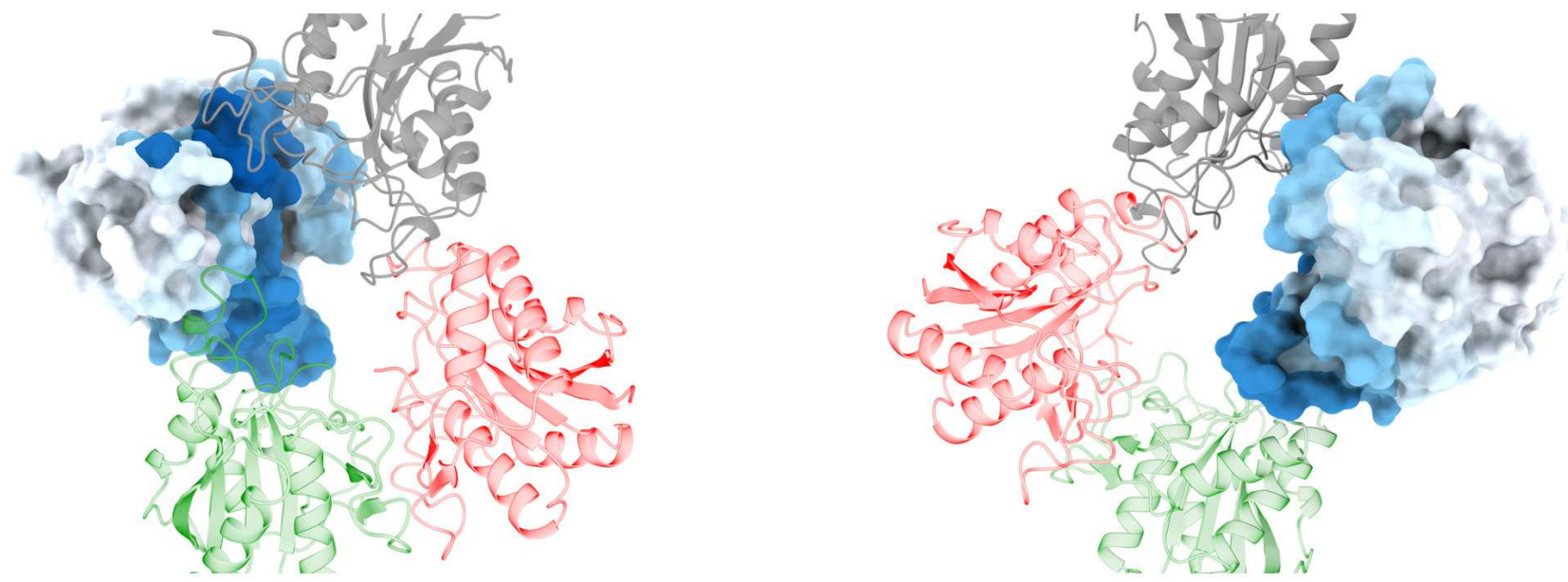

1aug_A (a)
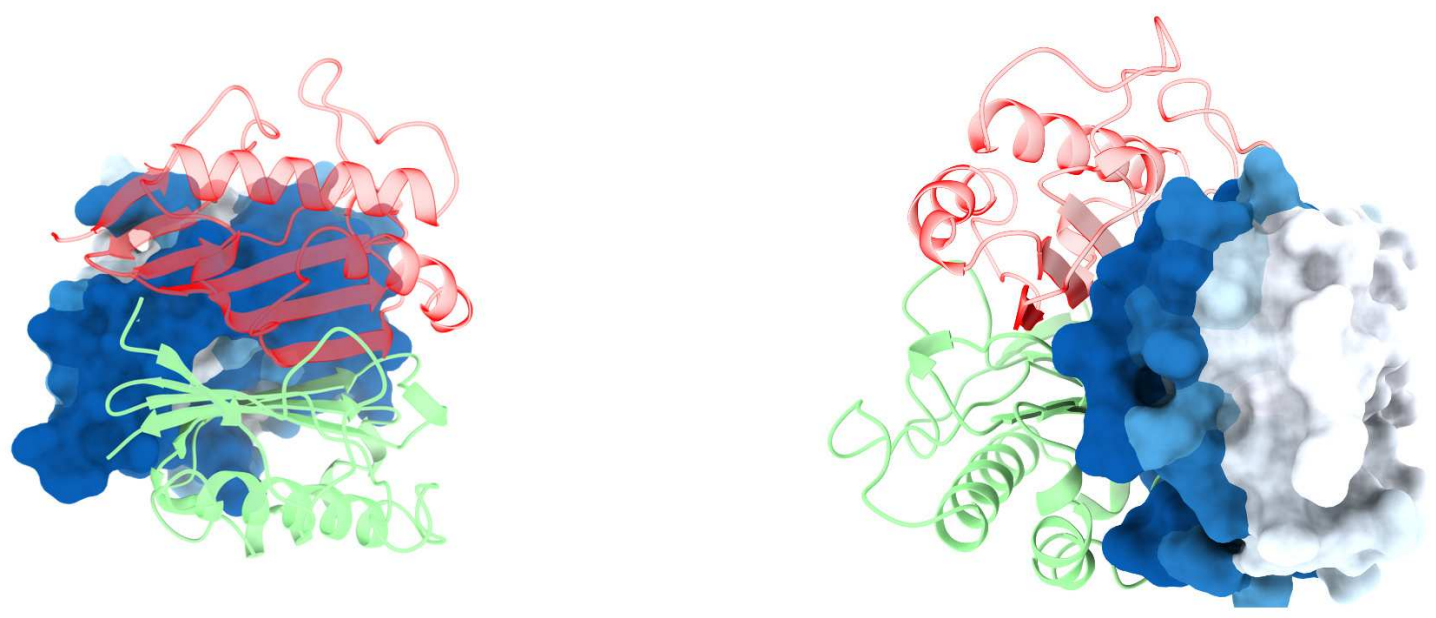

1x25_A (b)

FIG. S9. Visualization of ScanNet PPBS prediction for homomultimers. Typical test sets examples are shown, with delta likelihoood values close to the median performance of the test set. 

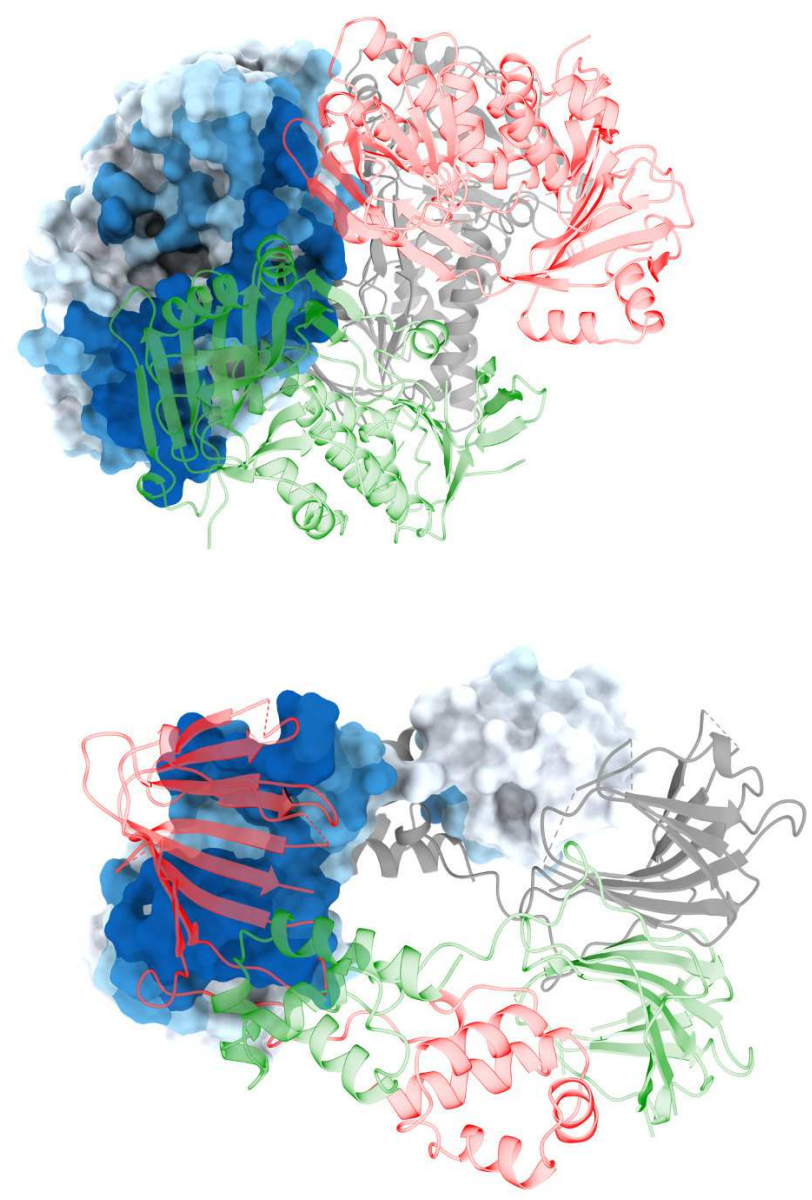

1zz6_A

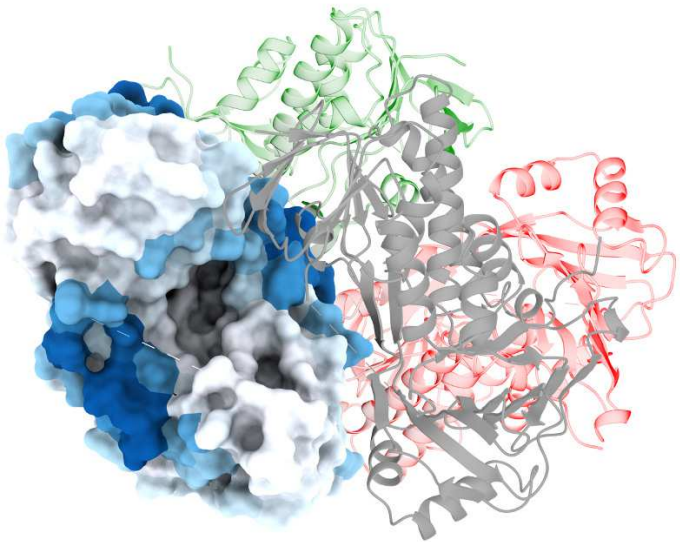

4k22_A

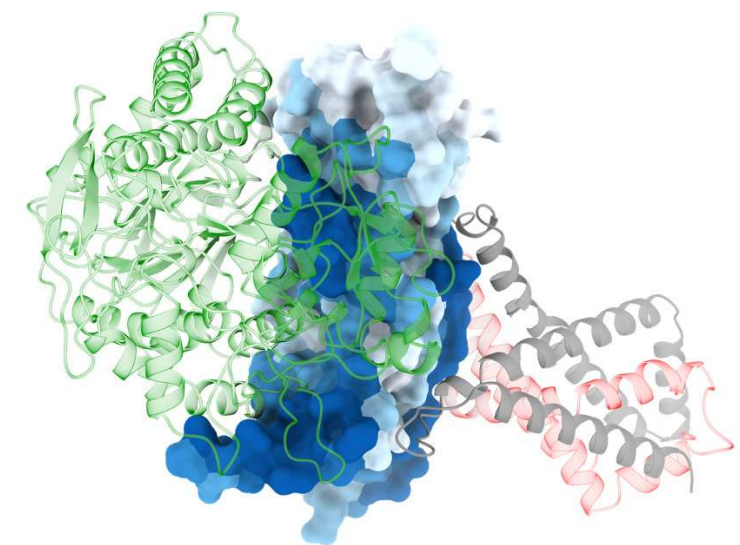

4k22_A

FIG. S10. Visualization of ScanNet PPBS prediction for heteromultimers. Typical test sets examples are shown, with delta likelihoood values close to the median performance of the test set. 


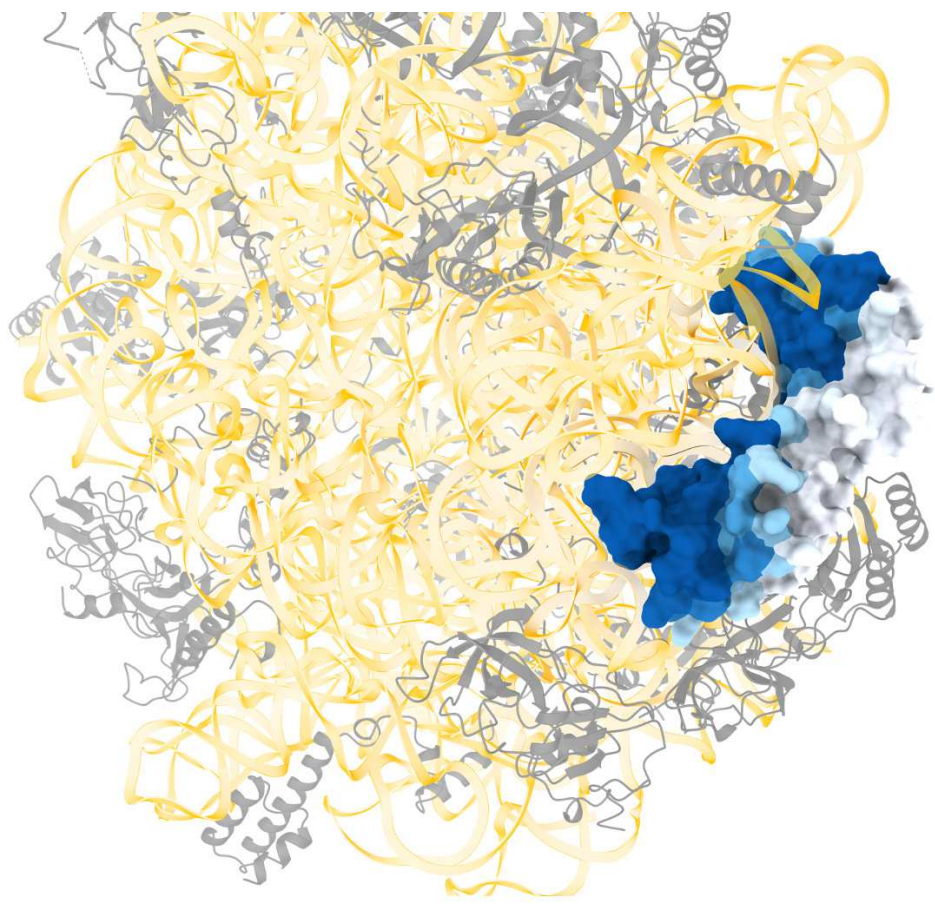

1jj2_E

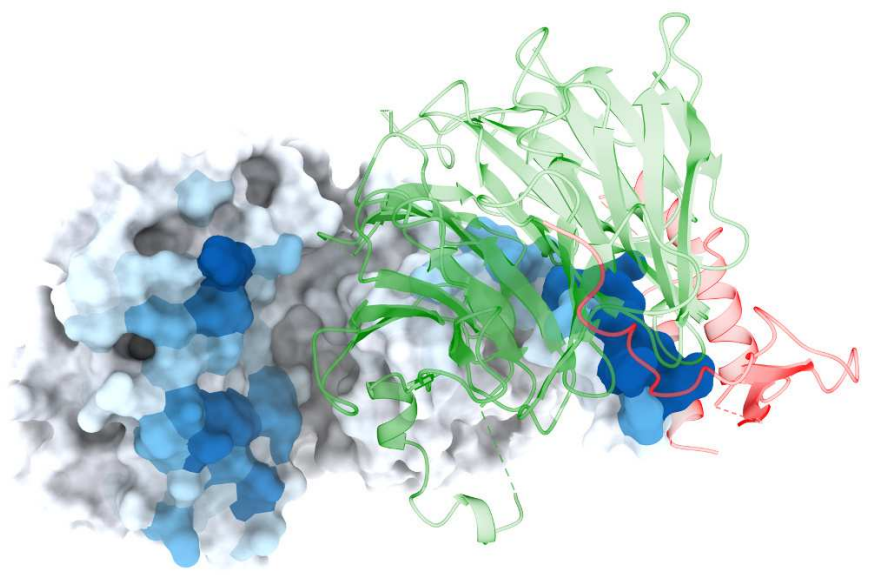

3w15_C

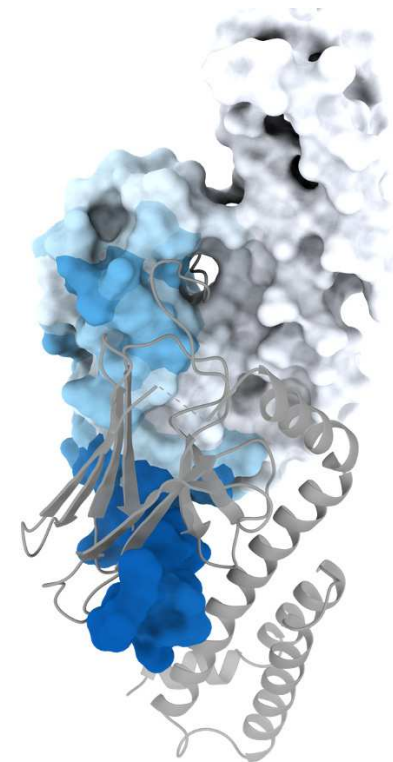

4r5I_B

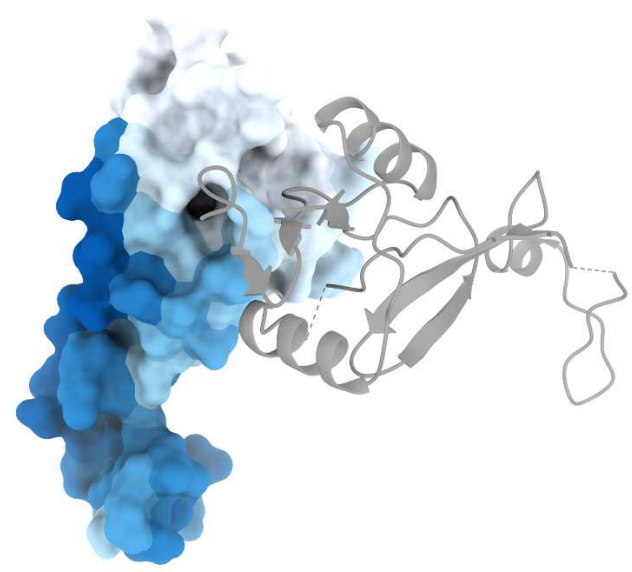

3t3a_A

FIG. S11. Example of incorrect PPBS predictions. Examples shown have delta likelihoood values in the lower decile of the test set. In the first instance, the network confuses an RNA binding site with a protein-protein binding site. In the second instance, the network fails to identify the cavity as a binding site for the histidine tag of the protein partner. The last two show misplaced binding sites 


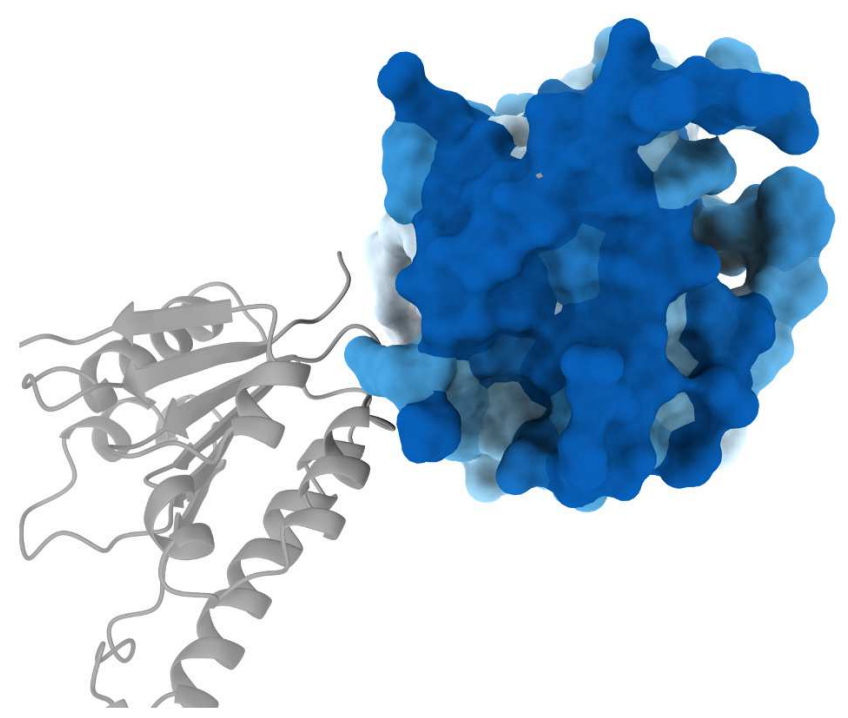

1g5r_A (biounit1)

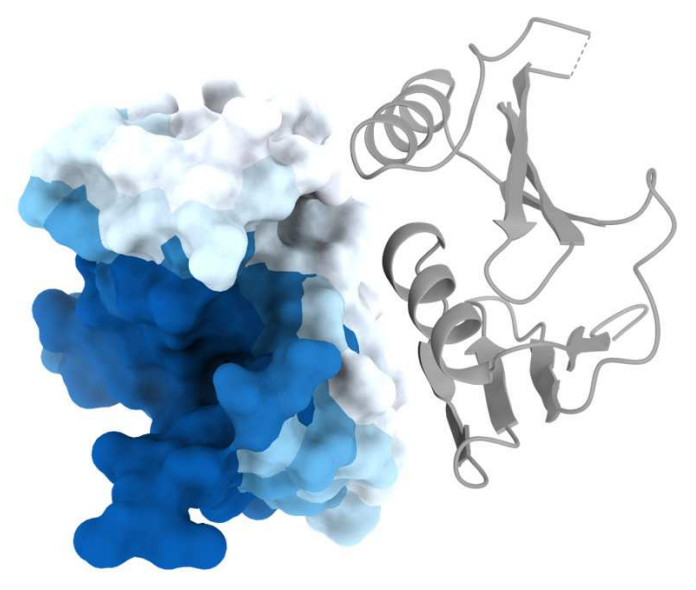

1xqa_A (biounit 1)

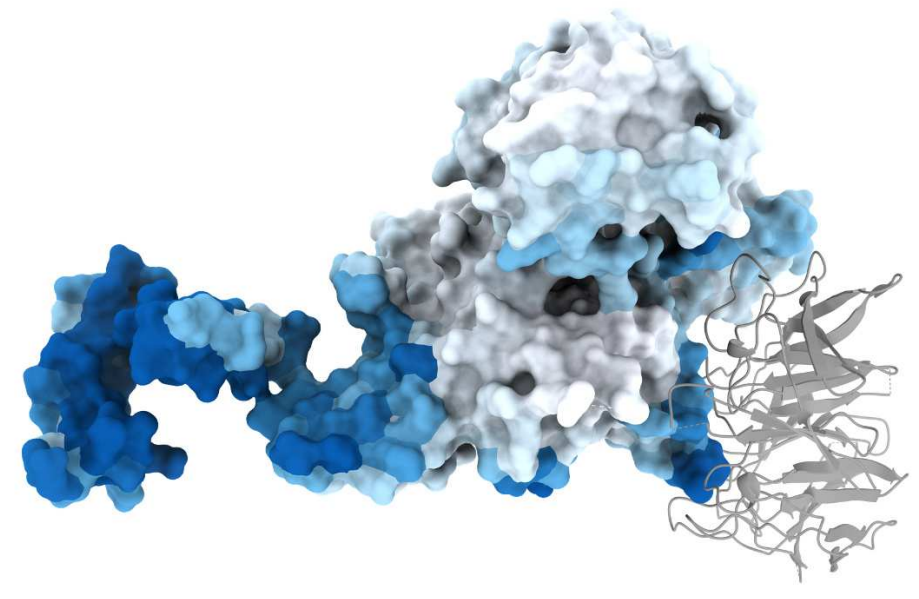

4wwx_B (biounit 1)

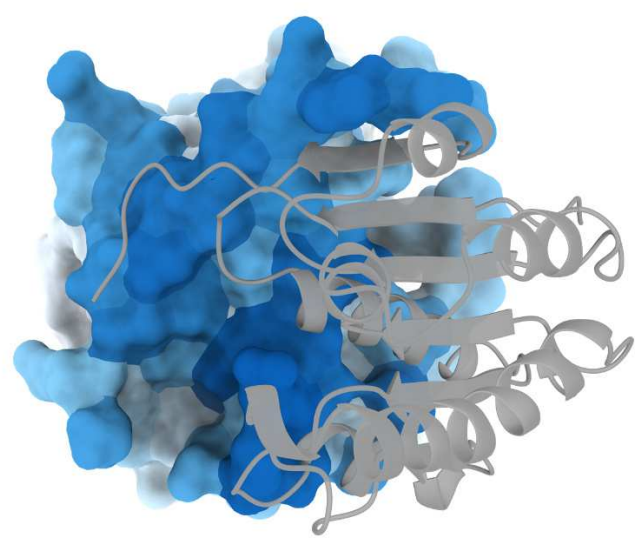

1g5r_A (biounit2)

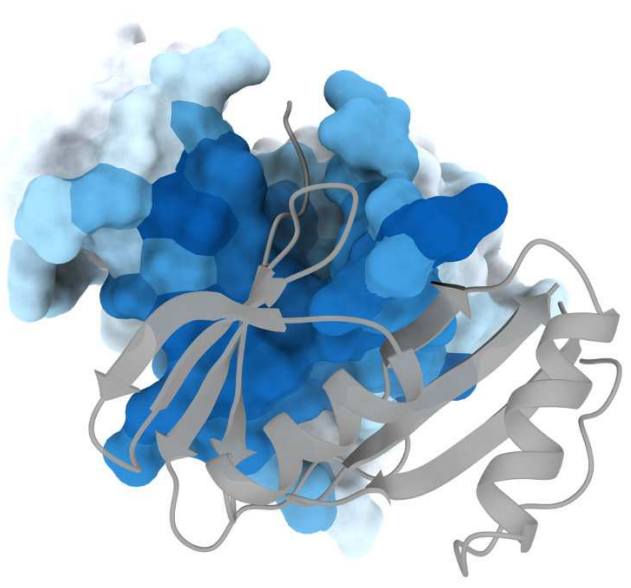

1xqa_A (biounit 2)

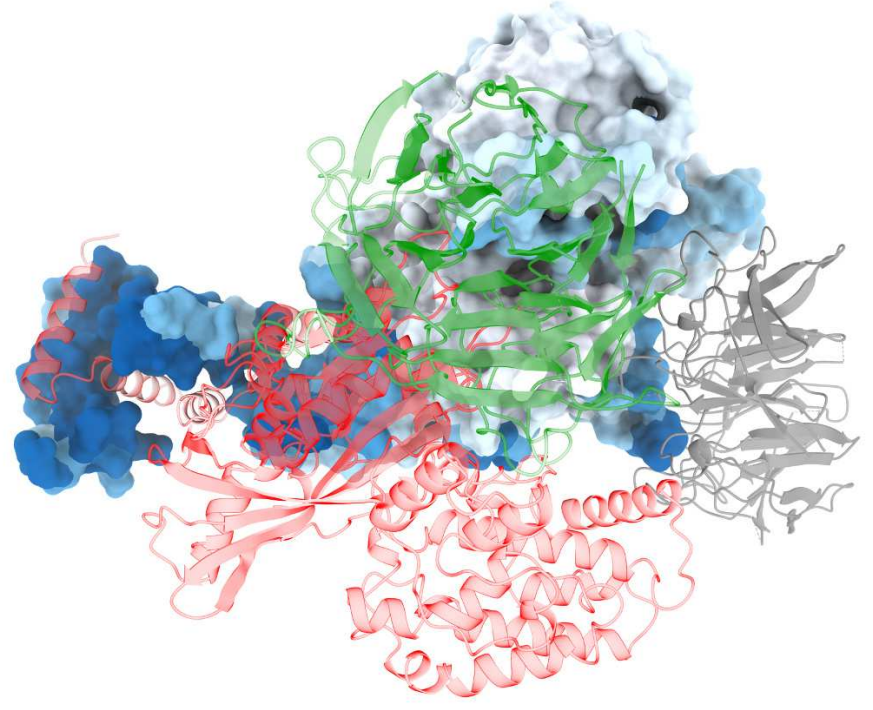

$4 w w x \_B(p d b)$ 


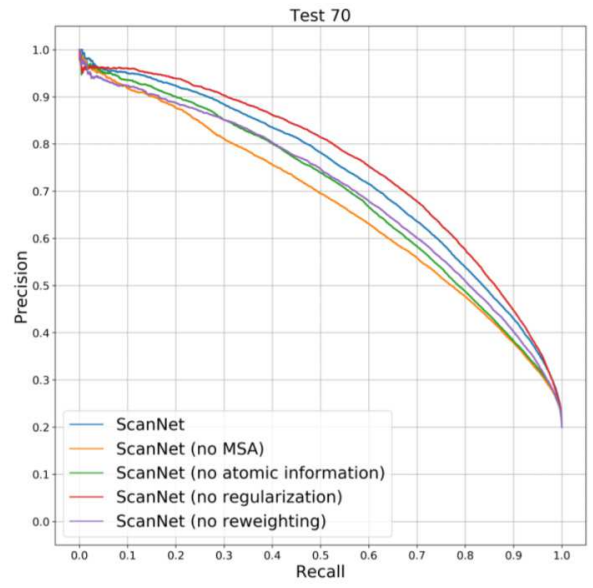

(a)

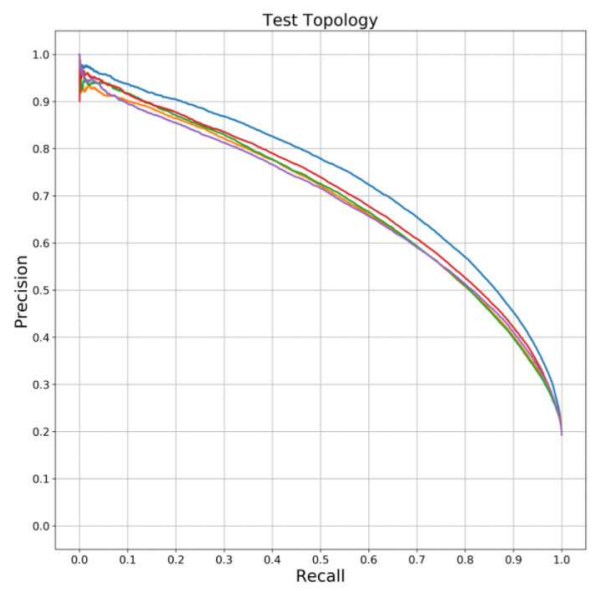

(c)

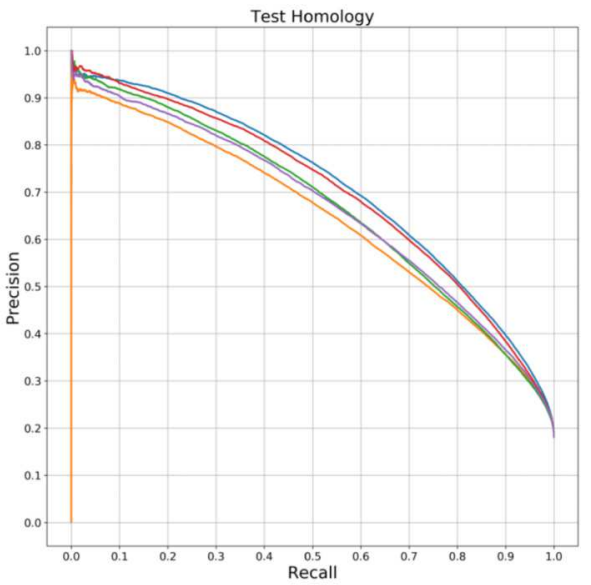

(b)

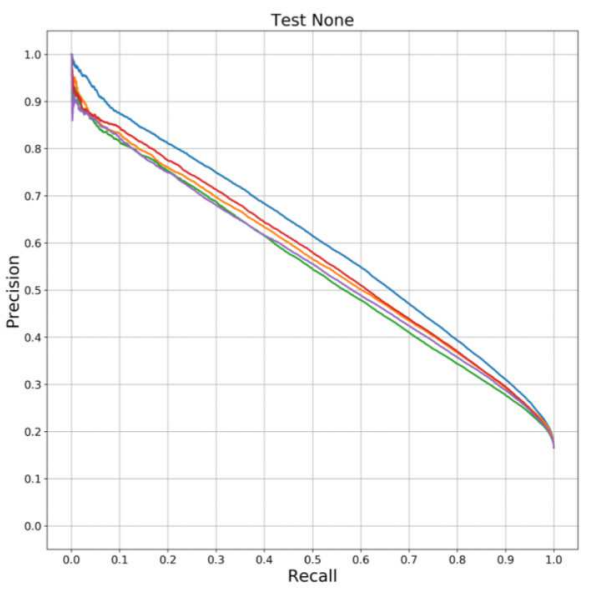

(d)

FIG. S13. Performance of Protein-Protein Binding Sites (PPBS) prediction with ablated ScanNet, see description of ablations in main text. Precision-Recall curves on the train set and the four test subsets, see Fig. 2. 


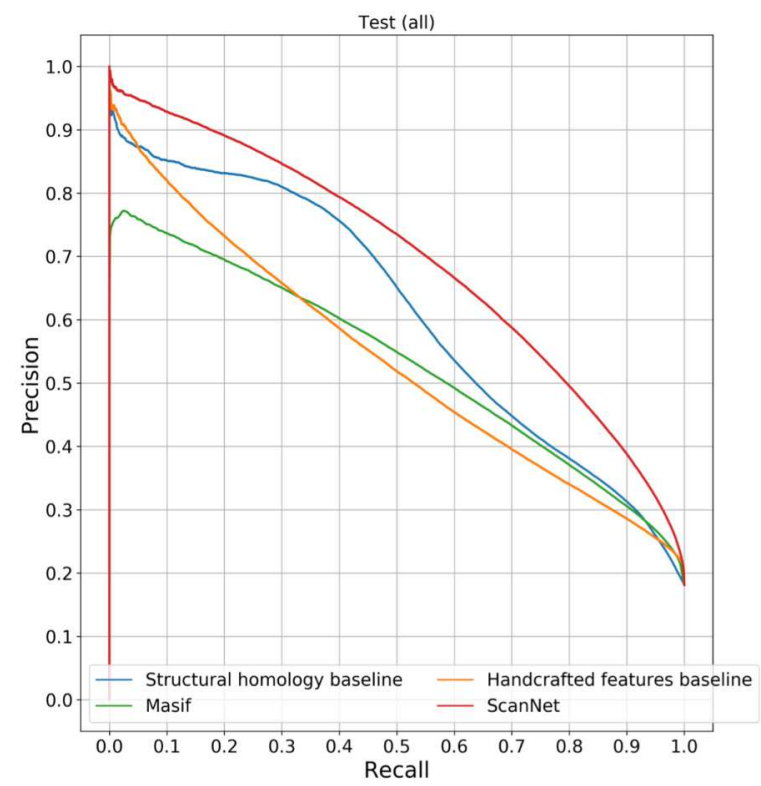

(a)

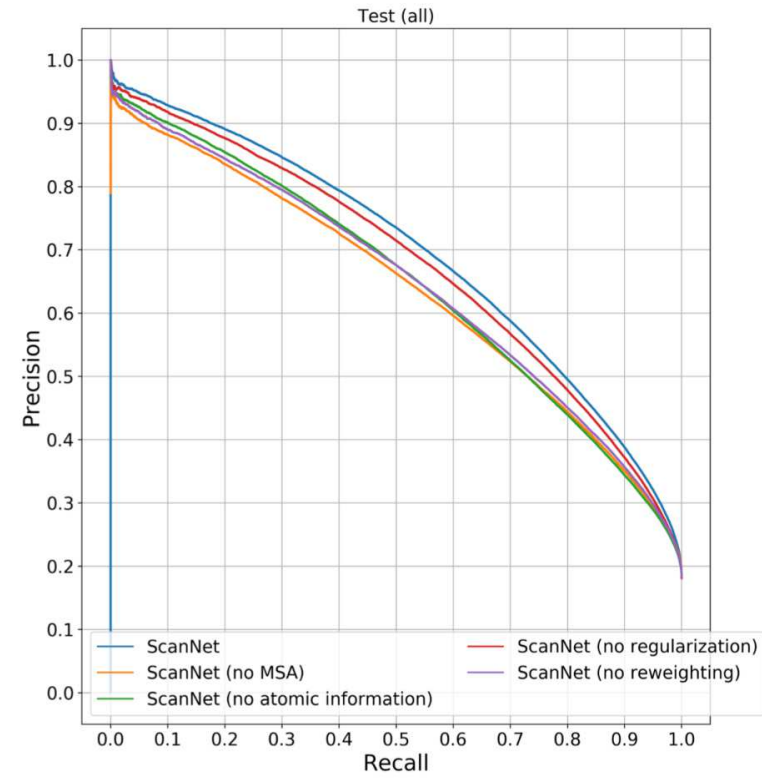

(b)

FIG. S14. Performance of Protein-Protein Binding Sites (PPBS) prediction Precision-Recall curves of PPBS prediction performance, across the entire test set. 


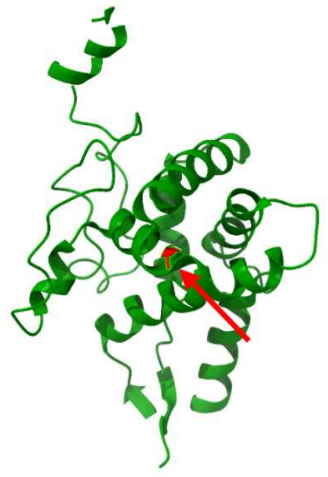

4v88/AF:98@N

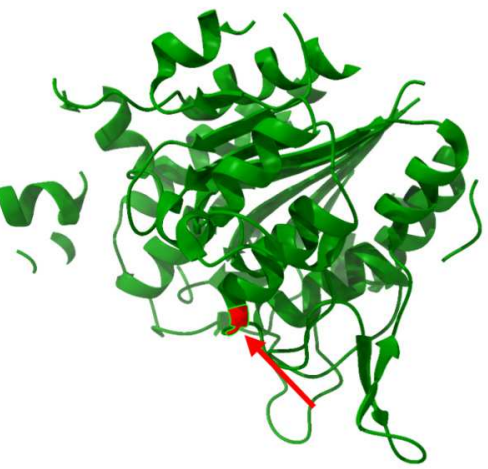

2x98/A:185@N

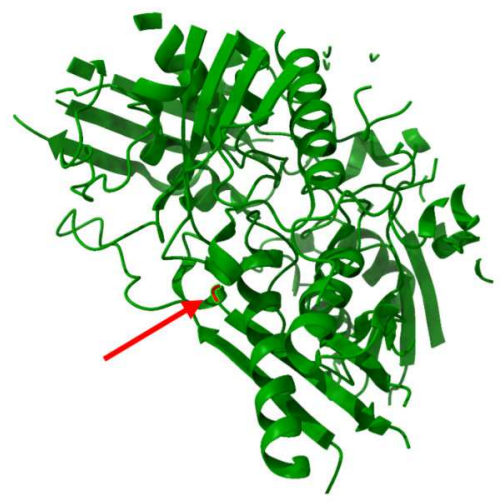

3ax7/A:1015@N

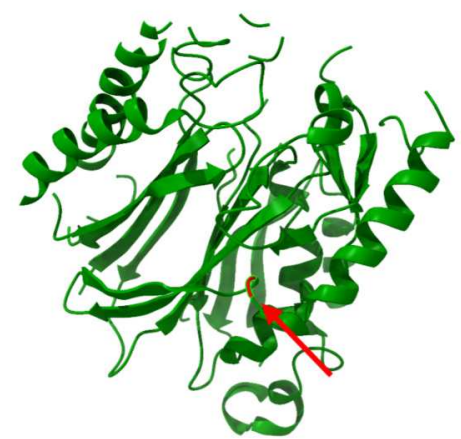

1umg/A:312@N

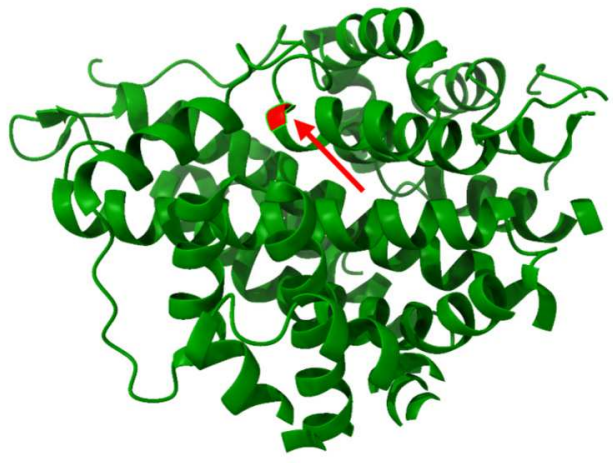

3u33/A:25@N

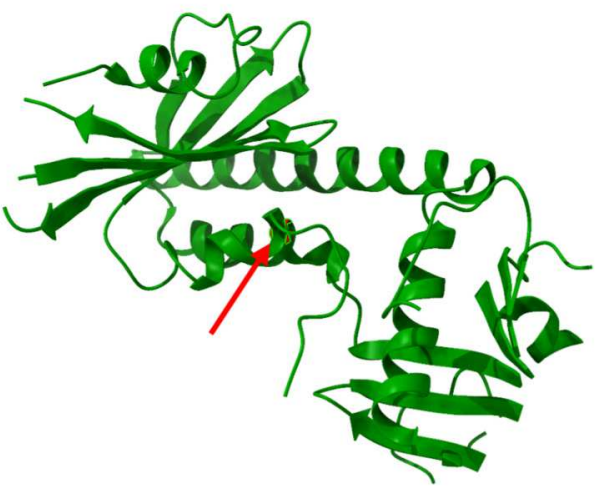

1srq/A:185@N

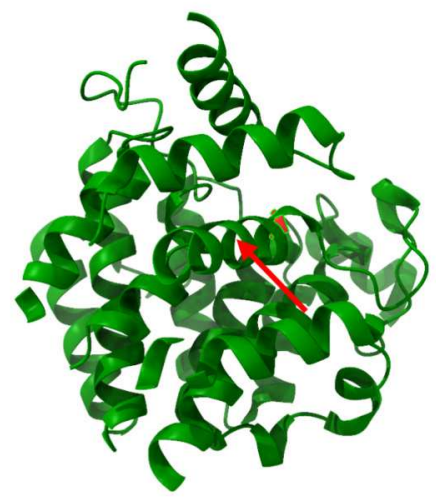

3o5t/A:243@N

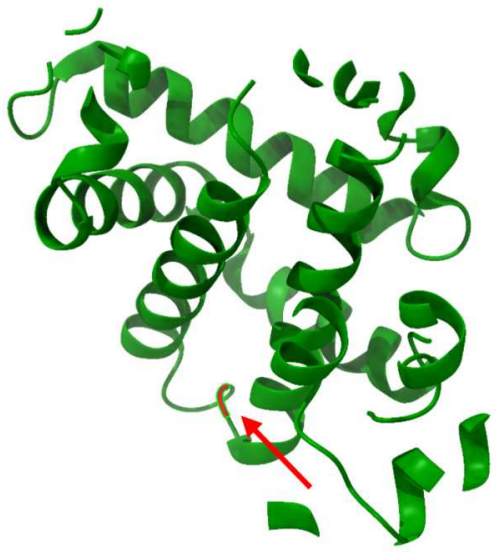

1c7d/A:117@N

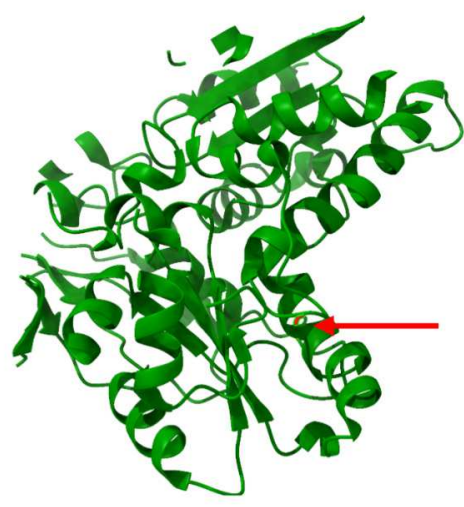

1hfe/L:246@N

FIG. S15. Visualization of the top nine activating neighborhoods of atomic filter (b) shown in Fig.3 The top-activating atom is the backbone nitrogen of the residue shown in red (zoom-in for clarity). Each of the top-activating nitrogens is located at a contact zone between two helical fragments. 


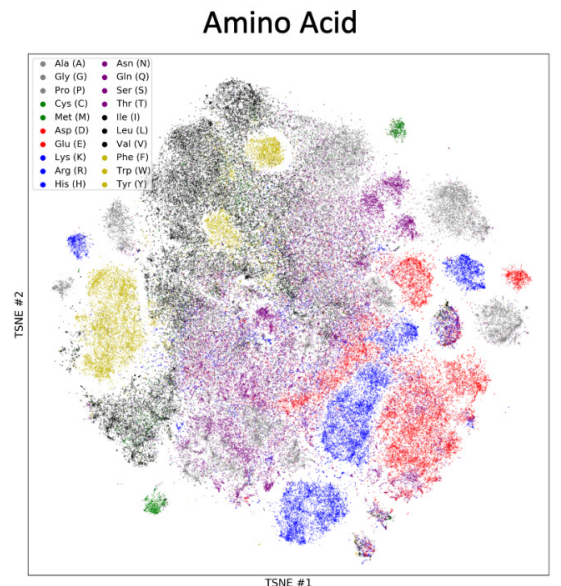

(a)

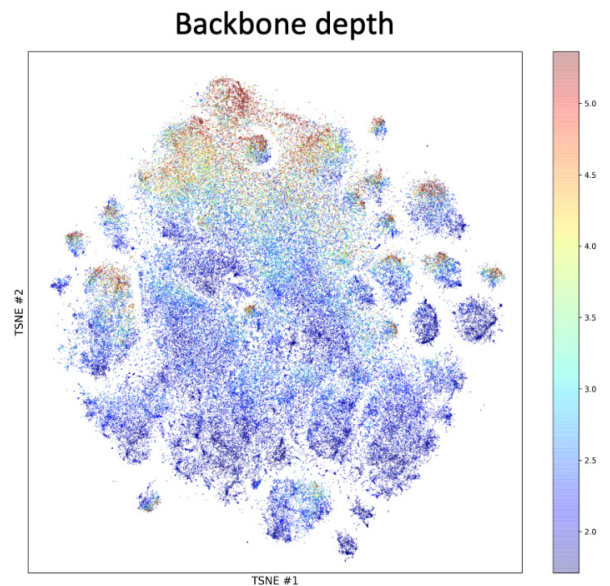

(c)

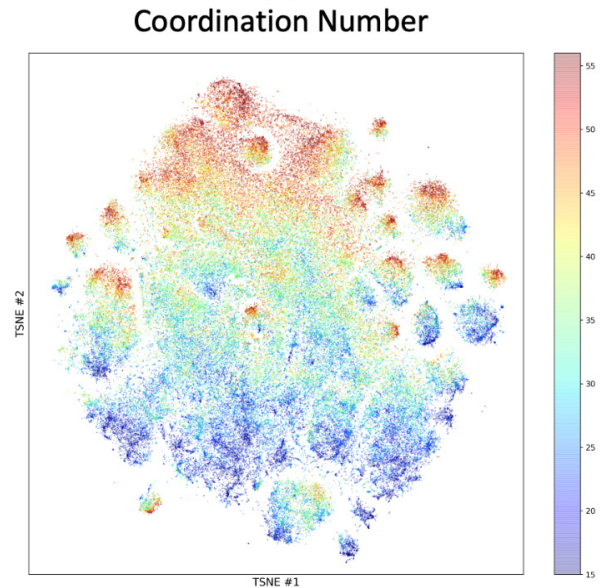

(e)

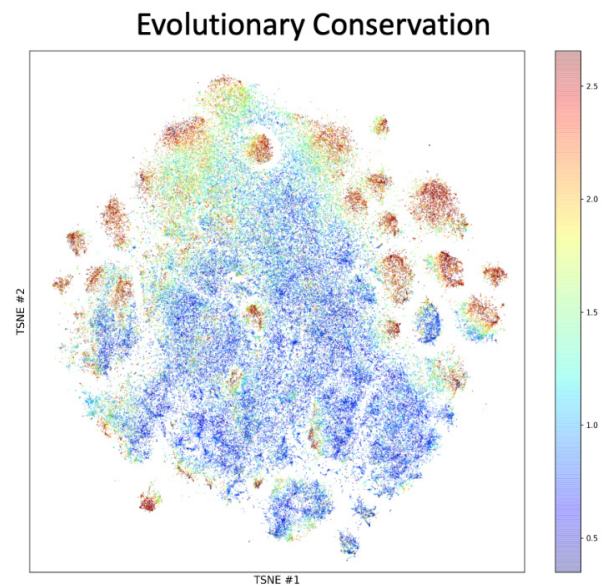

(b)

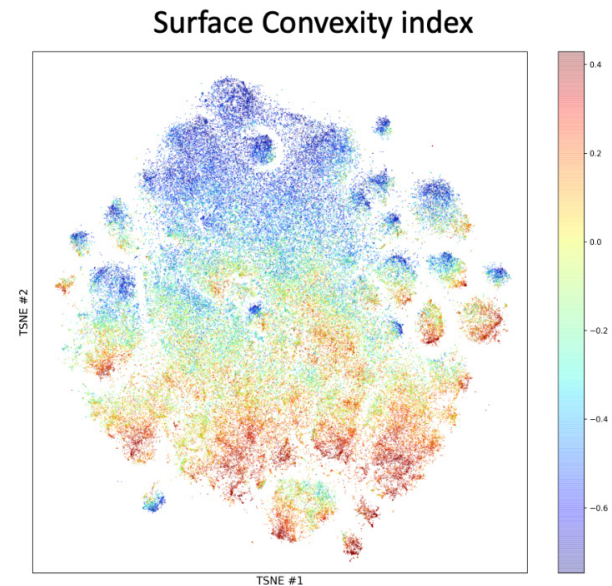

(d)

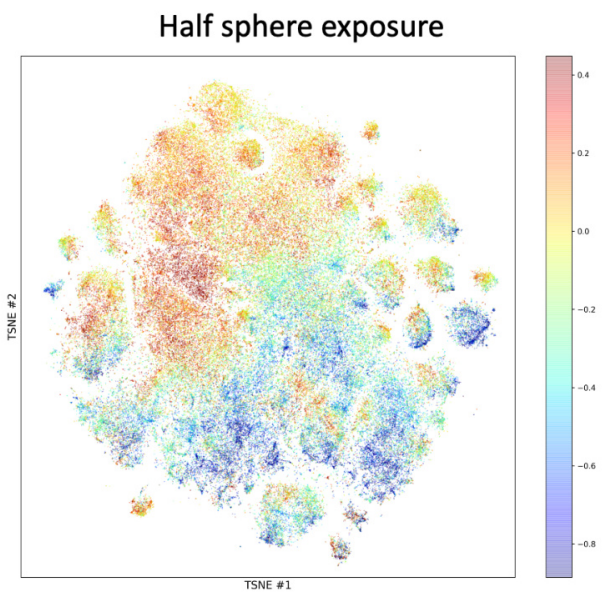

(f)

FIG. S16. Two-dimensional projection of the learnt amino acid scale representation using T-SNE [59] . Each point corresponds to one amino acid of a representative set of proteins. Coloring based on (a) Amino acid type (b) evolutionary conservation (c) backbone depth (d) surface convexity index (e) coordination number (f) Half-sphere exposure 

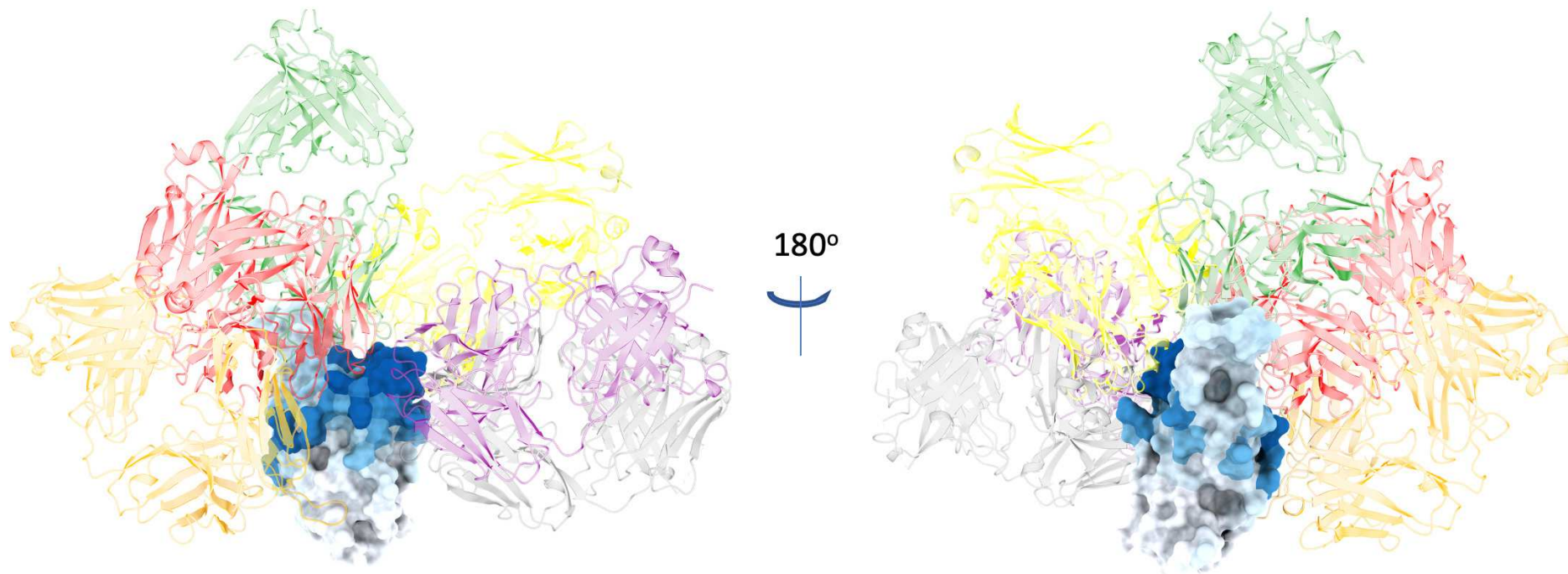

Receptor Binding Domain (a)
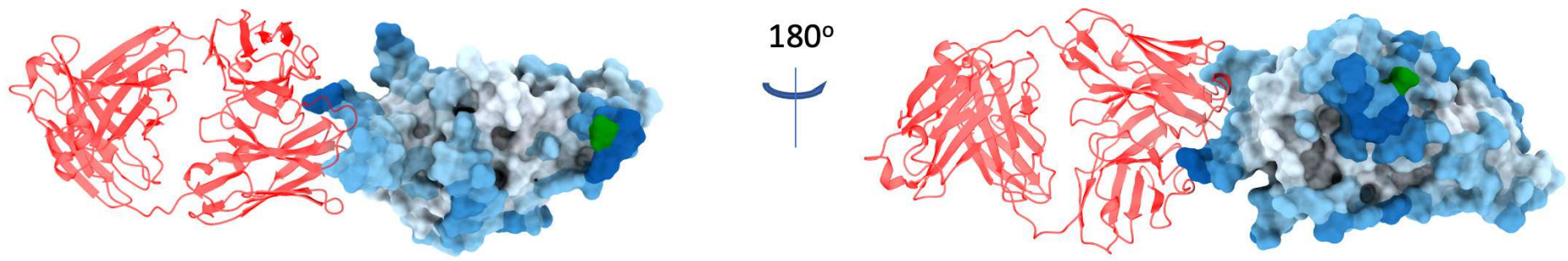

\section{$\mathrm{N}$-terminal Domain (b)}

FIG. S17. B-cell conformational epitope prediction on SARS-CoV2 Spike Protein a) Receptor Binding Domain (PDB ID 6xkp [90]) N-terminal domain (PDB ID 7l2c [94]). Domains depicted as surfaces, Green residues indicate glycosylated asparagines. 

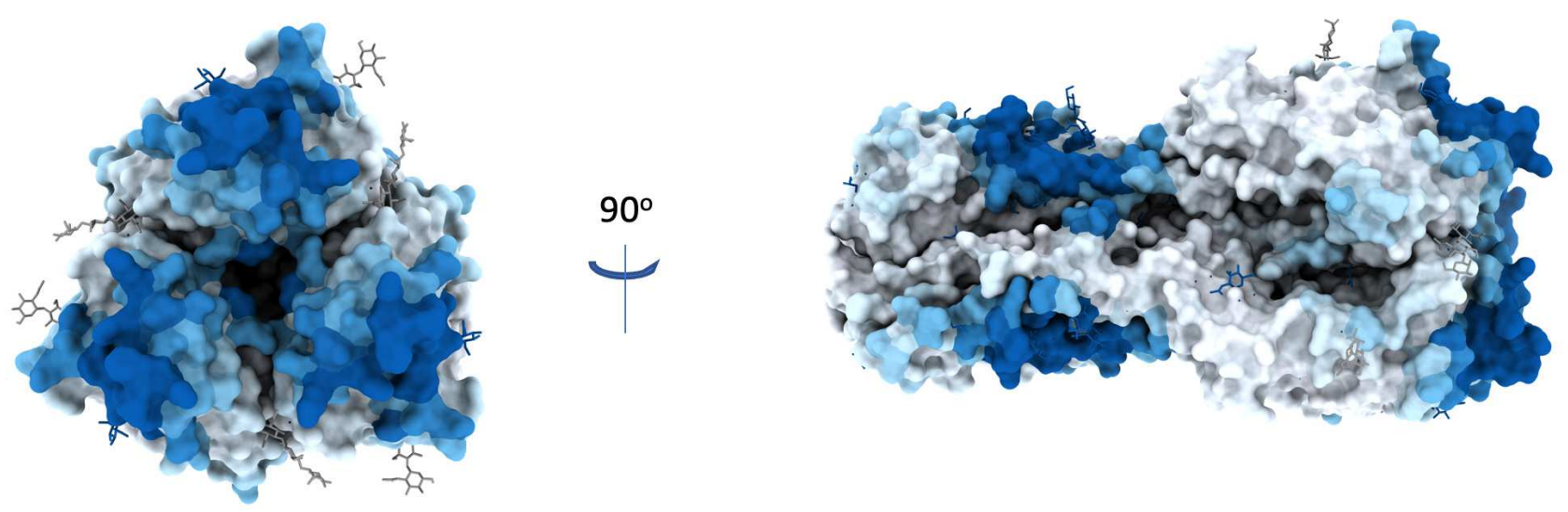

(a) Hemagglutinin Influenza H3
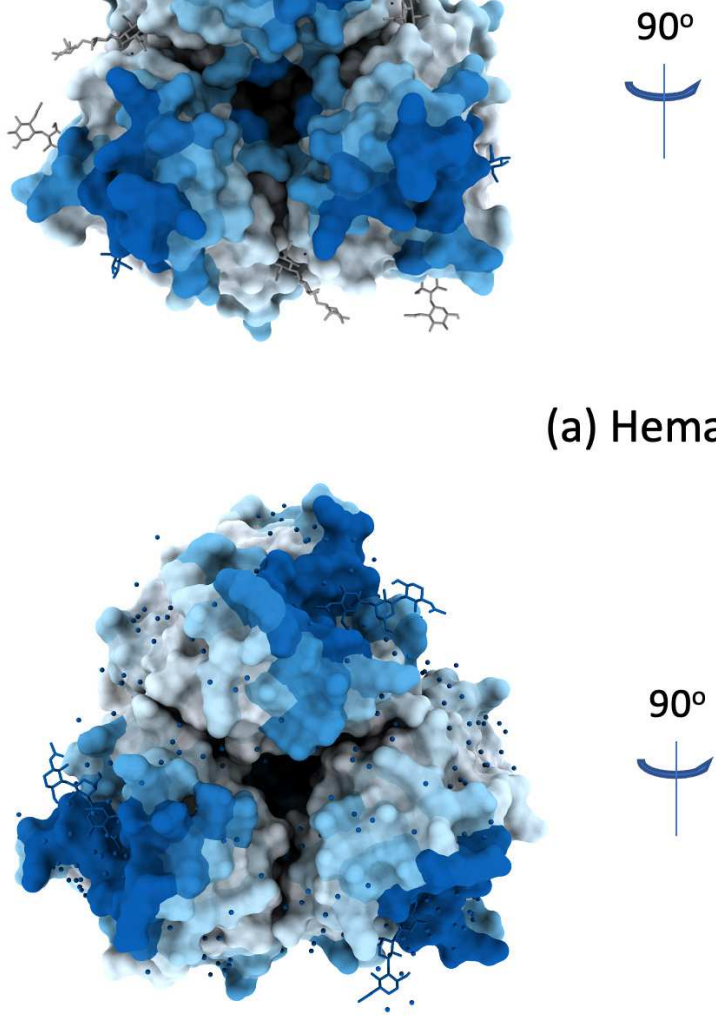

$90^{\circ}$
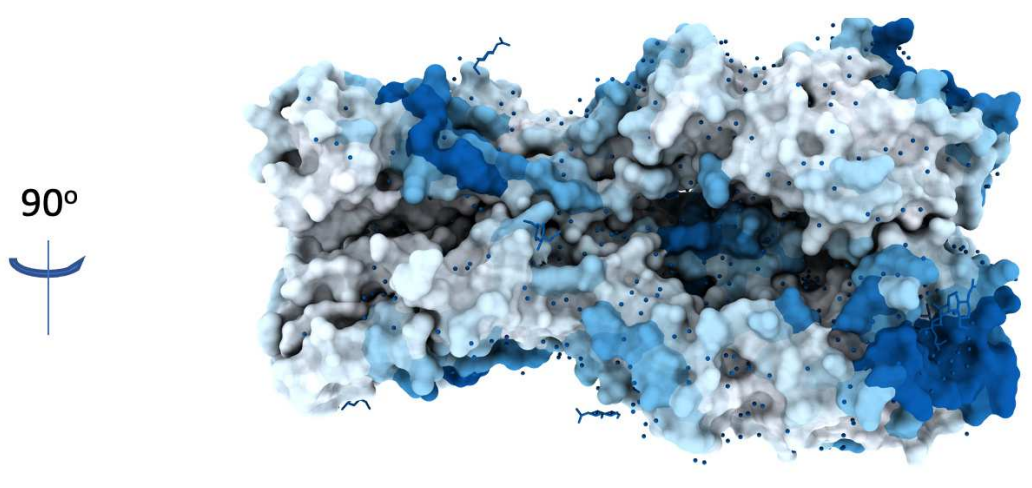

(b) Hemagglutinin Influenza H1
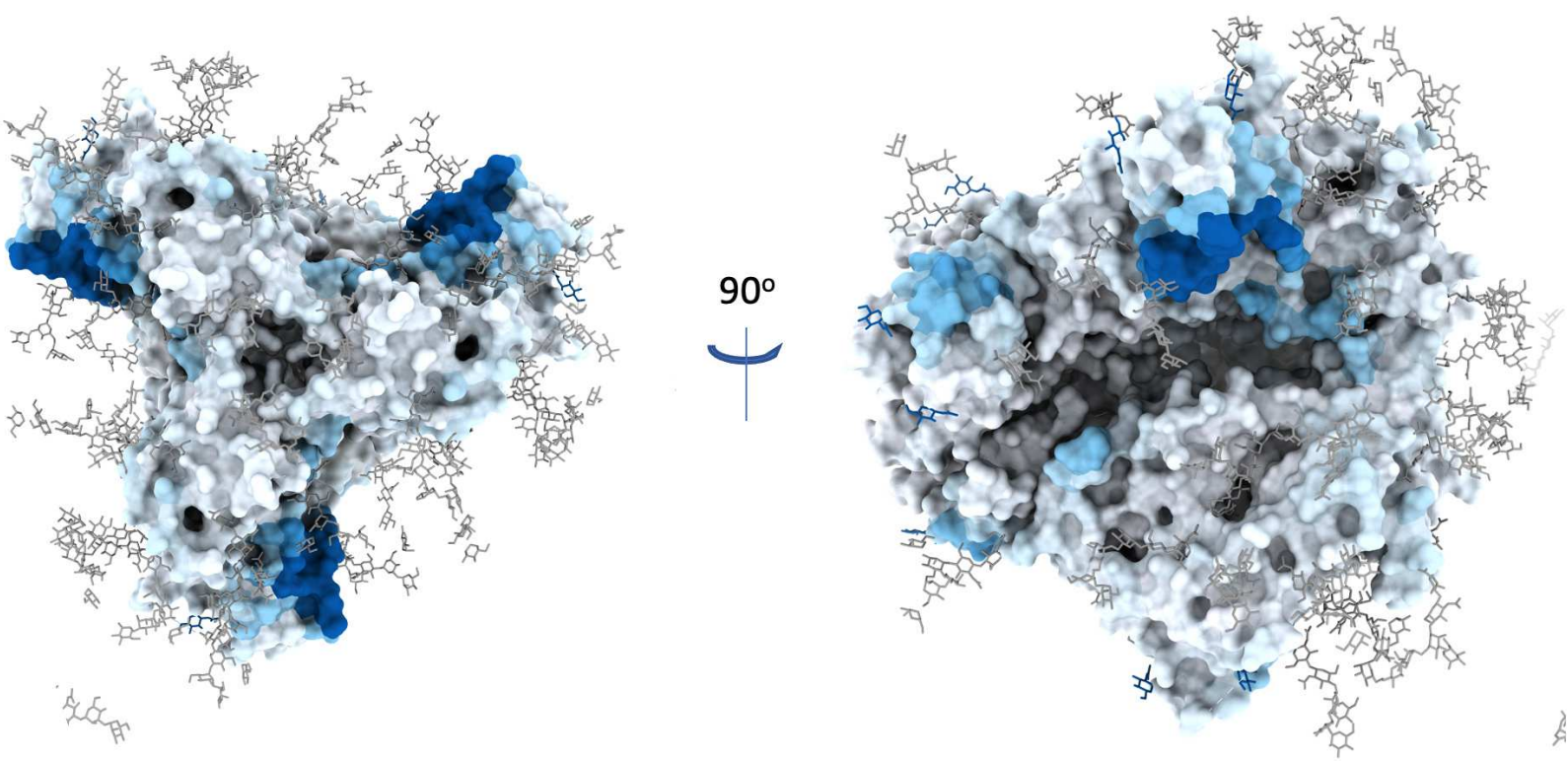

(c) HIV envelope protein

FIG. S18. Additional B-cell conformational epitope predictions with ScanNet a) Hemagglutinin trimer of Influenza H3 (PDB ID: 4o5n [95] ) b) Hemagglutinin trimer of Influenza H1 (PDB ID: 1rvx [96]) c) HIV Envelope protein (PDB ID 5fyl [97]). Predictions are performed in cross-validation setting (for each protein, we use the network that was not trained on it). Examples selected from [98] 
684 [13] R. Esmaielbeiki, K. Krawczyk, B. Knapp, J.-C. Nebel, and C. M. Deane, Progress and challenges in predicting protein 685 interfaces, Briefings in bioinformatics 17, 117 (2016).

686 [14] H. Neuvirth, R. Raz, and G. Schreiber, Promate: a structure based prediction program to identify the location of protein${ }_{687}$ protein binding sites, Journal of molecular biology 338, 181 (2004).

688 [15] J.-L. Chung, W. Wang, and P. E. Bourne, Exploiting sequence and structure homologs to identify protein-protein binding 689 sites, Proteins: Structure, Function, and Bioinformatics 62, 630 (2006).

${ }_{690}[16]$ A. Porollo and J. Meller, Prediction-based fingerprints of protein-protein interactions, Proteins: Structure, Function, and $691 \quad$ Bioinformatics 66, 630 (2007).

${ }_{692}[17]$ M. J. Sweredoski and P. Baldi, Pepito: improved discontinuous b-cell epitope prediction using multiple distance thresholds ${ }_{693}$ and half sphere exposure, Bioinformatics 24, 1459 (2008).

694 [18] S. K. Mishra, G. Kandoi, and R. L. Jernigan, Coupling dynamics and evolutionary information with structure to identify ${ }_{695}$ protein regulatory and functional binding sites, Proteins: Structure, Function, and Bioinformatics 87, 850 (2019).

696 [19] A. Klug and D. Rhodes, 'zinc fingers': a novel protein motif for nucleic acid recognition, Trends in Biochemical Sciences $697 \quad$ 12, $464(1987)$

698 [20] A. A. Bogan and K. S. Thorn, Anatomy of hot spots in protein interfaces, Journal of molecular biology 280, 1 (1998).

699 [21] M. Wensien, F. R. von Pappenheim, L.-M. Funk, P. Kloskowski, U. Curth, U. Diederichsen, J. Uranga, J. Ye, P. Fang, 700 K.-T. Pan, et al., A lysine-cysteine redox switch with an nos bridge regulates enzyme function, Nature 593, 460 (2021).

$701[22]$ A. Elnaggar, M. Heinzinger, C. Dallago, G. Rihawi, Y. Wang, L. Jones, T. Gibbs, T. Feher, C. Angerer, M. Steinegger,

709 [25] J. Ingraham, V. K. Garg, R. Barzilay, and T. Jaakkola, Generative models for graph-based protein design, (2019).

710 [26] X. Jing and J. Xu, Fast and effective protein model refinement by deep graph neural networks, bioRxiv (2020).

711 [27] F. Baldassarre, D. Menéndez Hurtado, A. Elofsson, and H. Azizpour, Graphqa: protein model quality assessment using 712 graph convolutional networks, Bioinformatics 37, 360 (2021).

713 [28] I. Wallach, M. Dzamba, and A. Heifets, Atomnet: a deep convolutional neural network for bioactivity prediction in $714 \quad$ structure-based drug discovery, arXiv preprint arXiv:1510.02855 (2015).

715 [29] M. Ragoza, J. Hochuli, E. Idrobo, J. Sunseri, and D. R. Koes, Protein-ligand scoring with convolutional neural networks, $716 \quad$ Journal of chemical information and modeling $\mathbf{5 7}, 942$ (2017).

717 [30] G. Pagès, B. Charmettant, and S. Grudinin, Protein model quality assessment using 3d oriented convolutional neural 718 networks, Bioinformatics 35, 3313 (2019).

${ }_{719}[31]$ R. Townshend, R. Bedi, P. Suriana, and R. Dror, End-to-end learning on 3d protein structure for interface prediction, 720 Advances in Neural Information Processing Systems 32, 15642 (2019).

721 [32] X. Wang, G. Terashi, C. W. Christoffer, M. Zhu, and D. Kihara, Protein docking model evaluation by 3d deep convolutional 
722 neural networks, Bioinformatics 36, 2113 (2020).

723 [33] I. Igashov, K. Olechnovic, M. Kadukova, Č. Venclovas, and S. Grudinin, Vorocnn: Deep convolutional neural network built ${ }_{724}$ on $3 \mathrm{~d}$ voronoi tessellation of protein structures, bioRxiv (2020).

725 [34] N. Renaud, C. Geng, S. Georgievska, F. Ambrosetti, L. Ridder, D. F. Marzella, A. M. Bonvin, and L. C. Xue, Deeprank: 726 A deep learning framework for data mining 3d protein-protein interfaces, Biorxiv (2021).

727 [35] S. Eismann, P. Suriana, B. Jing, R. J. Townshend, and R. O. Dror, Protein model quality assessment using rotation728 equivariant, hierarchical neural networks, arXiv preprint arXiv:2011.13557 (2020).

729 [36] P. Gainza, F. Sverrisson, F. Monti, E. Rodola, D. Boscaini, M. Bronstein, and B. Correia, Deciphering interaction finger730 prints from protein molecular surfaces using geometric deep learning, Nature Methods 17, 184 (2020).

731 [37] F. Sverrisson, J. Feydy, B. E. Correia, and M. M. Bronstein, Fast end-to-end learning on protein surfaces, in Proceedings 732 of the IEEE/CVF Conference on Computer Vision and Pattern Recognition (2021) pp. 15272-15281.

733 [38] M. M. Bronstein, J. Bruna, Y. LeCun, A. Szlam, and P. Vandergheynst, Geometric deep learning: going beyond euclidean 734 data, IEEE Signal Processing Magazine 34, 18 (2017).

735 [39] M. M. Bronstein, J. Bruna, T. Cohen, and P. Veličković, Geometric deep learning: Grids, groups, graphs, geodesics, and 736 gauges, arXiv preprint arXiv:2104.13478 (2021).

737 [40] J. Gilmer, S. S. Schoenholz, P. F. Riley, O. Vinyals, and G. E. Dahl, Neural message passing for quantum chemistry, in $738 \quad$ International conference on machine learning (PMLR, 2017) pp. 1263-1272.

739 [41] P. Veličković, G. Cucurull, A. Casanova, A. Romero, P. Lio, and Y. Bengio, Graph attention networks, arXiv preprint $740 \quad$ arXiv:1710.10903 (2017).

741 [42] O. Keskin, B. Ma, and R. Nussinov, Hot regions in protein-protein interactions: the organization and contribution of 742 structurally conserved hot spot residues, Journal of molecular biology 345, 1281 (2005).

743 [43] Y. Ofran and B. Rost, Protein-protein interaction hotspots carved into sequences, PLoS computational biology 3, e119 $744 \quad(2007)$.

745 [44] S. Dey, D. W. Ritchie, and E. D. Levy, Pdb-wide identification of biological assemblies from conserved quaternary structure 746 geometry, Nature methods 15, 67 (2018).

747 [45] P. J. Kundrotas, I. Anishchenko, T. Dauzhenka, I. Kotthoff, D. Mnevets, M. M. Copeland, and I. A. Vakser, Dockground: 748 a comprehensive data resource for modeling of protein complexes, Protein Science 27, 172 (2018).

749 [46] T. Chen and C. Guestrin, Xgboost: A scalable tree boosting system, in Proceedings of the 22nd acm sigkdd international $750 \quad$ conference on knowledge discovery and data mining (2016) pp. 785-794.

751 [47] M. Shatsky, R. Nussinov, and H. J. Wolfson, Multiprot - a multiple protein structural alignment algorithm, in International 752 Workshop on Algorithms in Bioinformatics (Springer, 2002) pp. 235-250.

753 [48] I. Sillitoe, N. Bordin, N. Dawson, V. P. Waman, P. Ashford, H. M. Scholes, C. S. Pang, L. Woodridge, C. Rauer, N. Sen, ${ }_{754}$ et al., Cath: increased structural coverage of functional space, Nucleic acids research 49, D266 (2021).

755 [49] E. Jurrus, D. Engel, K. Star, K. Monson, J. Brandi, L. E. Felberg, D. H. Brookes, L. Wilson, J. Chen, K. Liles, et al., 756 Improvements to the apbs biomolecular solvation software suite, Protein Science 27, 112 (2018).

757 [50] J. Dunbar, K. Krawczyk, J. Leem, T. Baker, A. Fuchs, G. Georges, J. Shi, and C. M. Deane, Sabdab: the structural $758 \quad$ antibody database, Nucleic acids research 42, D1140 (2014).

759 [51] J. V. Kringelum, C. Lundegaard, O. Lund, and M. Nielsen, Reliable b cell epitope predictions: impacts of method 760 development and improved benchmarking, PLoS Comput Biol 8, e1002829 (2012).

761 [52] M. Yuan, D. Huang, C.-C. D. Lee, N. C. Wu, A. M. Jackson, X. Zhu, H. Liu, L. Peng, M. J. van Gils, R. W. Sanders, 762 et al., Structural and functional ramifications of antigenic drift in recent sars-cov-2 variants, Science (2021).

763 [53] E. Shrock, E. Fujimura, T. Kula, R. T. Timms, I.-H. Lee, Y. Leng, M. L. Robinson, B. M. Sie, M. Z. Li, Y. Chen, et al., ${ }_{764}$ Viral epitope profiling of covid-19 patients reveals cross-reactivity and correlates of severity, Science $\mathbf{3 7 0}$ (2020).

765 [54] M. M. Sauer, M. A. Tortorici, Y.-J. Park, A. C. Walls, L. Homad, O. J. Acton, J. E. Bowen, C. Wang, X. Xiong, ${ }_{766}$ W. de van der Schueren, et al., Structural basis for broad coronavirus neutralization, Nature Structural \& Molecular 767 Biology , 1 (2021).

768 [55] Y. Watanabe, J. D. Allen, D. Wrapp, J. S. McLellan, and M. Crispin, Site-specific glycan analysis of the sars-cov-2 spike, $769 \quad$ Science 369, 330 (2020).

770 [56] A. M. Buckle, G. Schreiber, and A. R. Fersht, Protein-protein recognition: Crystal structural analysis of a barnase-barstar $771 \quad$ complex at 2.0-. ang. resolution, Biochemistry 33, 8878 (1994).

772 [57] G. Fenalti, R. H. Law, A. M. Buckle, C. Langendorf, K. Tuck, C. J. Rosado, N. G. Faux, K. Mahmood, C. S. Hampe, J. P. 773 Banga, et al., Gaba production by glutamic acid decarboxylase is regulated by a dynamic catalytic loop, Nature structural $774 \quad \&$ molecular biology 14, 280 (2007).

775 [58] T. D. Goddard, C. C. Huang, E. C. Meng, E. F. Pettersen, G. S. Couch, J. H. Morris, and T. E. Ferrin, Ucsf chimerax: $776 \quad$ Meeting modern challenges in visualization and analysis, Protein Science 27, 14 (2018).

777 [59] L. Van der Maaten and G. Hinton, Visualizing data using t-sne., Journal of machine learning research 9 (2008).

778 [60] W. Kabsch and C. Sander, Dictionary of protein secondary structure: pattern recognition of hydrogen-bonded and geo779 metrical features, Biopolymers: Original Research on Biomolecules 22, 2577 (1983).

780 [61] R. Amaro and A. Mulholland, Biomolecular simulations in the time of covid19, and after, Computing in Science \& 781 Engineering (2020).

782 [62] P. J. Cock, T. Antao, J. T. Chang, B. A. Chapman, C. J. Cox, A. Dalke, I. Friedberg, T. Hamelryck, F. Kauff, B. Wilczynski, 783 et al., Biopython: freely available python tools for computational molecular biology and bioinformatics, Bioinformatics 25, $784 \quad 1422(2009)$.

785 [63] If both are equally far away e.g. for isoleucine, we choose the first one according to the residue id. 
786 [64] M. Remmert, A. Biegert, A. Hauser, and J. Söding, Hhblits: lightning-fast iterative protein sequence searching by hmm787 hmm alignment, Nature methods 9, 173 (2012).

788 [65] M. Mirdita, L. von den Driesch, C. Galiez, M. J. Martin, J. Söding, and M. Steinegger, Uniclust databases of clustered 789 and deeply annotated protein sequences and alignments, Nucleic acids research 45, D170 (2017).

790 [66] S. Cocco, C. Feinauer, M. Figliuzzi, R. Monasson, and M. Weigt, Inverse statistical physics of protein sequences: a key 791 issues review, Reports on Progress in Physics 81, 032601 (2018).

792 [67] L. Posani, Inference and modeling of biological networks: a statistical-physics approach to neural attractors and protein 793 fitness landscapes, Ph.D. thesis, Université Paris sciences et lettres (2018).

794 [68] W. Chen, X. Han, G. Li, C. Chen, J. Xing, Y. Zhao, and H. Li, Deep rbfnet: Point cloud feature learning using radial 795 basis functions, arXiv preprint arXiv:1812.04302 (2018).

796 [69] C. R. Qi, H. Su, K. Mo, and L. J. Guibas, Pointnet: Deep learning on point sets for 3d classification and segmentation, in 797 Proceedings of the IEEE conference on computer vision and pattern recognition (2017) pp. 652-660.

${ }_{798}$ [70] C. R. Qi, L. Yi, H. Su, and L. J. Guibas, Pointnet++: Deep hierarchical feature learning on point sets in a metric space, 799 arXiv preprint arXiv:1706.02413 (2017).

800 [71] I. Igashov, N. Pavlichenko, and S. Grudinin, Spherical convolutions on molecular graphs for protein model quality assess801 ment, Machine Learning: Science and Technology (2021).

802 [72] J. Tubiana, S. Cocco, and R. Monasson, Learning protein constitutive motifs from sequence data, Elife 8, e39397 (2019).

803 [73] S. Ioffe and C. Szegedy, Batch normalization: Accelerating deep network training by reducing internal covariate shift, in $804 \quad$ International conference on machine learning (PMLR, 2015) pp. 448-456.

${ }_{805}$ [74] F. Pedregosa, G. Varoquaux, A. Gramfort, V. Michel, B. Thirion, O. Grisel, M. Blondel, P. Prettenhofer, R. Weiss, ${ }_{806}$ V. Dubourg, et al., Scikit-learn: Machine learning in python, the Journal of machine Learning research 12, 2825 (2011).

807 [75] J. Long, E. Shelhamer, and T. Darrell, Fully convolutional networks for semantic segmentation, in Proceedings of the IEEE $808 \quad$ conference on computer vision and pattern recognition (2015) pp. 3431-3440.

809 [76] D. P. Kingma and J. Ba, Adam: A method for stochastic optimization, arXiv preprint arXiv:1412.6980 (2014).

810 [77] M. Abadi, P. Barham, J. Chen, Z. Chen, A. Davis, J. Dean, M. Devin, S. Ghemawat, G. Irving, M. Isard, et al., Tensorflow:

811 A system for large-scale machine learning, in 12th \{USENIX\} symposium on operating systems design and implementation ${ }_{812} \quad(\{O S D I\} 16)(2016)$ pp. 265-283.

813 [78] F. Chollet, Deep learning with Python (Simon and Schuster, 2017).

814 [79] J. Song, H. Tan, K. Takemoto, and T. Akutsu, Hsepred: predict half-sphere exposure from protein sequences, Bioinformatics $815 \quad 24,1489$ (2008).

816 [80] S. Chakravarty and R. Varadarajan, Residue depth: a novel parameter for the analysis of protein structure and stability, $817 \quad$ Structure 7, 723 (1999).

${ }_{818}$ [81] M. F. Sanner, A. J. Olson, and J.-C. Spehner, Reduced surface: an efficient way to compute molecular surfaces, Biopolymers $819 \quad 38,305(1996)$.

820 [82] M. L. Connolly, Shape complementarity at the hemoglobin $\alpha 1 \beta 1$ subunit interface, Biopolymers 25, 1229 (1986).

${ }_{821}$ [83] L. Fu, B. Niu, Z. Zhu, S. Wu, and W. Li, Cd-hit: accelerated for clustering the next-generation sequencing data, Bioinfor822 matics 28, 3150 (2012).

${ }_{823}$ [84] W. Li and A. Godzik, Cd-hit: a fast program for clustering and comparing large sets of protein or nucleotide sequences, 824 Bioinformatics 22, 1658 (2006).

${ }_{825}$ [85] T. Nakamura, K. D. Yamada, K. Tomii, and K. Katoh, Parallelization of mafft for large-scale multiple sequence alignments, ${ }_{826}$ Bioinformatics 34, 2490 (2018).

827 [86] For the PPBS data set, $8.9 \%$ of the binding site residues are actually in unbound conformation, as their label was inferred ${ }_{828}$ from another pdb file, see data preparation.

829 [87] T. Kirys, A. M. Ruvinsky, D. Singla, A. V. Tuzikov, P. J. Kundrotas, and I. A. Vakser, Simulated unbound structures for ${ }_{830}$ benchmarking of protein docking in the dockground resource, BMC bioinformatics 16, 1 (2015).

831 [88] M. Yuan, H. Liu, N. C. Wu, C.-C. D. Lee, X. Zhu, F. Zhao, D. Huang, W. Yu, Y. Hua, H. Tien, et al., Structural basis of ${ }_{832}$ a public antibody response to sars-cov-2, BioRxiv (2020).

833 [89] N. C. Wu, M. Yuan, H. Liu, C.-C. D. Lee, X. Zhu, S. Bangaru, J. L. Torres, T. G. Caniels, P. J. Brouwer, M. J. Van Gils, ${ }_{834}$ et al., An alternative binding mode of ighv3-53 antibodies to the sars-cov-2 receptor binding domain, Cell reports 33, $835108274(2020)$.

836 [90] J. Kreye, S. M. Reincke, H.-C. Kornau, E. Sánchez-Sendin, V. M. Corman, H. Liu, M. Yuan, N. C. Wu, X. Zhu, C.-C. D. Lee, et al., A therapeutic non-self-reactive sars-cov-2 antibody protects from lung pathology in a covid-19 hamster model, Cell 183, 1058 (2020).

839 [91] J. Hansen, A. Baum, K. E. Pascal, V. Russo, S. Giordano, E. Wloga, B. O. Fulton, Y. Yan, K. Koon, K. Patel, et al., 840 Studies in humanized mice and convalescent humans yield a sars-cov-2 antibody cocktail, Science 369, 1010 (2020).

${ }_{841}[92]$ H. Liu, M. Yuan, D. Huang, S. Bangaru, F. Zhao, C.-C. D. Lee, L. Peng, S. Barman, X. Zhu, D. Nemazee, et al., A ${ }_{842}$ combination of cross-neutralizing antibodies synergizes to prevent sars-cov-2 and sars-cov pseudovirus infection, Cell host $843 \quad \&$ microbe 29, 806 (2021).

844 [93] H. Liu, N. C. Wu, M. Yuan, S. Bangaru, J. L. Torres, T. G. Caniels, J. Van Schooten, X. Zhu, C.-C. D. Lee, P. J. Brouwer, 845 et al., Cross-neutralization of a sars-cov-2 antibody to a functionally conserved site is mediated by avidity, Immunity $\mathbf{5 3}$, $8461272(2020)$.

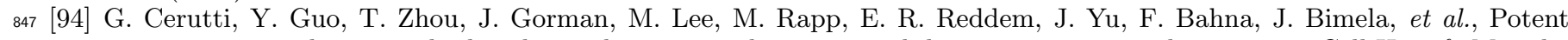
${ }_{848}$ sars-cov-2 neutralizing antibodies directed against spike n-terminal domain target a single supersite, Cell Host \& Microbe $849 \quad 29,819$ (2021). 
850 [95] P. S. Lee, N. Ohshima, R. L. Stanfield, W. Yu, Y. Iba, Y. Okuno, Y. Kurosawa, and I. A. Wilson, Receptor mimicry by 851 antibody f045-092 facilitates universal binding to the h3 subtype of influenza virus, Nature communications 5, 1 (2014).

852 [96] S. Gamblin, L. Haire, R. Russell, D. Stevens, B. Xiao, Y. Ha, N. Vasisht, D. Steinhauer, R. Daniels, A. Elliot, et al., The 853 structure and receptor binding properties of the 1918 influenza hemagglutinin, Science 303, 1838 (2004).

854 [97] G. B. Stewart-Jones, C. Soto, T. Lemmin, G.-Y. Chuang, A. Druz, R. Kong, P. V. Thomas, K. Wagh, T. Zhou, A.-J. ${ }_{855}$ Behrens, et al., Trimeric hiv-1-env structures define glycan shields from clades a, b, and g, Cell 165, 813 (2016).

856 [98] B. Hie, E. D. Zhong, B. Berger, and B. Bryson, Learning the language of viral evolution and escape, Science 371, 284 $857 \quad(2021)$. 


\section{Supplementary Files}

This is a list of supplementary files associated with this preprint. Click to download.

- TableArchitecture.pdf

- aafiltersScanNetBCE.pdf

- aafiltersScanNetPPBS.pdf

- atomfiltersScanNetPPBS.pdf 